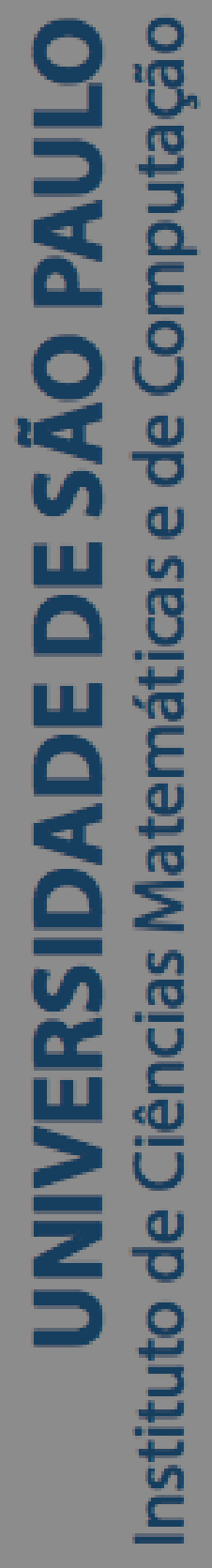

\title{
Regulador de Borel na K-teoria algébrica
}

\section{Piere Alexander Rodriguez Valerio}

Dissertação de Mestrado do Programa de Pós-Graduação em Matematica (PPG-Mat) 

Assinatura:

\title{
Piere Alexander Rodriguez Valerio
}

\section{Regulador de Borel na $K$-teoria algébrica}

\begin{abstract}
Dissertação apresentada ao Instituto de Ciências Matemáticas e de Computação - ICMC-USP, como parte dos requisitos para obtenção do título de Mestre em Ciências - Matemática. VERSÃO REVISADA
\end{abstract}

Área de Concentração: Matemática

Orientador: Prof. Dr. Behrooz Miirzai 
Ficha catalográfica elaborada pela Biblioteca Prof. Achille Bassi e Seção Técnica de Informática, ICMC/USP, com os dados inseridos pelo(a) autor(a)

Rodriguez Valerio, Piere Alexander Regulador de Borel na $K$-teoria algébrica / Piere Alexander Rodriguez Valerio; orientador Berhooz Miirzai. -- São Carlos, 2019. $97 \mathrm{p}$.

Dissertação (Mestrado - Programa de Pós-Graduação em Matemática) -- Instituto de Ciências Matemáticas e de Computação, Universidade de São Paulo, 2019.

1. K -grupos. 2. K -teoria algebraica. 3. Anel de inteiros. 4. Mapa regulador de Borel. I. Miirzai, Berhooz, orient. II. Título.

Bibliotecários responsáveis pela estrutura de catalogação da publicação de acordo com a AACR2: Gláucia Maria Saia Cristianini - CRB - 8/4938

Juliana de Souza Moraes - CRB - 8/6176 


\section{Piere Alexander Rodriguez Valerio}

\section{Borel Regulator in Aalgebraic K-theory}

Master dissertation submitted to the Instituto de Ciências Matemáticas e de Computação - ICMCUSP, in partial fulfillment of the requirements for the degree of the Master Program in Mathematics. FINAL VERSION

Concentration Area: Mathematics

Advisor: Prof. Dr. Behrooz Miirzai

USP - São Carlos

January 2019 

Este trabalho é dedicado às crianças adultas que, quando pequenas, sonharam em se tornar cientistas. 

Agradeço primeiramente à minha família, em especial a meus pais, Ciro Rodriguez e Carmen Valerio, e a meus irmãos Ciro J. Rodriguez, Danery Rodriguez e Emanoel M. dos Santos Freire, pelo carinho e apoio incondicional.

Agradeço também ao meu orientador, o Prof. Dr. Behrooz Miirzai,quem foi como um pai para mim, pelo tempo, paciência e apoio em tudo momento do mestrado.

Finalmente gostaria de agradecer ao ICMC pela oportunidade de fazer o mestrado e à CAPES pelo apoio financeiro. 

"Cualquiera puede ser un héroe, incluso un hombre que hace algo tan sencillo y reconfortante como ponerle un abrigo en los hombros a un niño para hacerle saber que la vida sigue."

(Bruce Wayne) 



\section{RESUMO}

RODRIGUEZ, P. A. Regulador de Borel na $K$-teoria algébrica. 2019. 97 p. Dissertação (Mestrado em Ciências - Matemática) - Instituto de Ciências Matemáticas e de Computação, Universidade de São Paulo, São Carlos - SP, 2019.

Neste trabalho, nos apresentamos a $K$-teoria algébrica a qual é um ramo da álgebra que associa para cada anel comutativo com unidade $R$, uma sequencia de grupos abelianos ditos de $n$-ésimos $K$-grupos do anel $R$, denotada por $K_{n}(R)$. A meados da década de 1950, Alexander Grothendieck da a definição do $K_{0}(R)$ de um anel $R$. Em 1962, Hyman Bass e Stephen Schanuel apresenta a primeira definição adequada do $K_{1}(R)$ de um anel $R$. Em 1970, Daniel Quillen da uma definição geral dos $K$-grupos de um anel $R$ a partir da +-construção do espaço classificante $B G L(R)$. Nosso interesse é o estudo dos $K$-grupos sobre o anel de inteiros $\mathscr{O}_{F}$ sobre um corpo numérico $F$. Usando alguns resultados de homologia dos grupos lineares, neste trabalho daremos a definição do mapa regulador de Borel.

Palavras-chave: $K$-grupos, $K$-teoria algebraica, Anel de inteiros, Mapa regulador de Borel. 



\section{ABSTRACT}

RODRIGUEZ, P. A. Borel Regulator in Aalgebraic K-theory. 2019. 97 p. Dissertação (Mestrado em Ciências - Matemática) - Instituto de Ciências Matemáticas e de Computação, Universidade de São Paulo, São Carlos - SP, 2019.

In this paper, we present the algebraic $K$-theory, which is a branch of algebra that associates to any ring with unit $R$ a sequence of abelian groups called $n$-th $K$-groups of $R$, denoted by $K_{n}(R)$. The mid-1950s, Alexander Grothendieck gave a definition of the $K_{0}(R)$ of any ring $R$. In 1962, Hyman Bass and Stephen Schanuel gave the first adequate definition of $K_{1}$ of any ring $R$. In 1970, Daniel Quillen gave a general definition of $K$-groups of any ring $R$ using the +-construction of the classifying space $B G L(R)$. Our interest is the study of the $K$-groups on the ring of integers $\mathscr{O}_{F}$ over a number field $F$. Using some results of homology of linear groups, this work will give the definition of Borel's regulator map.

Keywords: $K$-groups, Algebraic $K$-theory, Ring of integers, Borel's regulator map. 



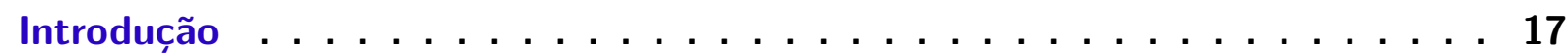

1 ÁlgEBRAS DE HOPF $\ldots \ldots \ldots \ldots \ldots$

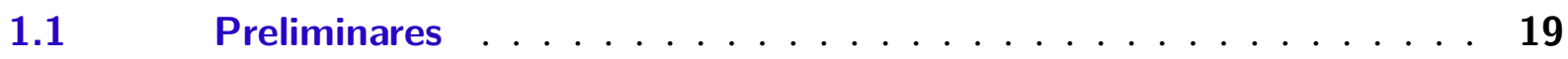

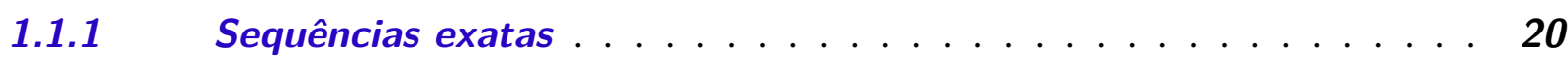

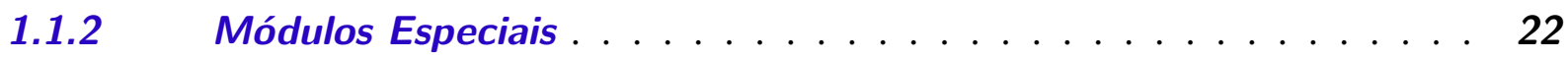

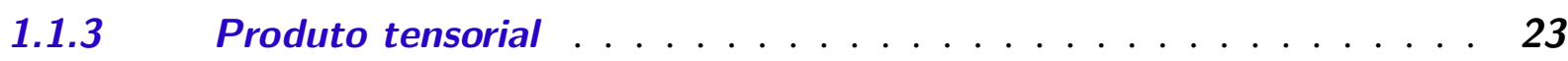

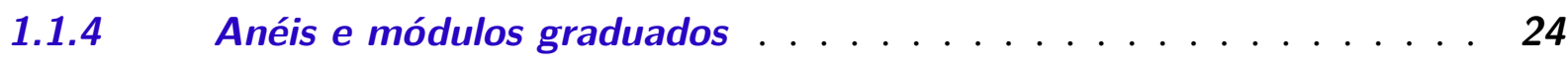

$1.2 \quad$ Álgebras e coálgebras graduadas . . . . . . . . . . . . 25

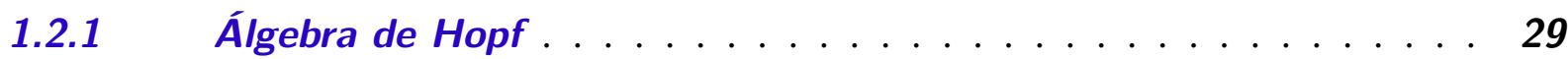

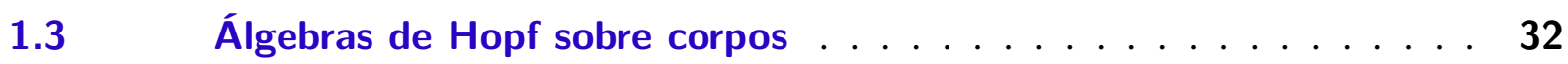

$1.4 \quad$ Álgebras de Lie e álgebras de Hopf $\ldots \ldots \ldots \ldots$

2 COHOMOLOGIA DE GRUPOS E O ISOMORFISMO DE VAN EST 37

2.1 Homologia e Cohomologia de um complexo . . . . . . . . 37

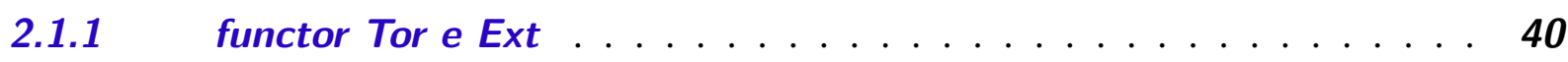

$2.2 \quad$ Homologia e Cohomologia de grupo . . . . . . . . . . . 41

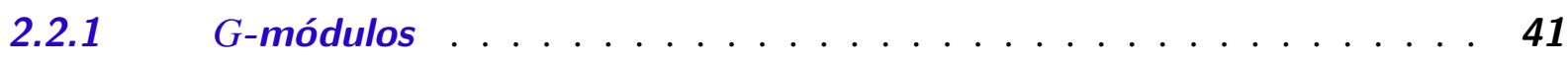

2.2.2 Homologia de grupo . . . . . . . . . . . . . . . . . 43

2.2.3 Cohomologia de grupo . . . . . . . . . . . . . . . . 44

2.3 Cohomologia de De Rham . . . . . . . . . . . . . . 46

$2.4 \quad$ Teorias de Homologia e Cohomologia . . . . . . . . . . . . 48

2.4.1 Homologia e Cohomologia singular . . . . . . . . . . . . . 48

2.4.2 Homotopia racional dos $\mathbf{H}$-espaços . . . . . . . . . . . . . . 49

2.4.2.1 Produto Whitehead . . . . . . . . . . . . . . . . . . . . 50

2.4.3 Relações entre a homologia e cohomologia de grupos . . . . . . 51

$2.5 \quad$ Cohomologia de Álgebra de Lie . . . . . . . . . . . . . . 52

2.5.1 Cohomologia de De Rham de um grupo de Lie . . . . . . . . . . 53

2.5.2 Grupos de Lie semisimples . . . . . . . . . . . . . . 56

2.5.3 Cohomologia relativa de álgebra de Lie . . . . . . . . . . . 59

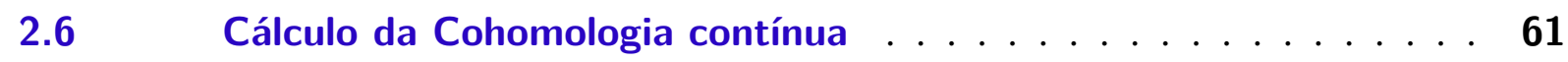

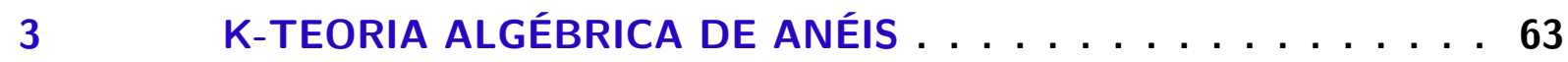

$3.1 \quad$ Grupo $K_{0}$ de um anel $\ldots \ldots \ldots \ldots$ 


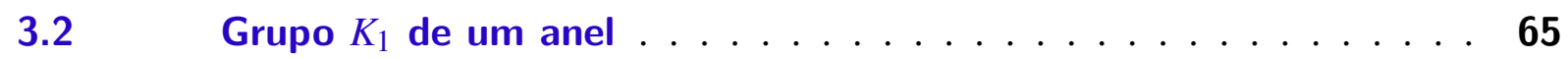

$3.3 \quad 0$ teorema da unidade $\ldots \ldots \ldots \ldots \ldots$. . . . . . . 68

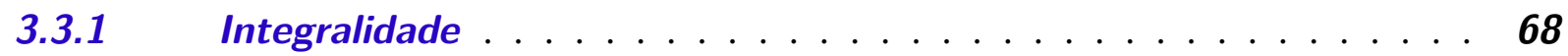

3.3.2 Retículo . . . . . . . . . . . . . . . . . . 71

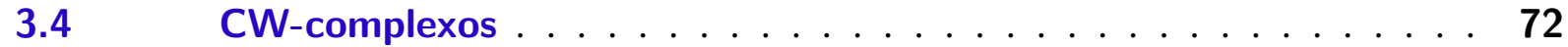

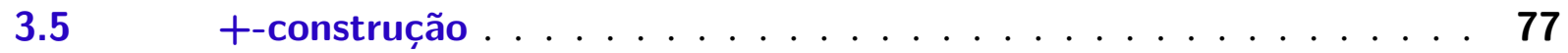

$3.6 \quad$ K-teoria de Quillen . . . . . . . . . . . . . . . . . . . 80

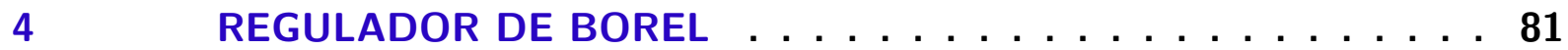

4.1 Definição do Regulador de Borel . . . . . . . . . . . . . . 81

$4.2 \quad \mathrm{O}$ posto do grupo $K_{m}\left(\mathscr{O}_{F}\right) \ldots \ldots \ldots \ldots$

4.3 Os valores da Função Zeta de Dedekind . . . . . . . . . . . 85

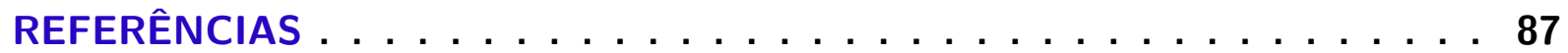

APÊNDICE A GRUPOS ALGEBRICOS . . . . . . . 89

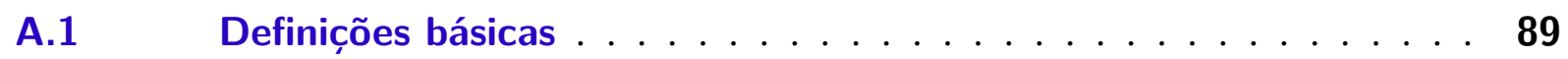

A.2 Extensão e restrição de escalares . . . . . . . . . . . 90

APÊNDICE B TEORIA DE HOMOTOPIA . . . . . . 93

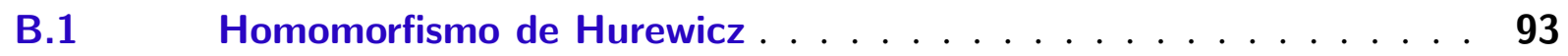

B.2 Teorema dos coeficientes universais . . . . . . . . . . 93

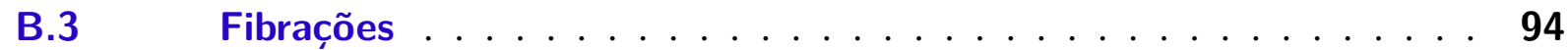

B.4 Sequência espectral de Serre-Leray $\ldots \ldots \ldots \ldots \ldots$ 
O objetivo deste trabalho é dar a construção do mapa regular de Borel (Definição 67). Iniciamos definindo o mapa regulador de Dirichlet. Seja $F$ um corpo numérico, seja $\mathscr{O}_{F}$ o anel de inteiros e seja $\mathscr{O}_{F}^{\times}$o grupo das unidades de $\mathscr{O}_{F}$. Seja $r_{1}$ (respectivamente $2 r_{2}$ ) o numero de mergulhos reais (respectivamente complexos) de $F$. No estudo de $\mathscr{O}_{F}^{*}$, Dirichlet introduze o mapa

$$
\rho: \mathscr{O}_{F}^{\times} \longrightarrow \mathbb{R}^{r_{1}+r_{2}} .
$$

A imagem deste mapa esta contida num hiperplano $H$. Mais ainda, $\rho\left(\mathscr{O}_{F}^{\times}\right)$é um retículo de $H$. Isto é

$$
\rho \otimes \mathbb{R}: \mathscr{O}_{F}^{\times} \otimes \mathbb{R} \longrightarrow H .
$$

é um isomorfismo. Em particular o posto de $\mathscr{O}_{F}^{\times}$é $r_{1}+r_{2}-1$. Seja $R_{D}=\operatorname{Vol}\left(H / \rho\left(\mathscr{O}_{F}^{\times}\right)\right)$o covolume do retículo. Este numero é chamado regulador de Dirichlet. $\mathrm{O}$ fato mais interessante sobre este regulador é a formula de numero de classe:

$$
R_{D}=\frac{\omega}{h_{F}} \lim _{s \longrightarrow 0} \zeta_{F}(s) s^{-\left(r_{1}+r_{2}-1\right)},
$$

onde $\zeta_{F}$ é a função zeta de Dedekind do corpo $F, \omega$ é o numero de raízes da unidade e $h_{F}$ é o numero de classe. Dado que a função zeta de Dedekind é definida usando dados locais nos ideais primos de $\mathscr{O}_{F}$, esta formula pode verse como um principio local ao global altamente não trivial. Para o fato de numero de classe veja-se (NEUKIRCH, 1937).

Recordando que $\mathscr{O}_{F}^{\times}$é o grupo $K_{1}\left(\mathscr{O}_{F}\right)$ (Teorema 18). A fim de generalizar a formula (1) aos $K$-grupos de ordem superior, Borel introduze , para todo $n \geq 2$, o morfismo

$$
r_{\mathrm{Bo}}^{\prime}: K_{2 n-1}\left(\mathscr{O}_{F}\right) \longrightarrow V_{n}
$$

onde $V_{n}$ é um espaço vetorial real de dimensão

$$
\operatorname{dim}_{\mathbb{R}}\left(V_{n}\right)=d_{n}=\left\{\begin{array}{llll}
r_{1}+r_{2} & \text {, se } & n \equiv 1 & \bmod 2 \\
r_{2} & \text {, se } & n \equiv 0 & \bmod 2 .
\end{array}\right.
$$

Esses morfismos são ditos de mapas reguladores de Borel. Mais ainda, Borel tem provado que $r_{\mathrm{Bo}}^{\prime}$ é um retículo de $V_{n}$ (Corolário 3). Como consequência obtemos o posto do grupo $K_{2 n-1}\left(\mathscr{O}_{F}\right)$ é $d_{n}$.

Lichtenbaum em (LICHTENBAUM, 1973) conjetura que, se escolhemos um natural retículo $L^{\prime}$ em $V_{n}$ e definimos

$$
R_{\mathrm{Bo}, n}^{\prime}=\operatorname{CoVol}\left(r_{\mathrm{Bo}}^{\prime}\left(K_{2 n-1}\left(\mathscr{O}_{F}\right)\right), L^{\prime}\right),
$$


então se verifica que

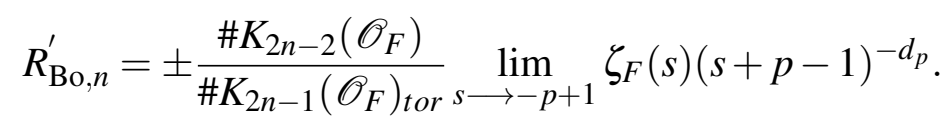

Liechtenbaum da uma concreta escolha do retículo $L^{\prime}$, mais ressaltou que, devido à falta de exemplos na época, pode ser necessário ajustar a fórmula por algum poder de $\pi$ e algum número racional.

Em (BOREL, 1977) Borel mostra que

$$
R_{\mathrm{Bo}, n}^{\prime} \sim \pi^{-d_{n}} \lim _{s \rightarrow-n+1} \zeta_{F}(s)(s+n-1)^{-d_{n}}
$$

onde $a \sim b$ significa que existe um elemento $q \in \mathbb{Q}^{\times}$tais que $q a=b$. O numero $R_{B o, n}^{\prime}$ é chamado o regulador de Borel.

Observação 1. O subíndice usado aqui não coincide com a convencional usada em (BOREL, 1977). Em particular o regulador de Borel $R_{\mathrm{Bo}, n}^{\prime}$ é $R_{n-1}$ com a notação de (BOREL, 1977). 
Neste capitulo daremos a definição de álgebras de Hopf, $H$-espaço e suas propriedades básicas. Denotamos por $\mathbb{N}:=\{0,1,2, \cdots\}$ o conjunto dos números naturais.

\subsection{Preliminares}

Um anel $R$ é um grupo abeliano $(R,+)$, munido de um produto associativo que é distributivo com respeito à adição.

Definição 1. Seja $R$ um anel comutativo com unidade. Um $R$-modulo $M$ é um grupo abeliano, escrito aditivamente, com um produto escalar, $R \times M \longrightarrow M$, escrito por $(r, m) \longmapsto r m$ verificando o seguinte:

(1) $r(m+n)=r m+r n$ e $(r+s) m=r m+s m$ (distributivo),

(2) $r(s m)=(r s) m($ associativo),

(3) $1 m=m$ (unitário).

Um submódulo $N$ de $M$ é um subgrupo que é fechado sob o produto, isto é, $r n \in N$ para todo $r \in R$ e $n \in N$.

Por exemplo, no caso de $R$ ser um corpo, um $R$-modulo é o mesmo que um $R$-espaço vetorial. Sempre entenderemos um anel $R$ como um anel comutativo com unidade.

Definição 2. Sejam $R$ um anel, $M, N R$-módulos. Dizemos que um mapa $\alpha: M \longrightarrow N$ é um $R$-homomorfismo se,

$$
\alpha(r m+s n)=r \alpha(m)+s \alpha(n),
$$

onde $m \in M, n \in N$ e $r, s \in R$. O conjunto dos $R$-homomorfismo $\alpha$ é denotado $\operatorname{por} \operatorname{Hom}_{R}(M, N)$ ou simplesmente por $\operatorname{Hom}(M, N)$. Um homomorfismo $\alpha$ é dito de isomorfismo se é bijetivo. 
Para cada homomorfismo $\alpha: M \longrightarrow N$ definimos seu núcleo e a sua imagem

$$
\operatorname{ker}(\alpha):=\alpha^{-1}(0) \subset M \text { e } \operatorname{Im}(\alpha):=\alpha(M) \subset N
$$

Eles são definidos como conjuntos, mais eles são submódulos.

Observação 2. Podemos dar uma estrutura de $R$-modulo a $\operatorname{Hom}_{R}(M, N)$ com adição e produto por um escalar dado por:

$$
(\alpha+\beta)(m):=\alpha(m)+\beta(m) \text { e }(r \alpha)(m):=r(\alpha(m))=\alpha(r m),
$$

onde $r \in R$ e $m \in M$. Dados os homomorfismos $\alpha: L \longrightarrow M$ e $\beta: N \longrightarrow P$ eles induz, via composição, um mapa

$$
\begin{aligned}
\operatorname{Hom}(\alpha, \beta): \operatorname{Hom}(M, N) & \longrightarrow \operatorname{Hom}(L, P) \\
f & \longmapsto \beta \circ f \circ \alpha,
\end{aligned}
$$

o qual é um homomorfismo. Quando $\alpha$ é o homomorfismo identidade $\mathrm{id}_{M}$, denotamos por $\beta_{*}$ ao homomorfismo $\operatorname{Hom}\left(\mathrm{id}_{M}, \beta\right)$; analogamente, denotamos por $\alpha^{*}$ ao homomorfismo $\operatorname{Hom}\left(\alpha, \mathrm{id}_{N}\right)$. Na lingua da Teoria de Categorias, podemos ver a $\operatorname{Hom}(M, \cdot)$ como um functor covariante e ao $\operatorname{Hom}(\cdot, N)$ como um functor contravariante definidos por

$$
\begin{aligned}
\operatorname{Hom}(M, \cdot): \operatorname{Mod}_{\mathrm{R}} & \longrightarrow \operatorname{Mod}_{\mathrm{R}} \\
N & \longmapsto \operatorname{Hom}(M, N) \\
\left(f: N \rightarrow N^{\prime}\right) & \longmapsto\left(f_{*}: \operatorname{Hom}(M, N) \rightarrow \operatorname{Hom}\left(M, N^{\prime}\right)\right),
\end{aligned}
$$

e

$$
\begin{aligned}
\operatorname{Hom}(\cdot, N): \operatorname{Mod}_{\mathrm{R}} & \longrightarrow \operatorname{Mod}_{\mathrm{R}} \\
M & \longmapsto \operatorname{Hom}(M, N) \\
\left(f: M \rightarrow M^{\prime}\right) & \longmapsto\left(f^{*}: \operatorname{Hom}\left(M^{\prime}, N\right) \rightarrow \operatorname{Hom}(M, N)\right),
\end{aligned}
$$

onde $\operatorname{Mod}_{\mathrm{R}}$ denota a categoria dos $R$-módulos.

\subsubsection{Sequências exatas}

Definição 3. Uma (finita ou infinita) sequência de $R$-homomorfismos

$$
\cdots \longrightarrow M_{i-1} \stackrel{\alpha_{i-1}}{\longrightarrow} M_{i} \stackrel{\alpha_{i}}{\longrightarrow} M_{i+1} \longrightarrow \cdots
$$

é dita exata em $M_{i}$ se $\operatorname{ker}\left(\alpha_{i}\right)=\operatorname{Im}\left(\alpha_{i-1}\right)$. A sequência é dita exata se é exata em cada $M_{i}$, excepto no inicio ou no final.

Exemplo 1. (1) Uma sequência $0 \longrightarrow L \stackrel{\alpha}{\longrightarrow} M$ é exata se e somente se $\alpha$ é injetiva. Neste caso, podemos identificar $L \operatorname{com} \alpha(L)$. Dualmente, a situação análoga com as setas invertidas, uma sequência $M \stackrel{\beta}{\longrightarrow} N \longrightarrow 0$ é exata se e somente se $\beta$ é sobrejetora. 
(2) Uma sequência $0 \longrightarrow L \stackrel{\alpha}{\longrightarrow} M \stackrel{\beta}{\longrightarrow} N \longrightarrow 0$ é exata se e somente se $\alpha$ é injetiva, $\beta$ é sobrejetora e $\operatorname{Im}(\alpha)=\operatorname{ker}(\beta)$. Neste caso a sequência é dita de sequência exata curta.

Definição 4. Dizemos que uma sequência exata curta

$$
0 \longrightarrow M^{\prime} \stackrel{\alpha}{\longrightarrow} M \stackrel{\beta}{\longrightarrow} M^{\prime \prime} \longrightarrow 0
$$

cinde se existe um isomorfismo $\varphi: M \stackrel{\sim}{\longrightarrow} M^{\prime} \oplus M^{\prime \prime}$ tais que $\pi_{M^{\prime}} \circ \varphi \circ \alpha=\mathrm{id}_{M^{\prime}}$ e $\beta=\pi_{M^{\prime \prime}} \circ$ $\varphi$ onde $\pi_{M^{\prime}}: M^{\prime} \oplus M^{\prime \prime} \longrightarrow M^{\prime}$ e $\pi_{M^{\prime \prime}}: M^{\prime} \oplus M^{\prime \prime} \longrightarrow M^{\prime \prime}$ são as projeções. Dizemos que um homomorfismo $\rho: M \longrightarrow M^{\prime}$ é uma retração de $\alpha$ se $\rho \circ \alpha=\mathrm{id}_{M^{\prime}}$. Dualmente, dizemos que um homomorfismo $\sigma: M^{\prime \prime} \longrightarrow M$ é uma secção de $\beta$ se $\beta \circ \sigma=\operatorname{id}_{M^{\prime \prime}}$

Proposição 1. Seja $0 \longrightarrow M^{\prime} \stackrel{\alpha}{\longrightarrow} M \stackrel{\beta}{\longrightarrow} M^{\prime \prime} \longrightarrow 0$ uma sequência exata curta. Então as seguintes condições são equivalentes:

(1) A sequência cinde.

(2) Existe uma retração $\rho: M \longrightarrow M^{\prime}$ de $\alpha$.

(3) Existe uma secção $\sigma: M^{\prime \prime} \longrightarrow M$ de $\beta$.

Demonstração. Vide [(ALTMAN; KLEIMAN, 2012), Proposição 5.9]

Lema 1 (Lema dos cinco). Consideremos o seguinte diagrama de $R$-módulos

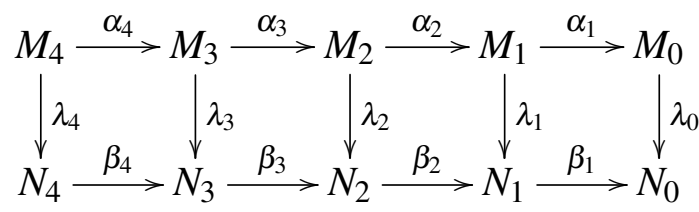

assumamos que as filas são exatas, então

(1) Se $\lambda_{3}$ e $\lambda_{1}$ são sobrejetoras e $\lambda_{0}$ é injetora, então $\lambda_{2}$ é sobrejetora.

(2) Se $\lambda_{3}$ e $\lambda_{1}$ são injetoras e $\lambda_{4}$ é sobrejetora, então $\lambda_{2}$ é injetora.

Demonstração. Vide [(ALTMAN; KLEIMAN, 2012), Exercício 5.14]

Observação 3. Segue do Lema dos cinco que, se $\lambda_{0}, \lambda_{1}, \lambda_{3}, \lambda_{4}$ são isomorfismos então $\lambda_{2}$ é um isomorfismo. 


\subsubsection{Módulos Especiais}

Agora daremos a definição de alguns de $R$-módulos especiais, os quais serão usados nas construções futuras.

Definição 5. Sejam $R$ um anel, $\Lambda$ um conjunto e $M$ um $R$-modulo. Dado um elemento $m_{\lambda} \in M$ por cada $\lambda \in \Lambda$, o submódulo gerado por $\left\{m_{\lambda}\right\}_{\lambda \in \Lambda}$, é o menor submódulo contendo a $\left\{m_{\lambda}\right\}_{\lambda \in \Lambda}$, isto é, é o conjunto das sumas finitas da forma $\sum r_{\lambda} m_{\lambda} \operatorname{com} r_{\lambda} \in R$, o qual é um submódulo.

Os elementos $\left\{m_{\lambda}\right\}_{\lambda \in \Lambda}$ são ditos de linearmente independente se, sempre que $\sum r_{\lambda} m_{\lambda}=0$ implica que $r_{\lambda}=0$ para todo $\lambda \in \Lambda$. Finalmente, dizemos que $\left\{m_{\lambda}\right\}_{\lambda \in \Lambda}$ formam uma base de $M$ se eles são linearmente independente e geram $M$.

Dizemos que $M$ é finitamente gerado se ele possui um numero finito de geradores. Dizemos que $M$ é livre se admite uma base. Pode-se provar que duas bases de um modulo livre $M$ finitamente gerado tem o mesmo numero de elementos, neste caso o numero de elementos de uma base é dito de posto do $M$.

Exemplo 2. Seja $R$ um anel, definimos o $R$-modulo $R^{\oplus \Lambda}$ dado por

$$
R^{\oplus \Lambda}=\left\{\left(r_{\lambda}\right): r_{\lambda} \in R \operatorname{com} r_{\lambda}=0 \text { para quase todo } \lambda \in \Lambda\right\}
$$

com a adição e produto escalar em cada componente. Ele tem uma base canônica, a qual consiste dos elementos $e_{\mu}$ cuja $\mu$-ésima componente toma o valor da função delta de Kronecker, isto é,

$$
e_{\mu}:=\left(\delta_{\mu \lambda}\right) \text { onde } \delta_{\mu \lambda}=\left\{\begin{array}{lll}
1 & \text { se } & \lambda=\mu \\
0 & \text { se } & \lambda \neq \mu
\end{array} .\right.
$$

Isto é o exemplo estândar de um $R$-modulo livre. Pode-se mostrar facilmente que um $R$-modulo livre é isomorfo a um modulo da forma $R^{\oplus \Lambda}$, onde $\Lambda$ é o conjunto de índice da base.

Definição 6. Uma apresentação livre de um $R$-modulo $M$ é uma sequência exata

$$
F_{1} \longrightarrow F_{2} \longrightarrow M \longrightarrow 0
$$

onde $F_{1}, F_{2}$ são $R$-módulos livres. Se $F_{1}, F_{2}$ são $R$-módulos de posto finito, então a apresentação é dita finita. Se $M$ tem apresentação finita, então $M$ é dito finitamente apresentado.

Definição 7. Um $R$-módulo $P$ é dito de projetivo se, dados quaisquer homomorfismo sobrejetor $\beta: M \longrightarrow N$, homomorfismo $\alpha: P \longrightarrow N$, existe um homomorfismo $\lambda: P \longrightarrow M$ tais que, $\alpha=\beta \circ \lambda$.

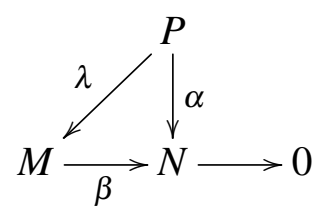


Na língua das categorias, um $R$-módulo $P$ é projetivo se o functor $\operatorname{Hom}(P, \cdot)$ é exato. No seguinte teorema damos caracterizações para que um $R$-modulo seja projetivo.

Teorema 1. Sejam $R$ um anel e $P$ um $R$-modulo, os seguintes enunciados são equivalentes:

(1) O modulo $P$ é projetivo.

(2) Toda sequência exata curta $0 \longrightarrow L \longrightarrow M \longrightarrow P \longrightarrow 0$ cinde.

(3) Existe um modulo $K$ tais que $K \oplus P$ é livre.

(4) Toda sequência exata $N^{\prime} \longrightarrow N \longrightarrow N^{\prime \prime}$ induz uma sequência exata

$$
\operatorname{Hom}\left(P, N^{\prime}\right) \longrightarrow \operatorname{Hom}(P, N) \longrightarrow \operatorname{Hom}\left(P, N^{\prime \prime}\right)
$$

(5) Todo homomorfismo sobrejetor $\beta: M \longrightarrow N$ induz um homomorfismo sobrejetor

$$
\beta_{*}: \operatorname{Hom}(P, M) \longrightarrow \operatorname{Hom}(P, N) \text {. }
$$

Demonstração. Vide [(ALTMAN; KLEIMAN, 2012), Proposição 5.22]

Segue do teorema anterior que todo $R$-módulo livre é projetivo.

Definição 8. Um $R$-módulo $Q$ é dito de injectivo se, dados quaisquer homomorfismo injetor $\beta: M \longrightarrow N$, homomorfismo $\alpha: M \longrightarrow Q$, existe um homomorfismo $\lambda: N \longrightarrow Q$ tais que, $\alpha=\lambda \circ \beta$.

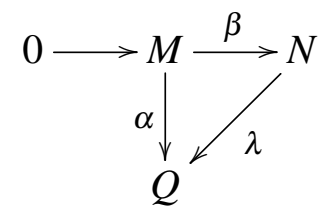

Na língua das categorias, um $R$-modulo $Q$ é injetivo se o functor $\operatorname{Hom}(\cdot, Q)$ é exato.

\subsubsection{Produto tensorial}

Definição 9. Sejam $R$ um anel, $M, N, P R$-módulos. Dizemos que um mapa

$$
\alpha: M \times N \longrightarrow P
$$

é bilinear se é linear em cada variável, isto é, dado $m \in M$ e $n \in N$, os mapas

$$
m^{\prime} \mapsto \alpha\left(m^{\prime}, n\right) \text { e } n^{\prime} \mapsto \alpha\left(m, n^{\prime}\right)
$$

são $R$-lineares. Denotamos o conjunto de todos esses mapas por $\operatorname{Bil}_{R}(M, N ; P)$. É claramente um $R$-módulo, com suma e produto por escalar dada valor a valor. 
Definição 10. Sejam $M, N R$-módulos. Seu Produto tensorial, denotado por $M \otimes_{R} N$ ou simplesmente $M \otimes N$, é construído como o quociente do $R$-módulo livre $R^{\oplus(M \times N)}$ módulo o submódulo gerado pelos seguintes elementos, onde $(m, n)$ represento o elemento $\left.e_{(} m, n\right)$ da base canônica:

$$
\begin{aligned}
\left(m+m^{\prime}, n\right)-(m, n)- & \left(m^{\prime}, n\right) \text { e }\left(m, n+n^{\prime}\right)-(m, n)-\left(m, n^{\prime}\right), \\
& (r m, n)-(m, r n) \\
& (r m, n)-r(m, n) \\
& (m, r n)-r(m, n),
\end{aligned}
$$

para cada $m, m^{\prime} \in M$ e $n, n^{\prime} \in N$ e $r \in R$. A construção de acima da um mapa bilinear canônico

$$
\beta: M \times N \longrightarrow M \otimes_{R} N
$$

Denotaremos por $m \otimes n:=\beta(m, n)$.

Teorema 2 (Propriedade universal do produto tensorial). Sejam $R$ um anel, $M, N R$-módulos. Então $\beta: M \times N \longrightarrow M \otimes_{R} N$ é o exemplo universal de mapa bilinear sobre $M \times N$, isto é, $\beta$ induz um isomorfismo de $R$-módulos,

$$
\theta: \operatorname{Hom}_{R}\left(M \otimes_{R} N, P\right) \stackrel{\sim}{\longrightarrow} \operatorname{Bil}_{R}(M, N ; P) .
$$

Demonstração. Vide [(ALTMAN; KLEIMAN, 2012), Teorema 8.3].

\subsubsection{Anéis e módulos graduados}

Definição 11. Um anel $R$ é $\mathbb{N}$-graduado, se existe uma família de subgrupos $\left\{R_{n}\right\}_{n \in \mathbb{N}}$ de $R$ tal que

(1) $R=\bigoplus_{n \in \mathbb{N}} R_{n}$ (como grupo abeliano), $\mathrm{e}$

(2) $R_{n} \cdot R_{m} \subseteq R_{m+n}$, para todo $n, m \in \mathbb{N}$.

Um elemento não nulo $x \in R_{n}$ é chamado elemento homogêneo de grau $n \mathrm{em} R$.

Observação 4. Todo anel $R$ pode-se considerar $\mathbb{N}$-graduado, com a graduação trivial dada por

$$
R_{n}=\left\{\begin{array}{lll}
R & \text {, se } & n=0 \\
0 & \text {, se } & n \neq 0
\end{array}\right.
$$

Definição 12. Sejam $R$ um anel $\mathbb{N}$-graduado e $M$ um $R$-modulo. Dizemos que $M$ é um $R$-módulo graduado (ou tem uma $R$-graduação) se existe uma família de subgrupos $\left\{M_{n}\right\}_{n \in \mathbb{N}}$ de $M$ tal que

(1) $M=\bigoplus_{n \in \mathbb{N}} M_{n}$ (como grupo abeliano), e 
(2) $R_{n} \cdot M_{m} \subseteq M_{m+n}$, para todo $n, m \in \mathbb{N}$

Se $y \in M-\{0\}$ e $y=y_{n_{1}}+\cdots+y_{n_{k}}$, onde $y_{n_{i}} \in M_{n_{i}}-\{0\}$, os elementos $y_{n_{1}}, \cdots, y_{n_{k}}$ são ditos de componentes homogêneas da $y$. Se $x \in M_{n}-\{0\}$, denotamos deg $x:=n$, o grau do elemento homogêneo $x$.

Se $A$ e $B$ são $R$-módulos graduados, então $A \otimes_{R} B$ é o $R$-módulo graduado tal que $\left(A \otimes_{R} B\right)_{n}:=\bigoplus_{i+j=n} A_{i} \otimes_{R} B_{j}$.

Definição 13 (Homomorfismo de $R$-módulos graduados). Sejam $M$ e $N R$-módulos graduados, e $\varphi: M \longrightarrow N$ um $R$-morfismo é chamado homogêneo de grau $i$, si $\varphi\left(M_{n}\right) \subseteq N_{n+i}$, para cada $i \in \mathbb{N}$.

A partir de agora $R$ sera um anel comutativo (consideraremos a $R$ com a graduação trivial, salvo indicação em contrário) e todos os morfismos de $R$-módulos graduados serão considerados graduados e de grau 0 , salvo indicação em contrário.

Observação 5. Se $M$ é um $R$-módulo graduado, então cada $M_{n}$ é um $R$-módulo $\left(R \cdot M_{n} \subseteq M_{n}\right)$.

Dado um $R$-modulo graduado $M=\bigoplus_{n \in \mathbb{N}} M_{n}$, denotaremos por $M^{*}$ ao $R$-modulo graduado $\bigoplus_{n \in \mathbb{N}} \operatorname{Hom}\left(M_{n}, R\right)$. Se $f: M \longrightarrow N$ é um mapa de $R$-módulos graduados, então $f^{*}: N^{*} \longrightarrow$ $M^{*}$ é o mapa dual definido por $f^{*}\left(n^{*}\right)(m)=n^{*}(f(m))$, onde $n^{*} \in N^{*}$.

Definição 14. Um $R$-álgebra graduada $M=\bigoplus_{n \in \mathbb{N}} M_{n}$ é dita de tipo finito se cada $M_{n}$ é um $R$-modulo finitamente gerado.

Proposição 2. Sejam $M$ e $N R$-módulos graduados os quais são projetivos e de tipo finito. Então

(1) O mapa $\varphi: M \longrightarrow M^{* *}$ definido por $\varphi(x)\left(m^{*}\right)=m^{*}(x)$, onde $x \in M, m^{*} \in M^{*}$ é um isomorfismo, e

(2) o mapa $\vartheta: M^{*} \otimes_{R} N^{*} \longrightarrow\left(M \otimes_{R} N\right)^{*}$ definido por $\vartheta\left(m^{*} \otimes n^{*}\right)(x \otimes y)=m^{*}(x) \otimes n^{*}(y)$, onde $m^{*} \in M^{*}, n^{*} \in N^{*}, x \in A$ e $y \in B$ é um isomorfismo.

Demonstração. Todo $R$-modulo projetivo finitamente gerado é finitamente apresentado, e o resultado segue de uma aplicação direita do Lema 1 (Lema dos cinco).

\section{2 Álgebras e coálgebras graduadas}

Para qualquer par de $R$-módulos graduados $A$ e $B$, seja $T: A \otimes_{R} B \rightarrow B \otimes_{R} A$, o morfismo definido por

$$
T(x \otimes y)=(-1)^{m n} y \otimes x,
$$

onde $x \in A_{m}$, e $y \in B_{n}$. 
Definição 15. Uma $R$-álgebra graduada é um $R$-módulo graduado $A$ junto com um $R$-morfismo chamado unidade $\varepsilon: R \rightarrow A$ e um $R$-morfismo chamado multiplicação $\mu: A \otimes_{R} A \rightarrow A$ tal que as composições

$$
\begin{aligned}
& A \stackrel{\cong}{\longrightarrow} A \otimes_{R} R \stackrel{i d \otimes \varepsilon}{\longrightarrow} A \otimes_{R} A \stackrel{\mu}{\longrightarrow} A \\
& A \stackrel{\cong}{\longrightarrow} A \otimes_{R} R \stackrel{\varepsilon \otimes i d}{\longrightarrow} A \otimes_{R} A \stackrel{\mu}{\longrightarrow} A
\end{aligned}
$$

são as identidades. Uma $R$-álgebra graduada $A$ é associativa se o diagrama

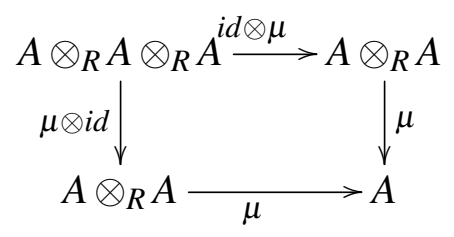

é comutativo. Uma $R$-álgebra graduada é comutativa se o diagrama

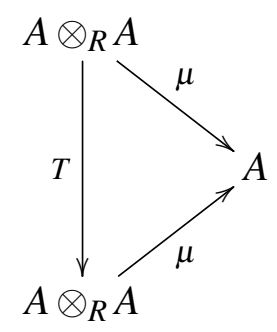

é comutativo.

Definição 16. Uma $R$-coalgebra graduada é um $R$-módulo graduado $A$ junto com a counidade $\eta: A \rightarrow R$ e o comultiplicação $\Delta: A \rightarrow A \otimes_{R} A$ tal que a composição

$$
\begin{aligned}
& A \stackrel{\Delta}{\longrightarrow} A \otimes_{R} A \stackrel{I d \otimes \eta}{\longrightarrow} A \otimes_{R} R \stackrel{\cong}{\longrightarrow} A \\
& A \stackrel{\Delta}{\longrightarrow} A \otimes_{R} A \stackrel{\eta \otimes I d}{\longrightarrow} R \otimes_{R} A \stackrel{\cong}{\longrightarrow} A
\end{aligned}
$$

são as identidades. Uma $R$-coalgebra graduada $A$ é associativa, se o diagrama

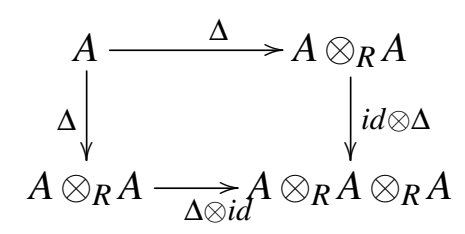

é comutativo. Uma $R$-coalgebra graduada $A$ é comutativa se o diagrama

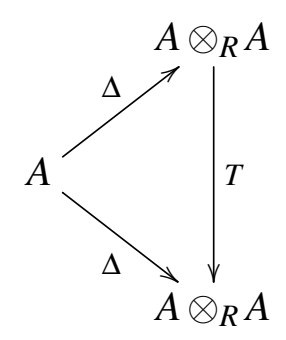

é comutativo. 
Exemplo 3. O anel $R$ tem uma estrutura natural de $R$-álgebra graduada ( $R$ com graduação trivial) com a unidade $\varepsilon$ dada pela identidade $\varepsilon=i d: R \rightarrow R$ e a multiplicação $\mu$ dado pelo isomorfismo $R \otimes_{R} R \stackrel{\cong}{\longrightarrow} R$. Além disso, o anel $R$ tem também uma estrutura de $R$-coalgebra graduada ( $R$ com graduação trivial) com a counidade $\eta$ dada pela identidade $\eta=i d: R \rightarrow R$ e o coproduto $\Delta$ dado pelo isomorfismo $R \stackrel{\cong}{\longrightarrow} \otimes_{R} R$. Como a multiplicação em $R$ é associativa, então $R$ é um álgebra e uma coalgebra graduada associativa. É claro que se $R$ é comutativo, essas estruturas também são comutativas onde $T: R \otimes_{R} R \longrightarrow R \otimes_{R} R$ bem dado por $a \otimes b \mapsto b \otimes a$.

Teorema 3. Se $A$ é $R$-álgebra graduada com multiplicação $\mu: A \otimes_{R} A \longrightarrow A$, e o $R$-modulo $A$ é projetivo e de tipo finito, então $A^{*}$ é uma $R$-coalgebra com comultiplicação $\Delta:=\mu^{*}: A^{*} \longrightarrow$ $A^{*} \otimes_{R} A^{*}, \mathrm{e}$

(1) $A$ é associativo, se e somente se $A^{*}$ é associativo,

(2) $A$ é aumentado, se e somente se $A^{*}$ é aumentado,

(3) A é comutativo, se e somente se $A^{*}$ é comutativo.

Demonstração. Segue imediato da definição e a Proposição 2.

Teorema 4. Se $A$ é $R$-coálgebra graduada com comultiplicação $\Delta: A \longrightarrow A \otimes_{R} A$, e o $R$-modulo $A$ é projetivo e de tipo finito, então $A^{*}$ é uma $R$-álgebra com multiplicação $\mu:=\Delta^{*}: A^{*} \otimes_{R} A^{*} \longrightarrow$ $A^{*}, \mathrm{e}$

(1) $A$ é associativo, se e somente se $A^{*}$ é associativo,

(2) A é aumentado, se e somente se $A^{*}$ é aumentado,

(3) A é comutativo, se e somente se $A^{*}$ é comutativo.

Demonstração. Segue imediato da definição e a Proposição 2.

Observação 6. Se $A$ é projetivo e de tipo finito então $A^{*}$ é projetivo e de tipo finito. A reciproca não sempre é verdadeira

Definição 17. Se $\left(A, \mu_{A}, \varepsilon_{A},\right)$ e $\left(B, \mu_{B}, \varepsilon_{B}\right)$ um par de $R$-álgebras graduadas, então $A \otimes_{R} B$ é a $R$-álgebra graduada com multiplicação $\mu_{A \otimes_{R} B}:\left(A \otimes_{R} B\right) \otimes_{R}\left(A \otimes_{R} B\right) \longrightarrow A \otimes_{R} B$ tal que o diagrama

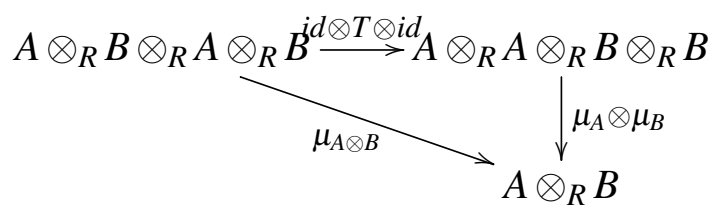


é comutativo, e com unidade $\varepsilon_{A \otimes_{R} B}: R \longrightarrow A \otimes_{R} B$ tal que o diagrama

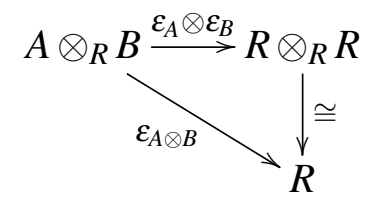

é comutativo.

Se $\left(A, \Delta_{A}, \eta_{A}\right)$ e $\left(B, \Delta_{B}, \eta_{B}\right)$ um par de $R$-coálgebras graduadas, então $A \otimes_{R} B$ é a $R$-coálgebra graduada com coproduto $\Delta_{A \otimes_{R} B}: A \otimes_{R} B \longrightarrow\left(A \otimes_{R} B\right) \otimes_{R}\left(A \otimes_{R} B\right)$ tal que o diagrama

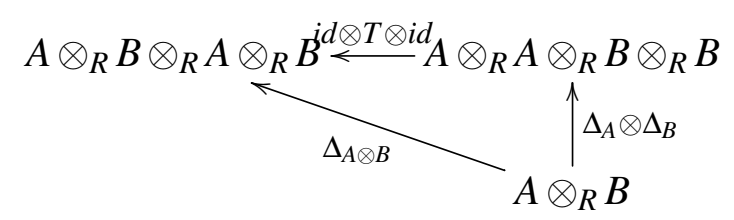

é comutativo, e com counidade $\eta_{A \otimes_{R} B}: R \longrightarrow A \otimes_{R} B$ tal que o diagrama

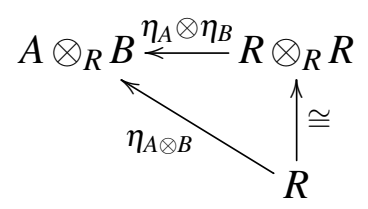

é comutativo.

Definição 18. (1) Seja $\left(A, \mu_{A}, \varepsilon_{A}\right),\left(B, \mu_{B}, \varepsilon_{B}\right)$ duas $R$-álgebras graduadas. Dizemos que um $R$-morfismo $f: A \longrightarrow B$ é um morfismo de álgebras se os seguintes diagramas são comutativos
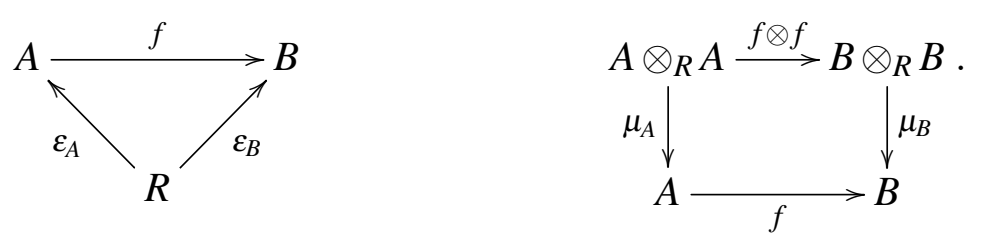

(2) Seja $\left(C, \Delta_{c}, \eta_{C}\right),\left(D, \Delta_{D}, \eta_{D}\right)$ duas $R$-coalgebras graduadas. Dizemos que um $R$-morfismo $g: C \longrightarrow D$ é um morfismo de coalgebras se os seguintes diagramas são comutativos
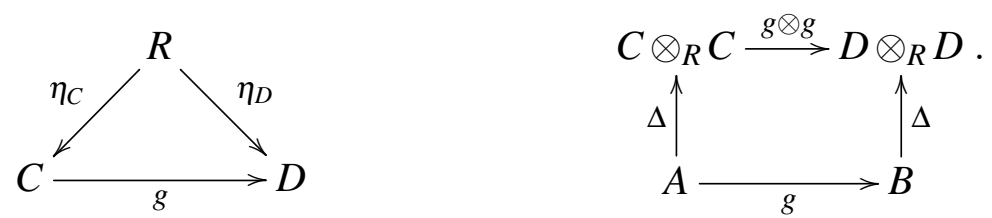

Proposição 3. Seja $A$ um $R$-módulo graduado que tem uma estrutura de $R$-álgebra $(A, \mu, \varepsilon)$ e uma estrutura de $R$-coálgebra $(A, \Delta, \eta)$. As seguintes afirmações são equivalentes:

(i) Os mapas $\mu$ e $\varepsilon$ são morfismos de $R$-coálgebras.

(ii) Os mapas $\Delta$ e $\eta$ são morfismo de $R$-álgebras. 
Demonstração. Segue imediato dos seguintes diagramas
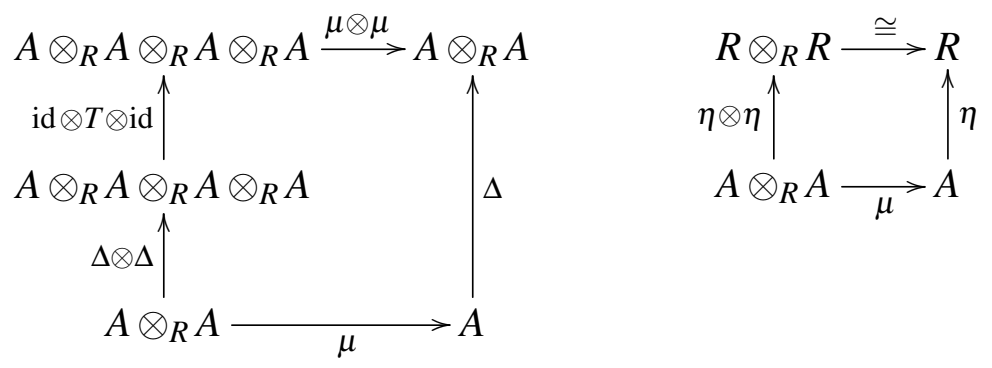

$\mathrm{e}$
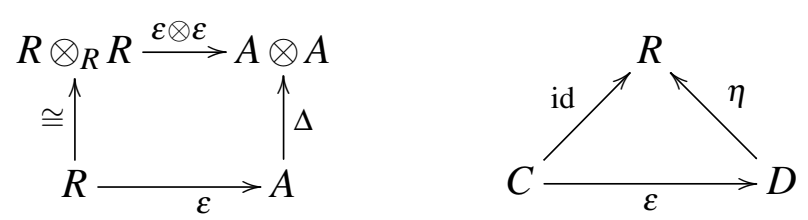

\subsection{1 Álgebra de Hopf}

Definição 19. Um $R$-módulo graduado $A$, junto com uma unidade $\varepsilon$, uma counidade $\eta$, um produto $\mu$, e um coproduto $\Delta$, é uma Álgebra de Hopf se,

(i) $(A, \mu, \varepsilon)$ é uma $R$-álgebra associativa,

(ii) $(A, \Delta, \eta)$ é uma $R$-coálgebra associativa,

(iii) $\mu$ e $\varepsilon$ são morfismos de $R$-coálgebras,

(iv) $\Delta$ e $\eta$ são morfismos de $R$-álgebras.

Observação 7. Pela proposição anterior, os itens (iii) e (iv) são equivalentes.

Pela definição temos que o diagrama

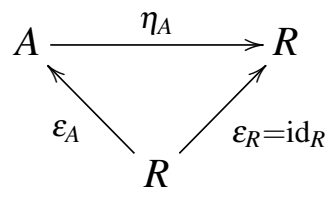

é comutativo, pois $\eta_{A}$ é um morfismo de $R$-álgebras, então $A$ pode ser considerado como a suma direita

$$
A=\operatorname{Im}(\varepsilon) \bigoplus \operatorname{ker}(\eta)
$$

e os $R$-módulos $\operatorname{ker}(\eta)$ e coker $(\varepsilon)$ são isomorfos e será denotado por $\bar{A}$. Assim

$$
0 \longrightarrow \bar{A} \longrightarrow A \stackrel{\eta}{\longrightarrow} R \longrightarrow 0 \quad \text { e } \quad 0 \longrightarrow R \stackrel{\varepsilon}{\longrightarrow} A \longrightarrow \bar{A} \longrightarrow 0
$$


são sequências curtas exatas. Além disso o triangulo superior direito do diagrama

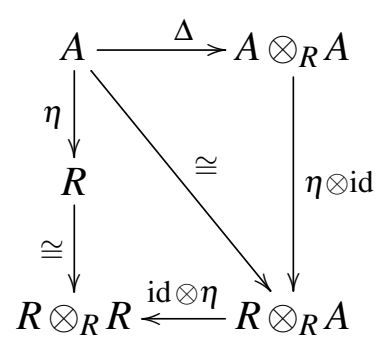

é comutativo desde que $\eta: A \longrightarrow R$ é a counidade da $R$-coalgebra. A parte inferior do diagrama é comutativa, pois $\eta: A \longrightarrow R$ é um morfismo de $R$-álgebras e $\eta \otimes \eta=(\eta \otimes i d) \circ(i d \otimes \eta)$.

Definição 20. Uma álgebra de Hopf $A$ é conexa se uma das seguintes condições equivalentes é satisfeita:

(1) $\varepsilon: R \stackrel{\cong}{\longrightarrow} A_{0}$

(2) $\eta: A_{0} \stackrel{\cong}{\longrightarrow} R$.

Portanto, se $A$ é uma álgebra de Hopf conexa, $\bar{A}=\bigoplus_{n>0} A_{n}$. Vemos que para qualquer álgebra de Hopf $A$, temos $A=R \bigoplus \bar{A}$ como um $R$-modulo,

$$
A \otimes_{R} A=R \otimes_{R} R+R \otimes_{R} \bar{A}+\bar{A} \otimes_{R} R+\bar{A} \otimes_{R} \bar{A}
$$

e para cada $x \in A$, temos

$$
\Delta(x)=x \otimes 1+1 \otimes x+\sum x_{i}^{\prime} \otimes x_{i}^{\prime \prime}
$$

$\operatorname{com} x_{i}^{\prime}, x_{i}^{\prime \prime} \in \bar{A}$. Se $A$ é conexa, então $0<\operatorname{deg} x_{i}^{\prime}<\operatorname{deg} x$.

Observação 8. Se $R$ é um corpo e $A$ uma $R$-álgebra de tipo finito então como $A=R \bigoplus \bar{A}$ temos que $A^{*}=R \oplus \bar{A}^{*}$ e assim temos $\overline{A^{*}}=\bar{A}^{*}$.

Definição 21. Se $A$ é uma álgebra de Hopf definimos $Q(A)$ como o conucleo do mapa induzido pelo produto $\mu: \bar{A} \otimes_{R} \bar{A} \longrightarrow \bar{A}$, isto é $Q(A)=\bar{A} / \bar{A}^{2}$. Os elementos de $Q(A)$ são ditos de elementos indecomponíveis de $A$. E assim, temos a seguinte sequência exata

$$
\bar{A} \otimes_{R} \bar{A} \stackrel{\mu}{\longrightarrow} \bar{A} \longrightarrow Q(A) \longrightarrow 0
$$

Definição 22. Se $A$ é uma álgebra de Hopf definimos $P(A)$ como o núcleo do mapa

$$
\begin{aligned}
\delta: \bar{A} & \rightarrow \bar{A} \otimes_{R} \bar{A} \\
x & \mapsto \sum x_{i}^{\prime} \otimes x_{i}^{\prime \prime},
\end{aligned}
$$

onde $\sum x_{i}^{\prime} \otimes x_{i}^{\prime \prime}$ é como em (1.5). Os elementos da $P(A)$ são ditos de elementos primitivos de $A$. E assim, temos a seguinte sequência exata

$$
0 \longrightarrow P(A) \longrightarrow \bar{A} \stackrel{\delta}{\longrightarrow} \bar{A} \otimes_{R} \bar{A}
$$


Observação 9. Se $x \in \bar{A}$, que por (1.5) temos $\Delta(x)=x \otimes 1+1 \otimes x+\delta(x)$. E portanto, $x \in \bar{A}$ é primitivo se e somente se $\Delta(x)=1 \otimes x+x \otimes 1$.

Proposição 4. Seja $K$ um corpo e $A$ uma álgebra de Hopf sobre $K$ a qual é um $K$-modulo de tipo finito, então

(1) $P\left(A^{*}\right)=Q(A)^{*}$

(2) $Q\left(A^{*}\right)=P(A)^{*}$

Demonstração. Temos a seguintes sequências exatas

$$
\bar{A} \otimes_{R} \bar{A} \stackrel{\mu}{\longrightarrow} \bar{A} \longrightarrow Q(A) \longrightarrow 0
$$

e

$$
0 \longrightarrow P(A) \longrightarrow \bar{A} \stackrel{\delta}{\longrightarrow} \bar{A} \otimes_{R} \bar{A}
$$

Dualizando e pela Proposição 2

$$
0 \longrightarrow Q(A)^{*} \longrightarrow \bar{A}^{*} \stackrel{\mu^{*}}{\longrightarrow} \bar{A}^{*} \otimes_{R} \bar{A}^{*}
$$

e

$$
\bar{A}^{*} \otimes_{R} \bar{A}^{*} \stackrel{\delta^{*}}{\longrightarrow} \bar{A}^{*} \longrightarrow P(A)^{*} \longrightarrow 0
$$

Por tanto, da Observação 8 temos

$$
P\left(A^{*}\right)=Q(A)^{*}
$$

e

$$
Q\left(A^{*}\right)=P(A)^{*}
$$

Proposição 5. Seja $K$ um corpo, $f: A \longrightarrow B$ um mapa de álgebras de Hopf conexas sobre $K, P(f): P(A) \longrightarrow P(B)$, e $Q(f): Q(A) \longrightarrow Q(B)$ são os mapas induzidos pela $f$. Isto é, os seguintes diagramas

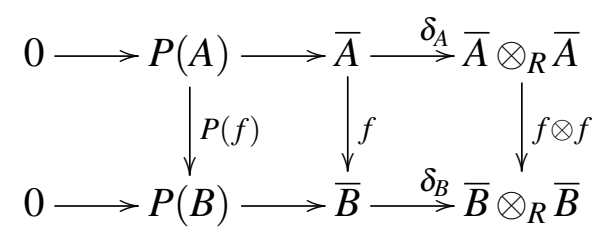

e

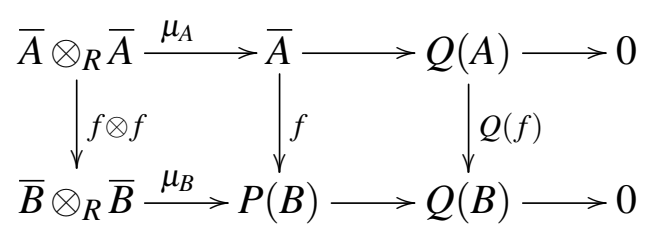

são comutativos. Então 
(1) $f$ é um monomorfismo se e somente se $P(f)$ é um monomorfismo,e

(2) $f$ é um epimorfismo se e somente se $Q(A)$ é um epimorfismo.

Demonstração. Vide [(MILNOR; MOORE, 1959), Proposição 3.8].

\section{3 Álgebras de Hopf sobre corpos}

Agora consideramos a $R$ como um corpo, o qual denotaremos por $K$.

Definição 23. Se $L$ é um espaço vetorial graduado sobre $K$, denotamos por $T(L)=\bigoplus_{n>0} T^{n}(L)$, onde $T^{n}(L)=L \otimes_{K} L \otimes_{K} \cdots \otimes_{K} L$ (n-vezes), a álgebra associativa livre, ou álgebra tensor, gerada pela $L$, onde a multiplicação é dada pela concatenação de elementos e a unidade pela inclusão. Denotamos também por $\Lambda(L)$ a álgebra quociente da $T(L)$ pelo ideal gerado pelos elementos da forma $x \otimes y-(-1)^{m n} y \otimes x$, onde $x \in L_{m}$ e $y \in L_{n}$. A álgebra $\Lambda(L)$ a qual é dita de álgebra associativa comutativa livre gerada pela $L$. Se $L$ é tal que $L_{n}=0$ para $n$ par, denotamos por $E(L)$ a álgebra quociente da $T(L)$ pelo ideal gerado pelos elementos da forma $x^{2}$, onde $x \in L$. A álgebra $E(L)$ é dita de álgebra de Grassmann ou álgebra exterior gerada pela $L$.

Observação 10. Se $B$ é qualquer álgebra associativa e $L$ como antes, e $f: L \longrightarrow B$ um mapa de espaços vetoriais graduados, então existe um único mapa $\widetilde{f}: T(L) \longrightarrow B$ tais que $\widetilde{f} \circ i=f$ onde $i$ é a inclusão de $L$ em $T(L)$. Além disso, se $B$ é comutativa o mapa $\widetilde{f}$ se fatora de maneira única pela $\Lambda(L)$. Assim tanto o functor $T($.) e o functor $\Lambda($.) são functores adjuntos a esquerda do functor esquecedor, e por tanto eles preservam coprodutos (objetos de categorias)

Se $L=L^{\prime} \oplus L^{\prime \prime}$, então $T(L)$ é o produto livre de $T\left(L^{\prime}\right)$ e $T\left(L^{\prime \prime}\right)$ (coproduto na categoria das álgebras associativas), e $\Lambda(L)=\Lambda\left(L^{\prime}\right) \otimes \Lambda\left(L^{\prime \prime}\right)$ (coproduto na categoria das álgebras comutativas associativas). Mais ainda, se $L$ é um limite direito de espaços vetoriais sobre $K$, então $T(L)$ é o limite direito das correspondentes álgebras associativas livres, e $\Lambda(L)$ é o limite direito das álgebras comutativas associativas livres.

Proposição 6. Se $B$ é uma álgebra de Hopf conexa sobre um corpo $K$ de característica zero, então o mapa natural verifica o seguinte:

(1) $P(B) \longrightarrow Q(B)$ é monomorfismo se e somente se o produto em $B$ é comutativo e associativo, e

(2) $P(B) \longrightarrow Q(B)$ é sobrejetivo se e somente se o coproduto da $B$ é comutativo e associativo. 
Proposição 7. Se $B$ é uma álgebra de Hopf conexa com produto associativo e seja $L=Q(B)$, existe um mapa $\bar{\Delta}: T(L) \longrightarrow T(L) \otimes_{K} T(L)$ com o qual $T(L)$ torna-se uma álgebra de Hopf, e um mapa $f: T(L) \longrightarrow B$ de álgebras de Hopf tais que

(1) $f$ é um sobrejetor e

(2) $Q(f): Q(T(L)) \longrightarrow Q(B)$ é um isomorfismo.

Demonstração. Vide [(MILNOR; MOORE, 1959), Proposição 4.4]

Proposição 8. Se $B$ é uma álgebra de Hopf com produto comutativo e associativo e $L=Q(B)$, existe um mapa $\bar{\Delta}: \Lambda(L) \longrightarrow \Lambda(L) \otimes_{K} \Lambda(L)$ com o qual $\Lambda(L)$ torna-se uma álgebra de Hopf, e um mapa $g: \Lambda(L) \longrightarrow \Lambda(L) \otimes_{K} \Lambda(L)$ de álgebras de Hopf tais que

(1) g e um epimorfismo, e

(2) $Q(g): Q(\Lambda(L) \longrightarrow Q(B)$ é um isomorfismo.

Demonstração. Vide [(MILNOR; MOORE, 1959), Proposição 4.5]

Teorema 5 (Hopf-Leray). Se $B$ é uma álgebra de Hopf conexa com produto comutativo e associativo sobre um corpo $K$ de característica zero, e $L=Q(B)$, então $B$ é isomorfo com $\Lambda(L)$ como álgebra.

Demonstração. Vide [(MILNOR; MOORE, 1959), Teorema 4.6]

Teorema 6 (Samelson-Leray). Seja $B$ uma álgebra de Hopf conexa sobre o corpo $K$, com produto comutativo associativo e coproduto associativo, e suponhamos que $B$ é gerada como álgebra por um conjunto de elementos $\left\{x_{i}\right\}$ tais que $x_{i}^{2}=0$ e o grau de $x_{i}$ é impar. Seja $L=P(B)$, e definimos um coproduto em $E(L)$, o álgebra exterior gerada pela $L$, definindo $\Delta(x)=x \otimes 1+1 \otimes x$ para $x \in L$. Então $B$ é isomorfa com $E(L)$ como uma álgebra de Hopf. Em particular $Q(B) \longrightarrow$ $Q(E(L))=L$ é um isomorfismo.

Demonstração. Vide [(MILNOR; MOORE, 1959), Teorema 4.10]

\section{4 Álgebras de Lie e álgebras de Hopf}

Nesta secção todas as álgebras serão definidas sobre um corpo $K$, e devemos assumir que tem produto associativo com unidade. Mais ainda, por uma álgebra de Hopf entenderemos por uma álgebra de Hopf associativa, em outras palavras temos produto e coproduto associativo. Finalmente um espaço vetorial significara um espaço vetorial sobre $K$ graduado.

Definição 24. Se $A$ é uma álgebra, definimos $[\cdot, \cdot]: A \otimes_{K} A \longrightarrow A$ por $[x, y]=x y-(-1)^{n m} y x$, onde $x \in A_{n}$ e $y \in A_{m}$. 
Uma álgebra de Lie is um espaço vetorial $L$ munido de um mapa de espaços vetoriais $[\cdot, \cdot]: L \otimes_{K} L \longrightarrow L$ tais que para alguma álgebra $A$ existe um monomorfismo de espaços vetoriais $f: L \longmapsto A$ tais que $f([x, y])=[f(x), f(y)]$ para $x, y \in L$.

Observação 11. Pela definição anterior temos que toda álgebra associativa é uma álgebra de Lie com $[x, y]=x y-(-1)^{n m} y x$, onde $x \in A_{n}$ e $y \in A_{m}$ e $f=i d_{A}$.

Lema 2. Se $L$ é uma álgebra Lie, $x \in L_{n}, y \in L_{m}, z \in L_{r}$, então

(1) $[x, y]=(-1)^{n m+1}[y, x]$,

(2) $(-1)^{n r}[x,[y, z]]+(-1)^{m n}[y,[z, x]]+(-1)^{r n}[z,[x, y]]=0$,

(3) $[x,[x, x]]=0, \mathrm{e}$

(4) $[x, x]=0$ se $n$ é par ou $\operatorname{char}(K)=2$.

Demonstração. Imediato pela definição.

Proposição 9. Se $L$ é uma álgebra de Lie, existe uma álgebra $U(L)$ e um monomorfismo $i: L \longrightarrow U(L)$ de álgebras de Lie tais que se $A$ é uma álgebra, e $f: L \longrightarrow A$ é um mapa de álgebras de Lie, existe um único mapa de álgebras $\bar{f}: U(L) \longrightarrow A$ tais que $\bar{f} \circ i=f$. Se $V(L)$ e $j: L \longrightarrow V(L)$ são quaisquer outras álgebra e monomorfismo de álgebra de Lie, então $\bar{j}: U(L) \longrightarrow V(L)$ é um isomorfismo

Definição 25. Se $L$ é uma álgebra de Lie, e i : $L \longrightarrow U(L)$ é um monomorfismo satisfazendo as condições anteriores, $U(L)$ é dito de álgebra universal de $L$.

Proposição 10. Se $A$ é uma álgebra de Hopf, então $P(A)$ é uma subálgebra de $A$.

Demonstração. Sejam $x \in P(A)_{n}, y \in P(A)_{m}$, então

$$
\begin{gathered}
\Delta(x)=x \otimes 1+1 \otimes x, \Delta(y)=y \otimes 1+1 \otimes y, \\
\Delta(x y)=\Delta(x) \Delta(y)=x y \otimes 1+1 \otimes x y+x \otimes y+(-1)^{n m} y \otimes x, \\
\Delta(y x)=\Delta(y) \Delta(x)=y x \otimes 1+1 \otimes y x+y \otimes x+(-1)^{n m} x \otimes y, \\
\Delta([x, y])=[x, y] \otimes 1+1 \otimes[x, y],
\end{gathered}
$$

então $[x, y] \in P(A)_{n+m}$, e portanto $P(A)$ é uma subálgebra de Lie de $A$.

Proposição 11. Se $L$ é uma álgebra de Lie, existe um único mapa $\bar{\Delta}: U(L) \longrightarrow U(L) \otimes_{K} U(L)$ tais que $U(L)$ é uma álgebra de Hopf, e $L \subset P(U(L))$. 
Definição 26. Uma álgebra de Lie $L$ é comutativa se $[x, y]=0$ para $x, y \in L$. Se $L^{\prime}$ e $L^{\prime \prime}$ são álgebras de Lie, então $L^{\prime} \bigoplus L^{\prime \prime}$ é a álgebra de Lie munido do mapa $\left[\left(x^{\prime}, x^{\prime \prime}\right),\left(y^{\prime}, y^{\prime \prime}\right)\right]:=\left(\left[x^{\prime}, y^{\prime}\right],\left[x^{\prime \prime}, y^{\prime \prime}\right]\right)$ para $x^{\prime}, y^{\prime} \in L^{\prime}, x^{\prime \prime}, y^{\prime \prime} \in L^{\prime \prime}$.

Observação 12. (1) Se $L$ é uma álgebra de Lie comutativa, então o ideal gerado pelos elementos da forma $x y-(-1)^{n m} y x-[x, y] \operatorname{com} x \in L_{n}, y \in L_{m}$ em $T(L)$, é o mesmo que o ideal gerado por $x y-(-1)^{n m} y x \operatorname{com} x \in L_{n}, y \in L_{m}$ e assim temos que $U(L)=\Lambda(L)$.

(2) Se $L$ e $M$ são álgebras de Lie, então $U(L \bigoplus M)=U(L) \otimes U(M)$, pois o functor $U($.) é um functor adjunto a esquerda do functor esquecedor.

Teorema 7. Seja $A$ um álgebra de Hopf conexa com coproduto comutativo sobre um corpo $K$ de característica zero. Então o mapa $U(P(A)) \longrightarrow A$,induzido pelo mapa natural $P(A) \longrightarrow A$, é um isomorfismo de álgebras de Hopf.

Demonstração. Vide [(MILNOR; MOORE, 1959), Teorema 5.22]

O teorema anterior nos diz quando uma álgebra de Hopf sobre um corpo de característica zero é gerada (como álgebra) pelos elementos primitivos.

Definição 27. Se $B$ é um espaço vetorial, denotamos por $L(B)$ a álgebra de Lie em $T(B)$ gerado pelo espaço vetorial $B$.

Proposição 12. Se $B$ é um espaço vetorial sobre $K$, então $U(L(B))=T(B)$.

Demonstração. Vide [(MILNOR; MOORE, 1959), Proposição 5.16]

Definição 28. Se $L$ é uma álgebra de Lie e $U(L)$ sua álgebra universal, definimos uma filtração sobre $U(L)$ definindo

$$
F_{0} U(L)=K, \quad F_{1} U(L)=K+L, \quad \text { e } \quad F_{n} U(L)=\left(F_{1} U(L)\right)^{n}
$$

Seja $E^{0}=\sum_{r, s} E_{r, s}^{0} U(L)$ onde $E_{r, s}^{0}=\left(F_{r} U(L) / F_{r-1} U(L)\right)_{r+s}$, e $\quad E_{r}^{0} U(L)=\sum_{s} E_{r, s}^{0} U(L)$.

Teorema 8 (Poincaré-Birkhoff-Witt). Se $K$ é um corpo de característica zero, $L$ uma álgebra de Lie sobre $K$, entao o morfismo natural

$$
E^{0}(\Lambda(L)) \longrightarrow E^{0}(U(L))
$$

é um isomorfismo de álgebras de Hopf bi-graduadas.

Demonstração. Vide [(MILNOR; MOORE, 1965), Teorema 5.15]

Corolário 1. Se $L$ é uma álgebra de Lie sobre $K$. Então existe um (não natural) isomorfismo de $K$-módulos entre $\Lambda(L)$ e $U(L)$. 
Definição 29. Uma $K$-álgebra graduada, o produto é dito de estritamente comutativa se é comutativo e se $x^{2}=0$ para todo $x$ homogêneo em $A$ de grau impar.

Teorema 9 (Samelson-Leray). Seja $A$ uma álgebra de Hopf conexa sobre um corpo $K$, com produto estritamente comutativo e $Q(A)_{n}=0$ para $n$ par. Então, o morfismo natural $E(P(A)) \longrightarrow$ $A$, induzido pelo mapa natural $P(A) \longrightarrow A$, é um isomorfismo de álgebras de Hopf.

Demonstração. Vide [(MILNOR; MOORE, 1965), Teorema 7.20] 
CAPÍTULO

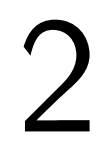

\section{COHOMOLOGIA DE GRUPOS E O ISOMORFISMO DE VAN EST}

Neste capitulo daremos as definições das teoria de homologia e cohomologia que usaremos mas adiante.

\subsection{Homologia e Cohomologia de um complexo}

Definição 30. Seja $R$ um anel. Um sequência de $R$-homomorfismos

$$
\ldots \longrightarrow A_{n+1} \stackrel{d_{n+1}}{\longrightarrow} A_{n} \stackrel{d_{n}}{\longrightarrow} A_{n-1} \longrightarrow \ldots \longrightarrow A_{2} \stackrel{d_{2}}{\longrightarrow} A_{1} \stackrel{d_{1}}{\longrightarrow} A_{0} \longrightarrow 0
$$

é dita de complexo de cadeias se $d_{n} \circ d_{n+1}=0$, isto é $\operatorname{Im}\left(d_{n+1}\right) \subset \operatorname{ker}\left(d_{n}\right)$, para todo $n \geq 0$. Denotamos este complexo por $\left(A_{\bullet}, d_{\bullet}\right)$ ou simplesmente por $A_{\bullet}$, os $R$-homomorfismos $d_{n}$ são ditos de diferencial ou operador bordo de $A$.. Dualmente, uma sequência de $R$-homomorfismos

$$
0 \longrightarrow A^{0} \stackrel{d^{0}}{\longrightarrow} A^{1} \stackrel{d^{1}}{\longrightarrow} \ldots \longrightarrow A^{n} \stackrel{d^{n}}{\longrightarrow} A^{n+1} \stackrel{d^{n+1}}{\longrightarrow} A^{n+2} \longrightarrow \ldots
$$

é dita de complexo de cocadeias se $d^{n+1} \circ d^{n}=0$, isto é $\operatorname{Im}\left(d^{n}\right) \subset \operatorname{ker}\left(d^{n+1}\right)$, para todo $n \geq 0$. Denotamos este complexo por $\left(A^{\bullet}, d^{\bullet}\right)$ ou simplesmente por $A^{\bullet}$, os $R$-homomorfismos $d^{n}$ são ditos de diferencial ou operador cobordo de $A^{\bullet}$.

Definição 31. Sejam $A_{\bullet}^{1}$ e $A_{\bullet}^{2}$ dois complexos de cadeias. Uma família de $R$-homomorfismos $\left\{f_{n}: A_{n}^{1} \longrightarrow A_{n}^{2}\right\}_{n \in \mathbb{N}}$ é dita de morfismo entre complexos de cadeias se $f_{n} \circ d_{n+1}^{1}=d_{n+1}^{2} \circ f_{n+1}$, para todo $n \geq 0$. Denotamos este morfismo por $f_{\bullet}$. Dualmente, sejam $B_{1}^{\bullet}$ e $B_{2}^{\bullet}$ dois complexos de cocadeias. Uma família de $R$-homomorfismos $\left\{g^{n}: B_{1}^{n} \longrightarrow B_{2}^{n}\right\}_{n \in \mathbb{N}}$ é dita de morfismo entre complexos de cocadeias se $g^{n+1} \circ d_{1}^{n}=d_{2}^{n} \circ g^{n}$, para todo $n \geq 0$. Denotamos este morfismo por $g^{\bullet}$.

Definição 32 (Homologia de um complexo). Seja $A \bullet$ um complexo de cadeias , denotamos por 
$C_{n}\left(A_{\bullet}\right):=A_{n}$, cujos elementos são chamados $n$-cadeias,

$Z_{n}\left(A_{\bullet}\right):=\operatorname{ker}\left(d_{n}\right)$, cujos elementos são chamados $n$-ciclos,

$B_{n}\left(A_{\bullet}\right):=\operatorname{Im}\left(d_{n+1}\right)$, cujos elementos são chamados $n$-bordos ou $n$-ciclos triviais,

$H_{n}\left(A_{\bullet}\right):=Z_{n}\left(A_{\bullet}\right) / B_{n}\left(A_{\bullet}\right)$

O grupo abeliano $H_{n}\left(A_{\bullet}\right)$ é dito de $n$-ésimo grupo de homologia do complexo $A_{\bullet}$.

De maneira dual podemos definir os grupos de cohomologia de um complexo de cocadeias, como segue.

Definição 33 (Cohomologia de um complexo). Seja $B^{\bullet}$ um complexo de cocadeias, denotamos por

$C^{n}\left(B^{\bullet}\right)=B^{n}$, cujos elementos são chamados $n$-cocadeias,

$Z^{n}\left(B^{\bullet}\right)=\operatorname{ker}\left(d^{n}\right)$, cujos elementos são chamados $n$-cociclos,

$B^{n}\left(B^{\bullet}\right)=\operatorname{Im}\left(d^{n-1}\right)$, cujos elementos são chamados $n$-cobordos ou $n$-cociclos triviais,

$H^{n}\left(B^{\bullet}\right)=Z^{n}\left(B^{\bullet}\right) / B^{n}\left(B^{\bullet}\right)$

O grupo abeliano $H^{n}\left(B^{\bullet}\right)$ é dito de $n$-ésimo grupo de cohomologia do complexo $B^{\bullet}$.

Observação 13. Todo morfismo entre complexos de cadeias $f_{\bullet}=\left\{f_{n}\right\}: A_{\bullet}^{1} \longrightarrow A_{\bullet}^{2}$ define naturalmente uma sequencia de morfismos entre os grupos de homologia, a dizer

$$
\begin{aligned}
\left(f_{n}\right)_{*}: H_{n}\left(A_{\bullet}^{1}\right) & \rightarrow H_{n}\left(A_{\bullet}^{2}\right) \\
{[x] } & \mapsto\left[f_{n}(x)\right] .
\end{aligned}
$$

Dualmente, todo morfismo entre complexos de cocadeias $g^{\bullet}=\left\{g^{n}\right\}: B_{1}^{\bullet} \longrightarrow B_{2}^{\bullet}$ define naturalmente uma sequencia de morfismos entre os grupos de cohomologia, a dizer

$$
\begin{aligned}
\left(g^{n}\right)^{*}: H^{n}\left(B_{1}^{\bullet}\right) & \rightarrow H^{n}\left(B_{2}^{\bullet}\right) \\
{[x] } & \mapsto\left[g^{n}(x)\right] .
\end{aligned}
$$

Definição 34. Sejam $A_{\bullet}^{1}$ e $A_{\bullet}^{2}$ e dois morfismo de complexos $f_{\bullet}, h_{\bullet}$ de $A_{\bullet}^{1}$ em $A_{\bullet}^{2}$, os morfismos são chamados homotópicos se existe uma homotopia entre $f_{\bullet}$ e $h_{\bullet}$, isto é uma sequencia de $R$-homomorfismos $s_{n}: A_{n}^{1} \longrightarrow A_{n+1}^{2}$, para cada $n \geq 1$, tais que

(1) $f_{0}-h_{0}=d_{1}^{2} \circ s_{0}$

(2) $f_{n}-h_{n}=s_{n-1} \circ d_{n}^{1}+d_{n+1}^{2} \circ s_{n}$ para todo $n \geq 1$. 
Seja $C_{\bullet}$ um complexo, uma homotopia entre os morfismos de complexos $I d_{C_{\bullet}}$ e $0_{C_{\bullet}}$ é chamada contratante.

Dizemos que dois complexos $A_{\bullet}^{1}$ e $A_{\bullet}^{2}$ são homotopicamente equivalentes se existem dois morfismos $f_{\bullet}: A_{\bullet}^{1} \longrightarrow A_{\bullet}^{2}$ e $g_{\bullet}: A_{\bullet}^{2} \longrightarrow A_{\bullet}^{1}$ tais que os morfismos $f_{\bullet} \circ g_{\bullet}$ e $g_{\bullet} \circ f_{\bullet}$ são homotópicos a $i d_{A_{\bullet}^{2}}$ e $i d_{A_{\bullet}^{1}}$ respectivamente. Dualmente, podemos dar as definições anteriores para o caso de complexos de cocadeias.

Observação 14. É imediato mostrar que dois morfismos de complexos $f_{\bullet}$ e $h \bullet$ que são homotópicos, definem o mesmo morfismo $\left(f_{n}\right)_{*}$ e $\left(g_{n}\right)_{*}$, isto é $\left(f_{n}\right)_{*}=\left(g_{n}\right)_{*}$.

Definição 35. Uma resolução projetiva de um $R$-modulo $M$ é um complexo de $R$-módulos

$$
P_{\bullet}: \ldots \longrightarrow P_{2} \stackrel{d_{2}}{\longrightarrow} P_{1} \stackrel{d_{1}}{\longrightarrow} P_{0} \longrightarrow 0
$$

onde cada $P_{i}$ é um $R$-modulo projetivo e existe um $R$-homomorfismo $\sigma$ tais que o complexo aumentado

$$
P_{\bullet} \rightarrow M: \ldots \longrightarrow P_{2} \stackrel{d_{2}}{\longrightarrow} P_{1} \stackrel{d_{1}}{\longrightarrow} P_{0} \stackrel{\sigma}{\longrightarrow} M \longrightarrow 0,
$$

é exato, isto é, é uma sequência exata.

Exemplo 4. Sejam $\mathfrak{g}$ uma álgebra de Lie sobre $K$ e $V_{p}(\mathfrak{g}):=U(\mathfrak{g}) \otimes_{K} \Lambda^{p} \mathfrak{g}$, e como $\Lambda^{p} \mathfrak{g}$ é um $K$-modulo livre, então $V_{p}(\mathfrak{g})$ é um $U(\mathfrak{g})$-modulo livre. Por convenção, $\Lambda^{0}(\mathfrak{g})=K, \Lambda^{1}(\mathfrak{g})=$ $\mathfrak{g}, V_{0}(\mathfrak{g})=U(\mathfrak{g})$ e $V_{1}(\mathfrak{g})=U(\mathfrak{g}) \otimes_{K} \mathfrak{g}$. Definimos o morfismo de $k$-álgebras $\varepsilon: V_{0}(\mathfrak{g})=U(\mathfrak{g}) \longrightarrow$ $K$ a projeção sobre $K$, isto é $\left.\varepsilon\right|_{K}=i d_{K}$ e $\varepsilon(\mathfrak{g})=0$, e

$$
\begin{gathered}
d_{1}: U(\mathfrak{g}) \otimes_{K} \mathfrak{g} \longrightarrow U(\mathfrak{g}) \\
u \otimes x \longmapsto u x,
\end{gathered}
$$

e assim, temos a sequencia exata

$$
V_{1}(\mathfrak{g}) \stackrel{d_{1}}{\longrightarrow} V_{0}(\mathfrak{g}) \stackrel{\varepsilon}{\longrightarrow} K \longrightarrow 0 .
$$

Agora, para cada $n \geq 2$, definimos $d_{n}: V_{n}(\mathfrak{g}) \longrightarrow V_{n-1}(\mathfrak{g})$ dada pela formula $d_{n}\left(u \otimes x_{1} \wedge \ldots \wedge\right.$ $\left.x_{n}\right)=\theta_{1}+\theta_{2}$ onde

$$
\begin{aligned}
& \theta_{1}=\sum_{i=1}^{p}(-1)^{i+1} u x_{i} \otimes x_{1} \wedge \ldots \wedge \hat{x}_{i} \wedge \ldots \wedge x_{p} \\
& \theta_{2}=\sum_{i<j}(-1)^{i+j} u \otimes\left[x_{i}, x_{j}\right] \wedge x_{1} \wedge \ldots \wedge \hat{x}_{i} \wedge \ldots \wedge \hat{x}_{j} \wedge \ldots \wedge x_{p} .
\end{aligned}
$$

para cada $u \in U(\mathfrak{g})$ e $g_{i} \in \mathfrak{g}$. Para o caso $n=2$, temos que

$$
d_{2}(u \otimes x \wedge y)=u x \otimes y-u y \otimes x-u \otimes[x, y]
$$

o complexo

$$
V_{\bullet}(\mathfrak{g}) \stackrel{\varepsilon}{\longrightarrow} K: \ldots \longrightarrow V_{1}(\mathfrak{g}) \stackrel{d_{1}}{\longrightarrow} V_{0}(\mathfrak{g}) \stackrel{\varepsilon}{\longrightarrow} K \longrightarrow 0
$$


é dito de complexo de Chevalley-Eilenberg, às vezes também é dito de complexo estândar. O Koszul mostrou que o complexo $V_{\bullet}(\mathfrak{g}) \stackrel{\varepsilon}{\longrightarrow} K$ é exato e assim temos uma resolução livre de $K$ por $U(\mathfrak{g})$-módulos. Para mais detalhes veja-se [(WEIBEL, 1994), Secção 7.7].

Definição 36. Uma resolução injetiva de um $R$-modulo $M$ é um complexo de $R$-módulos

$$
E^{\bullet}: 0 \longrightarrow E^{0} \stackrel{d^{0}}{\longrightarrow} E^{1} \stackrel{d^{1}}{\longrightarrow} E^{2} \longrightarrow \ldots
$$

onde cada $E^{i}$ é um $R$-modulo projetivo e existe um $R$-homomorfismo $\delta$ tais que o complexo aumentado

$$
M \rightarrow E^{\bullet}: 0 \longrightarrow M \stackrel{\delta}{\longrightarrow} E^{0} \stackrel{d^{0}}{\longrightarrow} E^{1} \stackrel{d^{1}}{\longrightarrow} E^{2} \longrightarrow \ldots
$$

é exato, isto é, é uma sequência exata.

\subsection{1 functor Tor e Ext}

Definição 37 (functor Tor ). Sejam $R$ um anel, $M, N R$-módulos e $P_{\bullet}$ uma resolução projetiva de $M$, isto é

$$
P_{\bullet}: \ldots \longrightarrow P_{2} \stackrel{d_{2}}{\longrightarrow} P_{1} \stackrel{d_{1}}{\longrightarrow} P_{0} \longrightarrow 0,
$$

aplicamos o functor covariante $-\otimes_{R} N$ ao complexo $P_{\bullet}$, e temos o complexo

$$
P_{\bullet} \otimes_{R} N: \ldots \longrightarrow P_{2} \otimes_{R} N \stackrel{d_{2} \otimes I d_{N}}{\longrightarrow} P_{1} \otimes_{R} N \stackrel{d_{1} \otimes I d_{N}}{\longrightarrow} P_{0} \otimes_{R} N \longrightarrow 0
$$

e assim podemos definir o functor Tor dado por

$$
\operatorname{Tor}_{n}^{R}(M, N):=H_{n}\left(P \bullet \otimes_{R} N\right)
$$

Na língua da Teoria das categorias, o functor Tor é o functor derivado do functor $-\otimes_{R} N$

Definição 38 (functor Ext ). Sejam $R$ um anel, $M, N R$-módulos e Seja $P_{\bullet}$ uma resolução projetiva de $M$, isto é

$$
P_{\bullet}: \ldots \longrightarrow P_{2} \stackrel{d_{2}}{\longrightarrow} P_{1} \stackrel{d_{1}}{\longrightarrow} P_{0} \longrightarrow 0
$$

aplicamos o functor contravariante $\operatorname{Hom}(-, N)$ ao complexo $P_{\bullet}$, e temos o complexo

$$
\operatorname{Hom}\left(P_{\bullet}, N\right): 0 \longrightarrow \operatorname{Hom}\left(P^{0}, N\right) \stackrel{d_{0}^{*}}{\longrightarrow} \operatorname{Hom}\left(P^{1}, N\right) \stackrel{d_{1}^{*}}{\longrightarrow} \operatorname{Hom}\left(P^{2}, N\right) \longrightarrow \ldots,
$$

e assim podemos definir o functor Ext dado por

$$
\operatorname{Ext}_{R}^{n}(M, N):=H^{n}\left(\operatorname{Hom}\left(P_{\bullet}, N\right)\right)
$$

Na linguá da teoria das categorias, o functor Ext é o functor derivado do functor $\operatorname{Hom}(-, N)$ 


\subsection{Homologia e Cohomologia de grupo}

\subsubsection{G-módulos}

Seja $G$ um grupo, denotaremos por $\mathbb{Z} G$ ao grupo abeliano livre gerado por $G$. As operações de $\mathbb{Z}$ e do $G$ induzem de maneira natural uma soma e um produto em $\mathbb{Z} G$, munindo à $\mathbb{Z} G$ de uma estrutura de anel, dito de o anel do grupo $G$ com coeficientes em $\mathbb{Z}$. Cada elemento $\alpha \in \mathbb{Z} G$ tem uma única expressão da forma $\sum_{g \in G} n_{g} g$ onde $n_{g} \in \mathbb{Z}$ para todo elemento $g \in G$, e $n_{g}=0$ exceto um numero finito de elementos $g \in G$. Isto é,

$$
\mathbb{Z} G=\left\{\sum_{g \in G} n_{g} g: n_{g} \in \mathbb{Z} \text { e } n_{g}=0 \text { exceto um numero finito de elementos de } G\right\}
$$

a soma e o produto em $\mathbb{Z} G$ estão dadas pelas seguintes formulas:

$$
\begin{aligned}
\sum_{g \in G} n_{g} g+\sum_{g \in G} m_{g} g & :=\sum_{g \in G}\left(n_{g}+m_{g}\right) g, \\
\left(\sum_{g \in G} n_{g} g\right) \cdot\left(\sum_{g \in G} m_{g} g\right) & :=\sum_{g \in G}\left(\sum_{h k=g} n_{h} \cdot m_{k}\right) g .
\end{aligned}
$$

Definição 39. Seja $G$ um grupo. Um $G$-modulo à esquerda é um grupo abeliano $A$ munido de uma ação de grupo $\rho: G \times A \longrightarrow A$, isto é:

(1) $\rho(e, a)=a$ para todo $a \in A$ (onde $e$ é o elemento neutro de $G$ ),

(2) $\rho(g, \rho(h, a))=\rho(g h, a)$, para todo $g, h \in G$ e para todo $a \in A$,

(3) $\rho\left(g, a_{1}+a_{2}\right)=\rho\left(g, a_{1}\right)+\rho\left(g, a_{2}\right)$, para todo $g \in G$ e para todo $a_{1}, a_{2} \in A$.

Denotaremos $\rho(g, a)$ por $g \cdot a$. Dizemos que um $G$-modulo $A$ é trivial se $G$ age trivialmente sobre $A$, isto é $g \cdot a=a$ para todo $g \in G$ e $a \in A$. Para nosso proposito nos consideraremos $\mathbb{Z}$ como um $G$-modulo trivial. Entenderemos um $G$-modulo como um $\mathbb{Z} G$-modulo, onde a ação de $G$ sobre $A$ é o produto escalar do $\mathbb{Z} G$-modulo restrito a $G$.

Definição 40. Seja $A$ um $G$-modulo, denotamos por $A^{G}$ o subgrupo $G$-invariantes, formado pelos elementos $a \in A$ tais que $g \cdot a=a$, para todo $g \in G$, isto é

$$
A^{G}:=\{a \in G: g \cdot a=a, \forall g \in G\} .
$$

Definição 41. Sejam $A, B$ dois $G$-módulos, um homomorfismo de grupos

$$
f: A \longrightarrow B
$$

é dito de $G$-morfismo se,

$$
f(g \cdot a)=g \cdot f(a), \forall g \in G, \forall a \in A .
$$


Além disso, os $G$-morfismos pode-se considerar homomorfismos de $\mathbb{Z} G$-módulos.Denotaremos por $\operatorname{hom}_{G}(A, B)$ o conjunto dos $G$-morfismos de $A$ em $B$. Se existe-se um $G$-isomorfismo (isto é, um $G$-morfismo bijectivo cuja inversa é também um $G$-morfismo), dizemos que $A$ e $B$ são isomorfos e que as ações de $G$ em $A$ e $B$ são equivalentes.

Exemplo 5 (Exemplo de $G$-modulo). Sejam $G$ e $H$ grupos abelianos. Para cada $n \geq 0$, denotamos por $\mathscr{F}\left(G^{n+1}, H\right)$ o grupo formado por todas as aplicações de $G^{n+1} \longrightarrow H$, podemos munir ao $\mathscr{F}\left(G^{n+1}, H\right)$ uma estrutura de $G$-modulo com ação à esquerda definida por

$$
(g \cdot f)\left(g_{0}, \ldots, g_{n}\right):=f\left(g^{-1} g_{0}, \ldots, g^{-1} g_{n}\right) .
$$

Assim, o subgrupos dos elementos $G$-invariantes, para cada $n \geq 1$, é dado por $\mathscr{F}\left(G^{n+1}, H\right)^{G}=\left\{f \in \mathscr{F}\left(G^{n+1}, H\right): f\left(g^{-1} g_{0}, \ldots, g^{-1} g_{n}\right)=f\left(g_{0}, \ldots, g_{n}\right), \quad \forall g, g_{0}, \ldots, g_{n} \in G\right\}$. Podemos munir ao $\mathscr{F}\left(G^{n+1}, H\right)$ de outra estrutura de $G$-modulo, a dizer

$$
(g \cdot f)\left(g_{0}, \ldots, g_{n}\right):=f\left(g_{0} g, \ldots, g_{n} g\right) .
$$

Tais estruturas para $\mathscr{F}\left(G^{n+1}, H\right)$ são equivalentes, isto é

$$
\begin{aligned}
\varphi: \mathscr{F}\left(G^{n+1}, H\right) & \longrightarrow \mathscr{F}\left(G^{n+1}, H\right) \\
f & \longmapsto \bar{f}
\end{aligned}
$$

onde $\bar{f}\left(g_{0}, \ldots, g_{n}\right)=f\left(g_{0}^{-1}, \ldots, g_{n}^{-1}\right), g_{0}, g_{1}, \ldots, g_{n} \in G$. Se $H$ é um $G$-modulo, podemos definir outra estrutura de $G$-modulo sobre $\mathscr{F}\left(G^{n+1}, H\right)$, dada por

$$
(g \cdot f)\left(g_{0}, \ldots, g_{n}\right):=g \cdot f\left(g^{-1} g_{0}, \ldots, g^{-1} g_{n}\right) .
$$

Exemplo 6 (Resolução injetiva para um $G$-modulo). Seja $A$ um $G$-modulo, consideremos a sequência

$$
0 \longrightarrow A \stackrel{\varepsilon}{\longrightarrow} \mathscr{F}(G, A) \stackrel{d^{0}}{\longrightarrow} \mathscr{F}\left(G^{2}, A\right) \stackrel{d^{1}}{\longrightarrow} \ldots
$$

onde $\mathscr{F}\left(G^{n+1}, A\right)$ tem a ação $(g . f)\left(g_{0}, \ldots, g_{n}\right):=g \cdot f\left(g^{-1}, g_{0}, \ldots, g^{-1} g_{n}\right)$ e

$$
\begin{aligned}
& \varepsilon: A \longrightarrow \mathscr{F}(G, H) \\
& a \longmapsto \varepsilon(a)
\end{aligned}
$$

definido por $\varepsilon(a)(g):=a$, para cada $g \in G$, com as diferencias dadas por

$$
\begin{aligned}
d^{n}: \mathscr{F}\left(G^{n+1}, A\right) & \longrightarrow \mathscr{F}\left(G^{n+2}, H\right) \\
f & \longmapsto d^{n}(f)
\end{aligned}
$$

definido por

$$
\begin{aligned}
\varepsilon(e)\left(g_{0}\right) & =e \\
\left(d^{n} f\right)\left(g_{0}, \ldots, g_{n+1}\right) & =f\left(1, g_{0}, \ldots, \hat{g}_{i}, \ldots, g_{n+1}\right) .
\end{aligned}
$$


(o símbolo ^ significa que omitimos essa variável) A sequencia acima é uma resolução injetiva de $A$ a qual admite uma homotopia contratante $\left(s^{n}\right)$ definida por

$$
\left(s^{n} f\right)\left(g_{0}, \ldots, g_{n-1}\right)=f\left(1, g_{0}, \ldots, g_{n-1}\right)
$$

A resolução anterior é chamada Resolução estândar de $A$. Sempre que $A$ seja um $G$-modulo, vamos a considerar o $G$-módulo $\mathscr{F}\left(G^{n+1}, A\right)$ munido com a ação

$$
(g . f)\left(g_{0}, \ldots, g_{n}\right)=g \cdot f\left(g^{-1}, g_{0}, \ldots, g^{-1} g_{n}\right),
$$

a menos que se indique o contrario.

\subsubsection{Homologia de grupo}

Definição 42. Dado um grupo $G$, definimos o homomorfismo aumentado de $G$ como sendo o homomorfismo de anéis

$$
\begin{aligned}
& \varepsilon: \mathbb{Z} G \longrightarrow \mathbb{Z} \\
& \sum_{g \in G} n_{g} g \longmapsto \sum_{g \in G} n_{g} .
\end{aligned}
$$

Denotaremos por $I_{G}$ ao núcleo do homomorfismo aumentado. Claramente $\{g-1: g \in G\}$ é um $\mathbb{Z}$-base para $I_{G}$, definimos o grupo de coinvariantes do $G$-modulo $A$ como o grupo abeliano

$$
A_{G}:=A / I_{G} A
$$

onde $I_{G} A$ é visto como o $\mathbb{Z} G$-submódulo de $A$, isto é, $I_{G} A$ é o $\mathbb{Z} G$-submódulo de $A$ gerado por $\{g . a-a: g \in G, a \in A\}$. Note-se que $A_{G}$ é o maior $G$-modulo quociente de $A$ onde $G$ age trivialmente.

Observação 15. Segue da definição que

$$
A_{G} \cong \mathbb{Z} \otimes_{\mathbb{Z} G} A \cong A \otimes_{\mathbb{Z} G} \mathbb{Z}
$$

de fato,

$$
A_{G}=A / I_{G} A \cong A \otimes_{\mathbb{Z} G} \mathbb{Z} G / I_{G} \cong A \otimes_{\mathbb{Z} G} \mathbb{Z}
$$

Assim, podemos definir o functor $-_{G}$ da categoria dos $G$-módulos (denotada por $\operatorname{Mod}_{G}$ ) na categoria dos grupos abelianos (denotada por Ab)como:

$$
\begin{aligned}
-_{G}: \operatorname{Mod}_{G} & \longrightarrow \mathrm{Ab} \\
A & \longmapsto A_{G}, \\
(f: A \rightarrow B) & \longmapsto\left(f_{G}: A_{G} \rightarrow B_{G}\right),
\end{aligned}
$$


onde $f_{G}: A_{G} \rightarrow B_{G}$ é tais que o diagrama

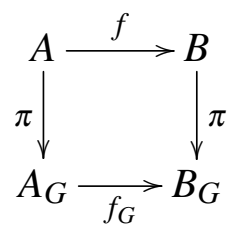

é comutativo, onde $\pi$ são as projeções. A boa definição de $f_{G}$ segue do fato

$$
f\left(I_{G} A\right) \subset I_{G} B
$$

Definição 43. Seja $G$ um grupo e $A$ um $G$-modulo. Definimos os grupos de homologia de grupo de $G$ com coeficientes em $A$ por

$$
H_{n}(G, A):=\operatorname{Tor}_{n}^{\mathbb{Z} G}(\mathbb{Z}, A)
$$

para todo $n \geq 0$.

Observação 16. Se $A$ é um $G$-modulo, segue da definição que:

$$
H_{0}(G, A)=\operatorname{Tor}_{0}^{\mathbb{Z} G}(\mathbb{Z}, A)=\mathbb{Z} \otimes_{\mathbb{Z} G} A=A_{G}
$$

No caso de $A$ ser um $G$-modulo projetivo, isto é, $A$ é um $\mathbb{Z} G$-modulo projetivo, temos:

$$
H_{n}(G, A) \cong\left\{\begin{array}{lll}
A_{G} & \text {, se } & n=0 \\
0 & \text {, se } & n \neq 0 .
\end{array}\right.
$$

Exemplo 7. Seja $G=\{1\}$, então $\mathbb{Z} G=\mathbb{Z}$ e quaisquer $G$-modulo $A$ é trivial, pois $1 . a=a$ para todo $a \in A$, e por tanto $A_{G}=A$. Além disso $\mathbb{Z}$ é um $\mathbb{Z} G$-modulo livre, então

$$
H_{n}(G, A) \cong\left\{\begin{array}{lll}
A & \text {, se } & n=0 \\
0 & \text {, se } & n \neq 0
\end{array}\right.
$$

\subsubsection{Cohomologia de grupo}

Definição 44. Sejam $G$ um grupo e $A$ um $G$-modulo, o $n$-ésimo grupo de cohomologia de $G$ com coeficientes em $A$ o qual denotamos por $H^{n}(G, A)$ é definido por

$$
H^{n}(G, A):=\operatorname{Ext}_{\mathbb{Z} G}^{n}(\mathbb{Z}, A),
$$

onde $\mathbb{Z}$ é o $G$-modulo trivial.

Se iniciamos com uma resolução projetiva de $\mathbb{Z}$ por $\mathbb{Z} G$-módulos

$$
P_{\bullet} \longrightarrow \mathbb{Z}: \ldots \longrightarrow P_{2} \longrightarrow P_{1} \longrightarrow P_{0} \longrightarrow \mathbb{Z} \longrightarrow 0
$$

logo aplicando o functor contravariante $\operatorname{Hom}_{\mathbb{Z} G}(-, A)$ e calculamos a cohomologia do complexo resultante, obtemos a cohomologia do grupo

$$
H^{n}(G, A)=H^{n}\left(\operatorname{Hom}_{\mathbb{Z} G}\left(P_{\bullet}, A\right)\right) .
$$


Observação 17. A Homologia e Cohomologia de grupos independem da escolha da resolução.

Exemplo 8. Seja $B_{0}(G)$ o $\mathbb{Z} G$-módulo gerado pelo simbolo [ ] o qual é isomorfo a $\mathbb{Z} G$ e assim, podemos identificar [ ] com $1 \in \mathbb{Z} G$. Para cada $n \geq 1$, seja $B_{n}(G)$ o $\mathbb{Z} G$-módulo livre gerado pelos símbolos $\left[g_{1}|\ldots| g_{n}\right] \operatorname{com} g_{i} \in G$. Seja $\varepsilon: \mathbb{Z} G \longrightarrow \mathbb{Z}$ definido como antes, e definimos para cada $n \geq 1$, as diferencias $d_{n}: B_{n}(G) \longrightarrow B_{n-1}(G)$ dada por $d_{n}=\sum_{i=0}^{n}(-1)^{i} d_{n}^{i}$, onde

$$
\begin{aligned}
& d_{n}^{0}\left(\left[g_{1}|\ldots| g_{n}\right]\right)=g_{1}\left[g_{2}|\ldots| g_{n}\right] ; \\
& d_{n}^{i}\left(\left[g_{1}|\ldots| g_{n}\right]\right)=\left[g_{1}|\ldots| g_{i} g_{i+1}|\ldots| g_{n}\right] \text { para } i=1, \ldots, n-1 ; \\
& d_{n}^{n}\left(\left[g_{1}|\ldots| g_{n}\right]\right)=\left[g_{1}|\ldots| g_{n-1}\right] .
\end{aligned}
$$

e assim temos uma resolução livre de $\mathbb{Z}$, a qual é dita de Resolução Bar

$$
B(G) \bullet \longrightarrow \mathbb{Z}: \ldots \longrightarrow B_{n}(G) \stackrel{d_{n}}{\longrightarrow} B_{n-1}(G) \ldots \longrightarrow B_{2}(G) \stackrel{d_{2}}{\longrightarrow} B_{1}(G) \stackrel{d_{1}}{\longrightarrow} B_{0}(G) \stackrel{\varepsilon}{\longrightarrow} \mathbb{Z} \longrightarrow 0
$$

Exemplo 9. Sejam $G$ um grupo e $G^{n}$ o produto cartesiano de $n$ copias de $G$. Seja $C_{n}(G):=G^{n+1}$ com ação dada por

$$
g\left(g_{0}, g_{1}, \ldots, g_{n}\right):=\left(g g_{0}, g g_{1}, \ldots, g g_{n}\right) .
$$

$C_{n}(G)$ é um $G$-modulo livre com base $\left\{\left(1, g_{1}, \ldots, g_{n}\right): g_{i} \in G\right\}$ e assim, para $C_{0}(G)$ temos que é isomorfo ao $G$-modulo $\mathbb{Z} G$. Para cada $n \geq 1$, definimos $\delta_{n}: C_{n}(G) \longrightarrow C_{n-1}(G)$ dada por

$$
\delta_{n}\left(g_{0}, \ldots, g_{n}\right):=\sum_{i=0}^{n}(-1)^{i}\left(g_{0}, \ldots, \hat{g}_{i}, \ldots, g_{n}\right),
$$

onde $g_{i} \in G$. Assim temos uma resolução livre de $G$-módulos sobre $\mathbb{Z}$.

$$
C(G) \bullet \longrightarrow \mathbb{Z}: \ldots \longrightarrow C_{n}(G) \stackrel{\delta_{n}}{\longrightarrow} C_{n-1}(G) \ldots \longrightarrow C_{2}(G) \stackrel{\delta_{2}}{\longrightarrow} C_{1}(G) \stackrel{\delta_{1}}{\longrightarrow} C_{0}(G) \stackrel{\varepsilon}{\longrightarrow} \mathbb{Z} \longrightarrow 0
$$

Para cálculos, tomamos a resolução de $G$ dada no Exemplo 9 e aplicando o functor $\operatorname{Hom}_{\mathbb{Z} G}(-, A)$ obtemos

$$
C^{n}(G, A)=\operatorname{Hom}_{G}\left(C_{n+1}(G), A\right),
$$

é um $G$-modulo, com ação dada por $g . f\left(g_{0}, \ldots, g_{n}\right):=f\left(g g_{0}, \ldots, g g_{n}\right)$. As diferencias são dadas por

$$
\begin{aligned}
d f\left(g_{0}, \ldots, g_{n}\right)= & g_{0} \cdot f\left(g_{1}, \ldots, g_{n}\right)+\sum_{0 \leq i \leq n}(-1)^{i+1} f\left(g_{0}, \ldots, g_{i} g_{i+1}, \ldots, g_{n}\right)+ \\
& (-1)^{n+1} f\left(g_{0}, \ldots, g_{n-1}\right) .
\end{aligned}
$$

E assim, nos temos o complexo de cocadeias aumentado

$$
0 \longrightarrow A \stackrel{\varepsilon^{*}}{\longrightarrow} C^{1}(G, A) \stackrel{d^{1}}{\longrightarrow} C^{2}(G, A) \stackrel{d^{2}}{\longrightarrow} C^{3}(G, A) \stackrel{d^{3}}{\longrightarrow} \ldots
$$


onde $d^{n}=\delta_{n}^{*}$ a aumentação é dada por

$$
\begin{aligned}
\varepsilon^{*}: A & \rightarrow C^{1}(G, A) \\
a & \mapsto \varepsilon^{*}(a) .
\end{aligned}
$$

e $\varepsilon^{*}(a) g=g . a$, para cada $g \in G$. Por tanto

$$
H^{n}(G, A)=H^{n}\left(C^{\bullet}(G, A)\right)
$$

Observação 18. Similarmente à cohomologia de grupo, podemos definir outras teorias de cohomologias como segue:

(i) Se $G$ é um grupo topológico e $V$ um $G$-modulo com ação; $\rho: G \longrightarrow G L(V)$ contínua. Tomando o complexo de cadeias aumentada com diferencias como em 2.21 e com cocadeias $C^{n}(G, V)$ substituído por mapas contínuos denotados por $C_{\text {cont }}^{n}(G, V)$, a cohomologia do complexo resultante

$$
H_{\text {cont }}^{n}(G, V):=H^{n}\left(C_{\text {cont }}^{\bullet}(G, V)\right)
$$

é dita de cohomologia contínua.

(ii) Analogamente, se $G$ é um grupo de Lie e $V$ um $G$-modulo com ação $\rho: G \longrightarrow G L(V)$ suave $\left(C^{\infty}\right)$. Tomando o complexo de cadeias aumentada como em 2.21 e com cocadeias $C^{n}(G, V)$ substituídos por mapas suaves $\left(C^{\infty}\right)$ denotados por $C_{d}^{n}(G, V)$, a cohomologia do complexo resultante

$$
H_{d}^{n}(G, V):=H^{n}\left(C_{d}^{\bullet}(G, V)\right)
$$

é dita de cohomologia diferenciavel.

Proposição 13. Sejam $G$ um grupo de Lie conexo, $U$ um subgrupo compacto maximal e $X=G / U$. Seja $\mathfrak{g}$ e $\mathfrak{u}$ as álgebras de Lie de $G$ e $U$. Então

\subsection{Cohomologia de De Rham}

Seja $M$ uma variedade suave (de classe $C^{\infty}$ ). Para cada $p \geq 1$ denotamos por $\Omega^{p}(M)$ o espaço das $p$-formas diferenciáveis sobre $M$ e $\Omega^{0}(M)$ o espaço de funções suaves sobre $M$. Eles formam uma $\mathbb{R}$-álgebra graduada com produto dado pelo produto exterior $\wedge$ :

$$
\Omega^{\bullet}(M):=\bigoplus_{p \geq 0} \Omega^{p}(M)
$$

Além disso, nos temos as diferencias de De Rham (também ditas de derivadas exteriores) definida como o único mapa $\mathbb{R}$-linear $d: \Omega^{\bullet}(M) \longrightarrow \Omega^{\bullet+1}(M)$ tais que satisfazem as seguintes propriedades:

1. $d f=$ diferencial de $f$ para funções suaves, 
2. $d(d f)=0$

3. $d(\omega \wedge \eta)=(d \omega) \wedge \eta+(-1)^{p} \omega \wedge(d \eta)$ para $\omega \in \Omega^{p}(M)$ e $\eta \in \Omega^{q}(M)$.

O complexo de De Rham é o complexo de cocadeias das formas diferenciáveis sobre $M$, com as derivadas exteriores como as diferenciais:

$$
\Omega^{\bullet}(M): 0 \stackrel{d}{\longrightarrow} \Omega^{0}(M) \stackrel{d}{\longrightarrow} \Omega^{1}(M) \stackrel{d}{\longrightarrow} \Omega^{2}(M) \stackrel{d}{\longrightarrow} \ldots
$$

e assim definimos a cohomologia de De Rham

$$
H_{D R}^{n}(M):=H^{n}\left(\Omega^{\bullet}(M)\right) .
$$

Dado um campo vetorial $X$ sobre $M$, podemos definir o operador contração $i_{X}: \Omega^{\bullet}(M) \longrightarrow$ $\Omega^{\bullet-1}(M)$, verificando as seguintes propriedades

1. $i_{X} \theta=\theta(X)$, para todo $\theta \in \Omega^{1}(M)$,

2. $i_{X}(\omega \wedge \eta)=i_{X}(\omega) \wedge \eta+(-1)^{p} \omega \wedge i_{X}(\eta)$, onde $\omega \in \Omega^{p}(M)$ e $\eta \in \Omega^{q}(M)$.

Também existe um operador $\mathscr{L}_{X}: \Omega^{\bullet}(M) \longrightarrow \Omega^{\bullet}(M)$ dito de derivada de Lie, definido como o único mapa $\mathbb{R}$-linear tais que:

1. $\mathscr{L}_{X}(f)=X(f)$ para toda função suave,

2. $\mathscr{L}_{X} Y=[X, Y]$ onde $[$, ] é o colchete de Lie.

Temos a identidade $\mathscr{L}_{X}=i_{x} \circ d+d \circ i_{X}$ a qual é dita de formula magica de Cartan, que relaciona a derivada exterior $d$ e o operador contração $i_{X}$. Agora nos podemos expressar a diferencial de uma $p$-forma diferenciável em termos do colchete de Lie, a dizer

$$
\begin{aligned}
d \omega\left(X_{0} \wedge \ldots \wedge X_{p}\right)= & \sum_{0 \leq i<j \leq p}(-1)^{i+j} \omega\left(\left[X_{i}, X_{j}\right] \wedge X_{0} \wedge \ldots \wedge \hat{X}_{i} \ldots \wedge \hat{X}_{j} \wedge \ldots \wedge X_{p}\right)+ \\
& \sum_{0 \leq i \leq p}(-1)^{i} X_{i} . \omega\left(X_{0} \wedge \ldots \wedge \hat{X}_{i} \wedge \ldots \wedge X_{p}\right),
\end{aligned}
$$

para mais detalhes veja-se [(WARNER, 1983), Proposição 2.25].

Definição 45. Sejam $M$ uma variedade diferenciável, $G$ um grupo de Lie e $T: G \times M \longrightarrow M$ uma ação à esquerda de $G$ sobre $M$. Dizemos que uma $p$-forma $\omega \in \Omega^{p}(M)$ é dita de invariante à esquerda se $T_{g}^{*} \omega=\omega$, para todo $g \in G$. As formas invariantes à esquerda formam uma subálgebra o qual denotamos por $\Omega^{\bullet}(M)_{L}$.

Teorema 10. Sejam $M$ uma variedade diferenciável, $G$ um grupo de Lie compacto e $T: G \times$ $M \longrightarrow M$ uma ação à esquerda de $M$, então

$$
\mathrm{i}_{*}: H^{*}\left(\Omega^{\bullet}(M)_{L}\right) \longrightarrow H_{D R}^{*}(M)
$$

induzido pela inclusão é injetora. Além disso, se $G$ é conexo temos que $\mathrm{i}_{*}$ é um isomorfismo 


\subsection{Teorias de Homologia e Cohomologia}

Nesta secção daremos a definição da homologia e cohomologia singular,alem disso daremos as principais relações entre as teorias de homologia e cohomologia definidas.

\subsubsection{Homologia e Cohomologia singular}

Para cada $n \geq 0$, denotamos $\Delta^{n}$ o $n$-simplexo estândar ao conjunto

$$
\Delta^{n}:=\left\{\left(x_{0}, \ldots, x_{n}\right) \in \mathbb{R}^{n+1}: \sum_{i=0}^{n} x_{i} \leq 1,0 \leq x_{i}\right\}
$$

$\Delta^{0}$ é reduzido a um ponto denotado por 0 . Definimos as aplicações $\delta_{i}^{n}: \Delta^{n} \longrightarrow \Delta^{n+1}, i=$ $0, \ldots, n+1$ da seguinte forma

$$
\begin{array}{rc}
\delta_{0}^{0}(0) & =1 \\
\delta_{1}^{0}(0) & =0 \\
\delta_{0}^{n}\left(x_{1}, \ldots, x_{n}\right) & =\left(1-x_{1}-\ldots-x_{n}, x_{1}, x_{2}, \ldots, x_{n}\right) \\
\delta_{i}^{n}\left(x_{1}, \ldots, x_{n}\right) & =\left(x_{1}, \ldots, x_{i-1}, 0, x_{i}, \ldots, x_{n}\right)
\end{array}
$$

Seja $X$ um espaço topológico. Um $n$-simplexo singular de $X$ é uma aplicação contínua $\sigma: \Delta^{n} \longrightarrow X$, denotamos $R_{n}(X)$ o conjunto de tais aplicações, e denotamos por $S_{n}(X)$ o $\mathbb{Z}$-modulo livre gerado por $R_{n}(X)$. Em particular $R_{0}(X)=X$ e $S_{0}(X)$ o $\mathbb{Z}$-modulo livre gerado por $X$, os elementos de $S_{n}(X)$ são chamados $n$-cadeias singulares. As faces de um $n$-simplexo singular $\sigma$ são as aplicações $\sigma^{i}=\sigma \circ \delta_{i}^{n-1}, i=0, \ldots, n$, o bordo de $\sigma$ é o elemento

$$
\partial_{n} \sigma=\sum_{i=0}^{n+1}(-1)^{i} \sigma^{i} \in S_{n-1}(X) .
$$

Assim temos o complexo

$$
S_{\bullet} \rightarrow \mathbb{Z}: \ldots \stackrel{\partial_{2}}{\longrightarrow} S_{1}(X) \stackrel{\partial_{1}}{\longrightarrow} S_{0}(X) \stackrel{\varepsilon}{\longrightarrow} \mathbb{Z} \longrightarrow 0
$$

onde

$$
\varepsilon\left(\sum n_{x} x\right)=\sum n_{x}
$$

para cada $\sum n_{x} x \in S_{0}(X)$. Agora, seja $G$ um grupo abeliano, aplicando o functor $-\otimes_{\mathbb{Z}} G$ e $\operatorname{Hom}(-, G)$ ao complexo (2.31), obtemos dois complexos

$$
\begin{gathered}
S_{\bullet}(X) \otimes_{\mathbb{Z}} G: \ldots \stackrel{\partial_{2} \otimes \mathrm{id}_{G}}{\longrightarrow} S_{1}(X) \otimes_{\mathbb{Z}} G \stackrel{\partial_{1} \otimes \mathrm{id}_{G}}{\longrightarrow} S_{0}(X) \otimes_{\mathbb{Z}} G \longrightarrow 0, \\
\operatorname{Hom}\left(S_{\bullet}(X), G\right): 0 \longrightarrow \operatorname{Hom}\left(S_{0}(X), G\right) \stackrel{\partial_{1}^{*}}{\longrightarrow} \operatorname{Hom}\left(S_{1}(X), G\right) \stackrel{\partial_{2}^{*}}{\longrightarrow} \ldots
\end{gathered}
$$

Denotamos por $H_{n}(X, G)$ à homologia do complexo $S_{\bullet}(X) \otimes_{\mathbb{Z}} G$, isto é

$$
H_{n}(X, G):=H_{n}\left(S_{\bullet}(X) \otimes_{\mathbb{Z}} G\right),
$$


a qual é dita de $n$-ésimo grupo de homologia singular do espaço $X$ com coeficientes em $G$. Analogamente, denotamos por $H^{n}(X, G)$ à cohomologia do complexo $\operatorname{Hom}\left(S_{\bullet}(X), G\right)$, isto é

$$
H^{n}(X, G):=H^{n}\left(\operatorname{Hom}_{\mathbb{Z}}\left(S_{\bullet}(X), G\right)\right) .
$$

a qual é dita de $n$-ésimo grupo de cohomologia singular do espaço $X$ com coeficientes em $G$.

Teorema 11 (Teorema de De Rham). Seja $M$ uma variedade e seja o homomorfismo

$$
\begin{aligned}
I_{n}: H_{D R}^{n}(M) & \longrightarrow \operatorname{Hom}\left(H_{n}(M ; \mathbb{R}), \mathbb{R}\right) \\
{[\omega] } & \longmapsto I_{n}([\omega]),
\end{aligned}
$$

onde

$$
\begin{aligned}
I([\omega]): H_{n}(M ; \mathbb{R}) & \longrightarrow \mathbb{R} \\
{[\sigma] } & \longmapsto \int_{\sigma} \omega,
\end{aligned}
$$

então $I_{n}$ é um isomorfismo para cada $n \geq 0$.

Demonstração. Vide [(WARNER, 1983), Teorema 5.36]

Proposição 14. A cohomologia do grupo de Lie clássico $S U / S O(\mathbb{R})$ é dada por

$$
H^{*}(S U / S O(\mathbb{R}) ; \mathbb{R}) \cong \Lambda\left(x_{5}, \ldots, x_{4 k+1}, \ldots\right)
$$

onde os geradores são indecomponíveis.

Demonstração. Vide [(MIMURA; TODA, 1991), Capitulo IV 3.16]

\subsubsection{Homotopia racional dos $\mathrm{H}$-espaços}

Definição 46. Seja $(X, e)$ um espaço topológico com um ponto base. Dizemos que $X$ é um $H$-espaço se existe um mapa continuo $\mu: X \times X \rightarrow X$, tais que, $\mu(x, e)=\mu(e, x)=x$, para todo $x \in X$. Dizemos que um $H$-espaço é associativo se os mapas $\mu \circ(i d \times \mu), \mu \circ(\mu \times i d)$ : $X \times X \times X \rightarrow X$ são homotopicamente equivalentes.

Observação 19. Todo grupo topológico é um $H$-espaço associativo, em particular um grupo de Lie é um $H$-espaço associativo.

Proposição 15. Sejam $(X, e)$ um $H$-espaço conexo por caminhos associativo e $K$ um corpo. Então, $H_{*}(X):=\bigoplus_{i=0}^{\infty} H_{i}(X)$ tem uma estrutura de álgebra de Hopf conexa com coproduto comutativo induzido pelo mapa diagonal $\Delta: X \longrightarrow X \times X$.

Demonstração. Vide [(ROTMAN, 1988), Teorema 12.42]

O Teorema 7 tem um corolário geométrico relacionando a homologia e homotopia dos $H$-espaços. 


\subsubsection{Produto Whitehead}

Dado $(X, e)$ um $H$-espaço conexo consideremos $\pi_{n}(X)$ o $n$-ésimo grupo de homotopia com ponto base $e$, se define um morfismo $[\cdot, \cdot]: \pi_{n+1}(X) \otimes_{\mathbb{Z}} \pi_{m+1}(X) \longrightarrow \pi_{n+m+1}(X)$ (para detalhes da construção ver (SAMELSON, 1953)). Daremos um bosquejo da construção. O produto $S^{m} \times S^{n}$ de duas esferas tem uma $C W$-descomposição em quatro células, de dimensões 0 , $m, n$ e $m+n$ (a definição de $C W$-complexo sera dada no Capitulo 3). A a união das três primeiras células é $S^{m} \vee S^{n}$. O mapa de colagem $S^{m+n-1} \longrightarrow S^{m} \vee S^{n}$, é dito o mapa de Whitehead.

A seguinte construção descreve o mapa de Whitehead a menos de homotopia. Existe uma canônica descrição do mapa $w: S^{m+n-1} \longrightarrow S^{m} \vee S^{n}$. A esfera $S^{m+n-1}$ é a união dos seguintes conjuntos fechados

$$
\begin{aligned}
& U=\left\{\left(x_{1}, \ldots, x_{m+n}\right) \in S^{m+n-1}: x_{1}^{2}+\ldots+x_{m+n}^{2} \leq \frac{1}{2}\right\} \\
& V=\left\{\left(x_{1}, \ldots, x_{m+n}\right) \in S^{m+n-1}: x_{1}^{2}+\ldots+x_{m+n}^{2} \geq \frac{1}{2}\right\}
\end{aligned}
$$

e assim temos que $U \simeq D^{m} \times S^{n-1}, V \simeq S^{m-1} \times D^{n}$ e $U \cap V=S^{m-1} \times S^{n-1}$. A descomposição $S^{m+n-1}=U \cup V$ também pode-se construir da seguinte maneira

$$
\left.S^{m+n-1}=\partial D^{m+n} \simeq \partial\left(D^{m} \times D^{n}\right)=\left(D^{m} \times \partial D^{n}\right) \cup\left(\partial D^{m} \times D^{n}\right)=D^{m} \times S^{n}\right) \cup\left(S^{m} \times D^{n}\right)
$$

e assim nosso mapa $w: S^{m+n-1} \longrightarrow S^{m} \vee S^{n}$ consiste de duas projeções

$$
\begin{aligned}
& U=D^{m} \times S^{n-1} \longrightarrow D^{m} \longrightarrow D^{m} / S^{m-1}=S^{m} \subset S^{m} \vee S^{n}, \\
& V=S^{m-1} \times D^{n} \longrightarrow D^{n} \longrightarrow D^{n} / S^{n-1}=S^{n} \subset S^{m} \vee S^{n}
\end{aligned}
$$

tomando a "superfície de corte" $S^{m-1} \times S^{n-1}$ num ponto.

Agora sejam $f: S^{m} \longrightarrow X, g: S^{n} \longrightarrow X$ dois esferoides de algum com ponto base $x_{0}$. Eles geram o mapa $S^{m} \vee S^{n} \longrightarrow X$, e a composição de este mapa com $w$ é um esferoide $r: S^{m+n-1} \longrightarrow X$. Pode-se mostrar que a classe de homotopia de $r$ se pode determinar pelas classes de homotopias das $f$ e $g$. Assim dado $\alpha=[f] \in \pi_{m}\left(X, x_{0}\right)$ e $\beta=[g] \in \pi_{n}\left(X, x_{0}\right)$, então definimos $[\alpha, \beta]:=[r]$ o qual é dito de Produto de Whitehead. O mapa

$$
[\cdot, \cdot]: \pi_{n+1}(X) \otimes_{\mathbb{Z}} \pi_{m+1}(X) \longrightarrow \pi_{n+m+1}(X)
$$

verifica as seguintes propriedades:

(1) Se $\alpha \in \pi_{n+1}(X)$ e $\beta \in \pi_{m+1}(X)$, então $[\alpha, \beta]=(-1)^{n m+1}[\beta, \alpha]$

(2) Se $\alpha \in \pi_{n+1}(X), \beta \in \pi_{m+1}(X), \gamma \in \pi_{r+1}(X)$, então

$$
(-1)^{n r}[\alpha,[\beta, \gamma]]+(-1)^{n m}[\beta,[\gamma, \alpha]]+(-1)^{n r}[\gamma,[\alpha, \beta]]=0
$$

(3) Se $\alpha \in \pi_{n+1}(X), \beta \in \pi_{m+1}(X)$, e $h: \pi_{*}(X) \longrightarrow H_{*}(X)$ é o mapa de Hurewicz, então

$$
h([\alpha, \beta])=h(\alpha) * h(\beta)-(-1)^{(n+1)(m+1)} h(\beta) * h(\alpha) .
$$


para detalhes de (1),(2) vide [(FOMENKO; FUCHS, 2016),Secção 10.5] e para (3) vide (SAMELSON, 1953).

Assim, considerando $L_{n}:=\pi_{n+1}(X, e)$ para todo $n \geq 0$ e por (1) e (2), temos que $L=$ $\bigoplus_{n=0}^{\infty} L_{n}$ é uma álgebra de Lie, e o homomorfismo de Hurewicz $h: \pi_{*}(X, e) \longrightarrow H_{*}(X)$ é um morfismo de álgebras de Lie de grau 1. Além disso, pela Proposição 15 e o Teorema dos coeficientes universais para Homologia temos que $H_{*}(X, \mathbb{Q})=H_{*}(X) \otimes_{\mathbb{Z}} \mathbb{Q}$ é um álgebra de Hopf conexa com coproduto comutativo. Definimos $P_{n}(X, \mathbb{Q}):=P_{n}\left(H_{*}(X, \mathbb{Q})\right)$ e pelo Teorema 7 temos que $U\left(P_{*}(X, Q)\right)=H_{*}(X, \mathbb{Q})$.

Teorema 12 (Cartan-Serre). Seja $(X, e)$ um $H$-espaço conexo por caminhos. Então o homomorfismo de Hurewicz racional é um isomorfismo de álgebras de Lie

$$
h_{\mathbb{Q}}: \pi_{*}(X, e) \otimes_{\mathbb{Z}} \mathbb{Q} \longrightarrow P_{*}(X, \mathbb{Q}) .
$$

Por tanto, obtemos um isomorfismo de álgebras de Hopf

$$
U\left(h_{\mathbb{Q}}\right): U\left(\pi_{*}(X, e) \otimes \mathbb{Q}\right) \longrightarrow H_{*}(X, \mathbb{Q}) .
$$

Demonstração. Vide [(BASS; PEDRINI, 1997), Teorema 5.2]

\subsubsection{Relações entre a homologia e cohomologia de grupos}

Sejam $X$ um espaço topológico e $G$ um grupo operando por homomorfismo sobre $X$, isto é $G$ age sobre $X$ e cada translação é contínua (consideramos a $G$ com a topologia discreta), denotamos $G / X$ o espaço de orbitas de $G$ em $X$ com a topologia quociente, e $\pi: X \longrightarrow G / X$ a aplicação quociente de $X$ em $X / G$. Definimos uma ação de $G$ em $R_{n}(X)$ por

$$
(g . \sigma)(t)=g . \sigma(t)
$$

e estendemos por linearidade a $S_{n}(X)$, e assim $S_{n}(X)$ é um $G$-modulo e

$$
S_{\bullet}(X) \stackrel{\eta}{\longrightarrow} \mathbb{Z}: \ldots \stackrel{\partial_{1}}{\longrightarrow} S_{1}(X) \stackrel{\partial_{0}}{\longrightarrow} S_{0}(X) \stackrel{\eta}{\longrightarrow} \mathbb{Z} \longrightarrow 0
$$

um complexo de $G$-módulos.

Lema 3. Se $X$ é um espaço topológico contrátil, o complexo

$$
S_{\bullet}(X) \stackrel{\eta}{\longrightarrow} \mathbb{Z}: \ldots \stackrel{\partial_{1}}{\longrightarrow} S_{1}(X) \stackrel{\partial_{0}}{\longrightarrow} S_{0}(X) \stackrel{\eta}{\longrightarrow} \mathbb{Z} \longrightarrow 0
$$

é exato, e portanto uma resolução projetiva de $\mathbb{Z}$.

Demonstração. Vide [(GUICHARDET, 1980), Lema 14.1]

Lema 4. Sejam $X$ um espaço topológico, $G$ um grupo que age livremente sobre $X$ e que $\pi: X \longrightarrow G / X$ é uma aplicação de recobrimento. Então o $G$-modulo $S_{n}(X)$ é relativamente projetivo e temos $S_{\bullet}(X)^{G} \sim S_{\bullet}(G / X)$ (isomorfismo de complexos) onde $S_{\bullet}(X)^{G}$ é o grupo dos elementos $G$-invariantes, na verdade $S_{n}(X)$ é um $\mathbb{Z} G$-modulo livre. 
Demonstração. Veja [(GUICHARDET, 1980), Lema 14.2]

Proposição 16. Seja $X$ é um espaço topológico contrátil, $G$ um grupo que age livre sobre $X$ e que $\pi: X \longrightarrow G / X$ é uma aplicação de recobrimento. Então

$$
\begin{gathered}
H_{n}(G, A) \cong H_{n}(G / X, A) \\
H^{n}(G, A) \cong H^{n}(G / X, A),
\end{gathered}
$$

onde $A$ é um $G$-modulo trivial.

Demonstração. Pelo Lema 3 o complexo

$$
S_{\bullet}(X) \stackrel{\eta}{\longrightarrow} \mathbb{Z}: \ldots \stackrel{\partial_{1}}{\longrightarrow} S_{1}(X) \stackrel{\partial_{0}}{\longrightarrow} S_{0}(X) \stackrel{\eta}{\longrightarrow} \mathbb{Z} \longrightarrow 0
$$

é uma resolução projetiva do $G$-modulo trivial $\mathbb{Z}$, então $H_{*}(G, A)$ e $H^{*}(G, A)$ são a homologia e cohomologia dos seguintes complexos

$$
\ldots \longrightarrow S_{1}(X) \otimes_{G} A \longrightarrow S_{0}(X) \otimes_{G} A \longrightarrow 0
$$

$\mathrm{e}$

$$
0 \longrightarrow \operatorname{Hom}_{G}\left(S_{0}(X), A\right) \longrightarrow \operatorname{Hom}_{G}\left(S_{1}(X), A\right) \longrightarrow \ldots
$$

e pelo Lema 4 temos

$$
S_{\bullet}(X) \otimes_{G} A \sim S_{\bullet}(X)^{G} \otimes_{\mathbb{Z}} A \sim S_{\bullet}(G / X) \otimes_{\mathbb{Z}} A,
$$

e assim

$$
H_{n}(G, A) \cong H_{n}(G / X, A),
$$

para cada $n \geq 0$. Analogamente, pelo Lema 4 e do fato que $-{ }^{G}$ é um functor adjunto á esquerda do functor $\operatorname{Hom}(\mathbb{Z},-)$ temos que

$$
\operatorname{Hom}_{G}\left(S_{\bullet}(X), A\right) \sim \operatorname{Hom}\left(S_{\bullet}(X)^{G}, A\right) \sim \operatorname{Hom}\left(S_{\bullet}(G / X), A\right)
$$

e assim temos

$$
H^{n}(G, A) \cong H^{n}(G / X, A) .
$$

\subsection{Cohomologia de Álgebra de Lie}

Nesta secção daremos a definição de uma álgebra de Lie com coeficientes num $\mathfrak{g}$-modulo $V$. Também daremos alguns isomorfismos com a cohomologia de De Rham. 


\subsubsection{Cohomologia de De Rham de um grupo de Lie}

Sejam $K$ um corpo e $\mathfrak{g}$ uma álgebra de Lie sobre $K$. Um $\mathfrak{g}$-modulo é um espaço vetorial $V$ sobre $K$ sobre o qual $\mathfrak{g}$ age via um homomorfismo $\rho: \mathfrak{g} \longrightarrow \mathfrak{g l}(V)$. Equivalentemente , um $\mathfrak{g}$-modulo pode ser visto como um modulo sobre o anel $U(\mathfrak{g})$. A ação correspondente de $x \in \mathfrak{g}$ sobre $v \in V$ será denotada por $x \cdot v$.

Definição 47. Seja $\mathfrak{g}$ uma álgebra de Lie sobre sobre um corpo $K$ e seja $V$ um $\mathfrak{g}$-modulo, o $n$-ésimo grupo de cohomologia de g com coeficientes em $V$ o qual é denotado por $H^{n}(\mathfrak{g}, V)$ é definido por

$$
H^{n}(\mathfrak{g}, V):=\operatorname{Ext}_{U(\mathfrak{g})}^{n}(K, V)
$$

Em particular, temos do Exemplo 4 uma resolução projetiva de $K$ por $U(\mathfrak{g})$-módulos a qual gera o complexo Chevalley-Eilenberg-Koszul com cocadeias dadas por

$$
C^{n}(\mathfrak{g} ; V):=\operatorname{Hom}_{U(\mathfrak{g})}\left(U(\mathfrak{g}) \otimes \Lambda^{n} \mathfrak{g}, V\right) \cong \operatorname{Hom}_{K}\left(\Lambda^{n} \mathfrak{g}, V\right)
$$

com as diferencias $d^{n}: C^{n}(g ; V) \longrightarrow C^{n+1}(g ; V)$ dadas por

$$
\begin{aligned}
\left(d^{n} f\right)\left(x_{0}, \ldots, x_{n}\right)= & \sum_{i=0}^{n}(-1)^{i} x_{i} . f\left(x_{0}, \ldots, \hat{x}_{i}, \ldots, x_{n}\right)+ \\
& \sum_{i<j}(-1)^{i+j} f\left(\left[x_{i}, x_{j}\right], x_{0}, \ldots, \hat{x}_{i}, \ldots, \hat{x}_{j}, \ldots, x_{n}\right),
\end{aligned}
$$

onde, como sempre, ^significa que o elemento está omitido. Assim $H^{*}(\mathfrak{g}, V)$ é dada pela cohomologia do complexo

$$
C^{\bullet}(\mathfrak{g} ; V): 0 \longrightarrow C^{0}(\mathfrak{g}, V) \stackrel{d^{0}}{\longrightarrow} C^{1}(\mathfrak{g} ; V) \stackrel{d^{1}}{\longrightarrow} C^{2}(\mathfrak{g} ; V) \stackrel{d^{2}}{\longrightarrow} \ldots
$$

e assim $H^{*}(\mathfrak{g}, V)=H^{*}\left(C^{\bullet}(\mathfrak{g} ; V)\right)$, assim podemos tomar isto como a definição da cohomologia de álgebra de Lie.

Daremos uma interpretação geométrica desta definição. Vemos que a equação (2.43) é a mesma que a equação (2.25), o qual mostra que o complexo para a cohomologia de álgebra de Lie realmente se origina do complexo de De Rham. Além disso, nos temos que gé a álgebra de Lie do grupo de Lie real conexo $G$.

Para cada elemento $g \in G$, sejam $l_{g}: G \longrightarrow G$ e $r_{g}: G \longrightarrow G$ as ações a esquerda e direita respectivamente, dadas por:

$$
l_{g}(x)=g x \text { e } r_{g}(x)=x g
$$

para cada $x \in G$.

O subespaço das formas diferencias $G$-invariantes à esquerda é um subcomplexo de $\Omega^{\bullet}(G)$ dito de o subcomplexo de formas $G$-invariantes à esquerda, o qual é denotado por $\Omega^{\bullet}(G)_{L}$. De fato,

$$
\Omega^{\bullet}(G)_{L}:=\left\{\omega \in \Omega^{\bullet}(G): l_{g}^{*} \omega=\omega, \forall g \in G\right\}
$$


Note que

$$
l_{g}^{*} d \omega=d\left(l_{g}^{*} \omega\right)=d \omega, \text { se } \omega \in \Omega(G)_{L} .
$$

$\operatorname{Assim}\left(\Omega^{\bullet}(G)_{L}, d^{\bullet}\right)$ é um subcomplexo do usual complexo de De Rham $\left(\Omega^{\bullet}(G), d^{\bullet}\right)$ :

$$
0 \longrightarrow \mathbb{R} \stackrel{\varepsilon}{\longrightarrow} \Omega^{0}(G)_{L} \stackrel{d}{\longrightarrow} \Omega^{1}(G)_{L} \stackrel{d}{\longrightarrow} \Omega^{2}(G)_{L} \longrightarrow \ldots
$$

Analogamente, podemos definir o subcomplexo das formas invariantes a direita denotada por $\left(\Omega^{\bullet}(G)_{R}\right)$. Diremos que uma forma diferencial é dita de invariante se ela é invariante a esquerda e direita e denotamos este subcomplexo por $\Omega(G)_{I}^{\bullet}$.

Notamos que a álgebra de Lie $\mathfrak{g}$ pode-se identificar com o espaço tangente na identidade $e, T_{e} G$, pois cada vetor $v \in T_{e} G$ se estende a um campo vetorial invariante á esquerda definido por $g \longmapsto\left(L_{g}\right)_{e} v$, onde $L_{g}: T G \longrightarrow T G$ denota a derivada da função $l_{g}: G \longrightarrow G$ (resp. $R_{g}: T G \longrightarrow$ $T G$ denota a derivada da funcão $r_{g}: G \longrightarrow G$ ). Dada um $p$-forma diferenciável invariante à esquerda $\omega \in \Omega^{p}(G)_{L}$ e nos podemos só restringirnos ao morfismo $\omega_{e}: \Lambda^{p} T_{e} G \longrightarrow \mathbb{R}$. Isto da um isomorfismo

$$
\begin{aligned}
\varphi: \Omega^{\bullet}(G)_{L} & \longrightarrow \operatorname{Hom}_{\mathbb{R}}\left(\Lambda^{\bullet} \mathfrak{g}, \mathbb{R}\right) \\
\omega & \longmapsto \omega_{e} .
\end{aligned}
$$

De fato, dado um elemento $f \in \operatorname{Hom}_{\mathbb{R}}\left(\Lambda^{p} \mathfrak{g}, \mathbb{R}\right)$ podemos definir uma $p$-forma diferenciável denotada por $\omega^{f} \in \Omega^{p}(G)$ dada por:

$$
\left(\omega^{f}\right)_{g}\left(\left(X_{1}\right)_{g}, \ldots,\left(X_{p}\right)_{g}\right):=f\left(L_{g^{-1}}\left(X_{1}\right)_{g}, \ldots, L_{g^{-1}}\left(X_{p}\right)_{g}\right)
$$

onde $g \in G$ e $X_{1}, \ldots, X_{p}$ são campos vetoriais sobre $G$. A $p$-forma diferenciável $\omega^{f}$ definida acima é invariante à esquerda:

$$
\begin{aligned}
\left(l_{g}^{*} \omega^{f}\right)_{h}\left(\left(X_{1}\right)_{h}, \ldots,\left(X_{p}\right)_{h}\right) & =\left(\omega^{f}\right)_{g h}\left(L_{g}\left(X_{1}\right)_{h}, \ldots, L_{g}\left(X_{p}\right)_{h}\right) \\
& =f\left(L_{(g h)^{-1}} L_{g}\left(X_{1}\right)_{h}, \ldots, L_{(g h)^{-1}} L_{g}\left(X_{p}\right)_{h}\right) \\
& =f\left(L_{h^{-1}}\left(X_{1}\right)_{h}, \ldots, L_{h^{-1}}\left(X_{p}\right)_{h}\right) \\
& =\left(\omega^{f}\right)_{h}\left(\left(X_{1}\right)_{h}, \ldots,\left(X_{p}\right)_{h}\right),
\end{aligned}
$$

e assim $\omega^{f}$ é invariante à esquerda, isto é, $\omega^{f} \in \Omega^{p}(G)_{L}$. Isto prova a sobrejetividade de $\Phi$.

Vejamos a injectividade da $\varphi$. Seja $\omega \in \Omega^{p}(G)_{L}$ tais que $\omega_{e}=0$ e $g \in G$ um elemento quaisquer, nos temos :

$$
\begin{aligned}
\omega_{g}\left(\left(X_{1}\right)_{g}, \ldots,\left(X_{p}\right)_{g}\right) & =\omega_{g}\left(L_{g} L_{g^{-1}}\left(X_{1}\right)_{g}, \ldots, L_{g} L_{g^{-1}}\left(X_{p}\right)_{g}\right) \\
& =\left(l_{g}^{*} \omega\right)_{e}\left(L_{g}^{-1}\left(X_{1}\right)_{g}, \ldots, L_{g^{-1}}\left(X_{p}\right)_{g}\right) \\
& =\omega_{e}\left(L_{g^{-1}}\left(X_{1}\right)_{g}, \ldots, L^{g^{-1}}\left(X_{p}\right)_{g}\right) \\
& =0
\end{aligned}
$$


o qual implica que $\omega=0$, e assim provamos que $\varphi$ é um isomorfismo.

Lembrando a diferencial dada por (2.25). Sejam $\omega$ uma $p$-forma diferenciável invariante à esquerda e $X_{0}, \ldots, X_{p}$ campos vetoriais invariantes à esquerda, então nos temos a formula:

$$
d \omega\left(X_{0}, \ldots, X_{p}\right)=\sum_{0 \leq i<j \leq p}(-1)^{i+j} \omega\left(\left[X_{i}, X_{j}\right], X_{0}, \ldots, \hat{X}_{i}, \ldots, \hat{X}_{j}, \ldots, X_{p}\right) .
$$

Analogamente, seja o complexo $\operatorname{Hom}_{\mathbb{R}}\left(\bigwedge^{*} \mathfrak{g}, \mathbb{R}\right)$ onde $\mathbb{R}$ é o $\mathfrak{g}$-modulo com ação trivial, e assim temos por (2.43):

$$
\mathrm{d} f\left(x_{0} \wedge \ldots \wedge x_{p}\right)=\sum_{0 \leq i<j \leq p} f\left(\left[x_{i}, x_{j}\right] \wedge x_{0} \wedge \ldots \wedge \hat{x}_{i} \wedge \ldots \wedge \hat{x}_{j} \wedge \ldots \wedge x_{p}\right),
$$

onde $f \in \operatorname{Hom}_{\mathbb{R}}\left(\bigwedge^{p} \mathfrak{g}, \mathbb{R}\right)$ e $x_{0}, \ldots x_{p} \in T_{e} G=\mathfrak{g}$. E assim, temos o seguinte diagrama

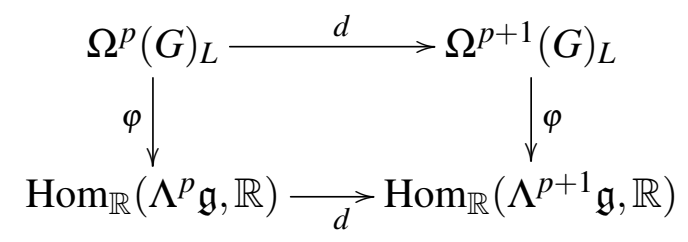

comutativo. O qual da o isomorfismo

$$
H^{*}\left(\Omega^{\bullet}(G)_{L}\right) \cong H^{*}(\mathfrak{g}, \mathbb{R}),
$$

onde $\Omega^{\bullet}(G)_{L}$ denota o complexo das formas diferenciáveis invariantes à esquerda.

Observação 20. Se $G$ é um grupo de Lie compacto conexo, então pelo Teorema 10 nos temos que

$$
H_{D R}^{*}(G, \mathbb{R}) \cong H^{*}\left(\Omega(G)_{L}^{\bullet}\right) \cong H^{*}(\mathfrak{g}, \mathbb{R})
$$

Lema 5. Sejam $G$ um grupo de Lie e u $: G \longrightarrow G$ o mapa inversão da $G$, então

$$
\mathrm{u}^{*} \omega=(-1)^{p} \omega, \quad \forall \omega \in \Omega^{p}(G)_{L} .
$$

Demonstração. Nos temos que

$$
(\mathrm{du})_{g}=-R_{g^{-1}}^{-1} \circ L_{g}^{-1}, \forall g \in G .
$$

Assim, para cada $g \in G$ e $x_{1}, \ldots, x_{p} \in T_{e} G$

$$
\begin{aligned}
\left(\mathrm{u}^{*} \omega\right)_{g}\left(L_{g} x_{1}, \ldots, L_{g} x_{p}\right) & =\omega_{g^{-1}}\left(d \mathrm{u} L_{g} x_{1}, \ldots, d \mathrm{u} L_{g} x_{p}\right) \\
& =\omega_{g^{-1}}\left(-R_{g^{-1}} x_{1}, \ldots,-R_{g^{-1}} x_{p}\right) \\
& =(-1)^{p}\left(R_{g^{-1}}^{*} \omega\right)_{e}\left(x_{1}, \ldots, x_{p}\right) \\
& =(-1)^{p} \omega_{e}\left(x_{1}, \ldots, x_{p}\right) \\
& =(-1)^{p} \omega_{g}\left(L_{g} x_{1}, \ldots, L_{g} x_{p}\right),
\end{aligned}
$$


Assim temos que

$$
\mathrm{u}^{*} \omega=(-1)^{p} \omega
$$

Proposição 17. Seja $G$ um grupo de Lie conexo, então as formas $G$-invariantes são fechados, isto é, $d \omega=0$ para cada $\omega \in \Omega^{p}(G)_{I}$ para cada $p \geq 0$.

Demonstração. Dado que as formas $G$-invariantes formam um subcomplexo do complexo de De Rham, temos

$$
(-1)^{p+1} d \omega=\mathrm{u}^{*} \mathrm{~d} \omega=d \mathrm{u}^{*} \omega=d\left((-1)^{p} \omega\right)=(-1)^{p} \mathrm{~d} \omega, \quad \forall \omega \in \Omega(G)_{I},
$$

então

$$
d \omega=0
$$

Assim nos temos a inclusão $\Omega^{\bullet}(G)_{I} \longrightarrow \Omega^{\bullet}(G)$ e como as formas $G$-invariantes $\Omega^{\bullet}(G)_{I}$ são exatas, então nos temos o seguinte morfismo natural

$$
\Omega^{\bullet}(G)_{I} \longrightarrow H_{D R}^{*}(G) .
$$

Teorema 13. Seja $G$ um grupo de Lie compacto e conexo, então o mapa

$$
\Omega^{\bullet}(G)_{I} \longrightarrow H_{D R}^{*}(G)
$$

é um isomorfismo.

Demonstração. Vide [(GREUB; VANSTONE, 1973), Secção 4.10, Teorema III]

Teorema 14. Sejam $M$ uma variedade diferenciável, $G$ um grupo de Lie compacto e $T: G \times$ $M \longrightarrow M$ uma ação à esquerda de $G$ sobre $M$. Então o homomorfismo induzido pela inclusão

$$
\mathrm{i}_{*}: H^{*}\left(\Omega^{\bullet}(M)^{G}\right) \longrightarrow H_{D R}^{*}(M)
$$

é injetora. Se $G$ é conexo, então $i_{*}$ é um isomorfismo.

Demonstração. Vide [(GREUB; VANSTONE, 1973), Secção 4.3, Teorema I]

\subsubsection{Grupos de Lie semisimples}

Definição 48. Seja $\mathfrak{g}$ uma álgebra de Lie sobre um corpo $K$. Uma forma bilinear simétrica $\kappa: \mathfrak{g} \times \mathfrak{g} \longrightarrow K$ é dita invariante se

$$
\kappa([x, y], z)=\kappa(x,[y, z]),
$$

para cada $x, y, z \in \mathfrak{g}$. 
Definição 49. Seja $\mathfrak{g}$ uma álgebra de Lie sobre um corpo $K$, para cada $x \in \mathfrak{g}$ definimos o morfismo $a d_{x}: \mathfrak{g} \longrightarrow \mathfrak{g}$ o qual é chamado de endomorfismo adjunto dado por:

$$
\operatorname{ad}_{x}(y)=[x, y]
$$

onde $x, y \in \mathfrak{g} \mathrm{e}[$,$] é o colchete da álgebra de Lie \mathfrak{g}$. Ela verifica a seguinte propriedade simples de verificar:

$$
\operatorname{ad}_{[x, y]}=\left[\operatorname{ad}_{x}, \operatorname{ad}_{y}\right], \text { onde } x, y \in \mathfrak{g},
$$

e assim

$$
\begin{aligned}
\operatorname{ad}: \mathfrak{g} & \longrightarrow \operatorname{End}(\mathfrak{g}) \\
x & \longmapsto \operatorname{ad}_{x}
\end{aligned}
$$

é um morfismo de algebras de Lie.

Agora, se g é uma álgebra de Lie de dimensão finita, o traço da composição de dois tais endomorfismos define uma forma bilinear simétrica $\kappa_{\mathfrak{g}}: \mathfrak{g} \times \mathfrak{g} \longrightarrow K$ dada por

$$
\kappa_{\mathfrak{g}}(x, y):=\operatorname{tr}\left(\operatorname{ad}_{x} \circ \operatorname{ad}_{y}\right)
$$

a qual é dita de forma de Cartan-Killing de $\mathfrak{g}$. A forma de Cartan-Killing é invariante:

$$
\kappa_{\mathfrak{g}}([x, y], z)=\kappa_{\mathfrak{g}}(x,[y, z]) .
$$

De fato,

$$
\begin{aligned}
\kappa_{\mathfrak{g}}([x, y], z) & =\operatorname{tr}\left(\operatorname{ad}_{[x, y]} \circ \operatorname{ad}_{z}\right) \\
& =\operatorname{tr}\left(\operatorname{ad}_{x} \circ \operatorname{ad}_{y} \circ \operatorname{ad}_{z}\right)-\operatorname{tr}\left(\operatorname{ad}_{y} \circ \operatorname{ad}_{x} \circ \operatorname{ad}_{z}\right) \\
& =\operatorname{tr}\left(\operatorname{ad}_{x} \circ \operatorname{ad}_{y} \circ \operatorname{ad}_{z}\right)-\operatorname{tr}\left(\operatorname{ad}_{x} \circ \operatorname{ad}_{z} \circ \operatorname{ad}_{z}\right) \\
& =\operatorname{tr}\left(\operatorname{ad}_{x} \circ \operatorname{ad}_{[y, z]}\right) \\
& =\kappa_{\mathfrak{g}}(x,[y, z]) .
\end{aligned}
$$

Proposição 18. Seja $\mathfrak{g}$ uma álgebra de Lie simples, então toda forma bilinear invariante sobre $\mathfrak{g}$ é um múltiplo por um escalar da forma de Cartan-Killing.

Teorema 15 (Critério de semisimplicidade de Cartan). Uma álgebra de Lie g é semisimple se e somente se $\kappa_{\mathfrak{g}}$ é não degenerada.

Demonstração. Vide [(HILGERT; NEEB, 2010), Teorema 5.5.9]

Definição 50. Seja $\mathfrak{g}$ um álgebra de Lie sobre $\mathbb{R}$. Um endomorfismo de álgebra de Lie $\tau: \mathfrak{g} \longrightarrow \mathfrak{g}$ tais que $\tau^{2}=i d$ é dito de involução. Uma involução $\tau: \mathfrak{g} \longrightarrow \mathfrak{g}$ é dita de involução de Cartan sobre $\mathfrak{g}$, se:

$$
\kappa_{\tau}(x, y):=-\kappa_{\mathfrak{g}}(x, \tau(y))
$$

é simétrica e definida positiva, onde $x, y \in \mathfrak{g}$. 
Desde que $\tau$ é uma involução, ela só pode ter autovalores \pm 1 . Denotaremos por $\mathfrak{k}$ ao auto-espaço correspondente ao autovalor 1:

$$
\mathfrak{k}:=\{x \in \mathfrak{g}: \tau(x)=x\},
$$

e denotamos por $\mathfrak{p}$ ao auto-espaço correspondente ao autovalor -1 :

$$
\mathfrak{p}:=\{x \in \mathfrak{g}: \tau(x)=-x\}
$$

E assim temos a descomposição

$$
\mathfrak{g}=\mathfrak{k} \oplus \mathfrak{p}
$$

Observação 21. Se $\tau: \mathfrak{g} \longrightarrow \mathfrak{g}$ é uma involução de Cartan, então se verifica que:

- $\kappa_{\mathfrak{g}}$ é definida negativa sobre $\mathfrak{k}$,

- $\kappa_{\mathfrak{g}}$ é definida positiva sobre $\mathfrak{p}$.

Além disso, temos que $[\mathfrak{k}, \mathfrak{p}] \subset \mathfrak{p}$, isto é, se $x \in \mathfrak{k}$ e $y \in \mathfrak{p}$, então $[x, y] \in \mathfrak{p}$. De fato,

$$
\tau([x, y])=[\tau(x), \tau(y)]=[x,-y]=-[x, y] .
$$

Analogamente, nos temos que $[\mathfrak{k}, \mathfrak{k}] \subset \mathfrak{k},[\mathfrak{p}, \mathfrak{k}] \subset \mathfrak{p},[\mathfrak{p}, \mathfrak{p}] \subset \mathfrak{k}$.

Para cada subgrupo compacto maximal $K$ é associada uma involução de Cartan $\tau: \mathfrak{g} \longrightarrow \mathfrak{g}$ e temos a correspondente descomposição de Cartan de $\mathfrak{g}$ com respeito a $\mathfrak{k}$ :

$$
\mathfrak{g}=\mathfrak{k} \oplus \mathfrak{p},
$$

onde

$$
\mathfrak{k}:=\{x \in \mathfrak{g}: \tau(x)=x\}, \mathfrak{p}:=\{x \in \mathfrak{g}: \tau(x)=-x\} .
$$

a forma bilinear $\kappa_{\mathfrak{g}}$ é definida negativa sobre $\mathfrak{k}$ e definida negativa sobre $\mathfrak{p}$. Além disso,

$$
[\mathfrak{k}, \mathfrak{k}] \subset \mathfrak{k},[\mathfrak{k}, \mathfrak{p}] \subset \mathfrak{p},[\mathfrak{p}, \mathfrak{p}] \subset \mathfrak{k}
$$

Teorema 16 (Descomposição de Cartan). Seja $G$ um grupo de Lie com álgebra de Lie semisimples $\mathfrak{g}, \tau$ uma involução de Cartan de $\mathfrak{g}$, e $\mathfrak{g}=\mathfrak{k} \oplus \mathfrak{p}$ a correspondente descomposição de Cartan. Se $K:=\{g \in G: \tau \circ \operatorname{Ad}(g)=\operatorname{Ad} \circ \tau(g)$, então $L(K)=\mathfrak{k}$, e

$$
\begin{aligned}
\Phi: K \times \mathfrak{p} & \longrightarrow G, \\
(k, x) & \longmapsto k \exp _{G} x
\end{aligned}
$$

é um difeomorfismo. 


\subsubsection{Cohomologia relativa de álgebra de Lie}

Nesta subsecção nos estamos interessado em estudar $X=G(\mathbb{R}) / K$, onde $G$ é um $\mathbb{Q}$-grupo algebraico e $K$ o subgrupo compacto maximal em $G(\mathbb{R})$. Além disso, definir a Cohomologia relativa de álgebra de Lie a qual denotaremos por $H^{*}(\mathfrak{g}, \mathfrak{k} ; V)$ onde $\mathfrak{k}$ é a álgebra de Lie de $U$.

Nos temos que para cada $x \in \mathfrak{g}$, existe um endomorfismo associado $\mathscr{L}_{x}: C^{p}(\mathfrak{g}, V) \longrightarrow$ $C^{p}(\mathfrak{g}, V)$ e um mapa linear $\mathrm{i}_{x}: C^{p}(\mathfrak{g}, V) \longrightarrow C^{p-1}(\mathfrak{g}, V)$ dados por

$$
\begin{gathered}
\left(\mathscr{L}_{x} f\right)\left(x_{1} \wedge \ldots \wedge x_{n}\right)=\sum_{1 \leq i \leq p} f\left(x_{1} \wedge \ldots \wedge\left[x_{i}, x\right] \wedge \ldots \wedge x_{n}\right)+x . f\left(x_{1} \wedge \ldots \wedge x_{n}\right) \\
\left(\mathrm{i}_{x} f\right)\left(x_{1}, \ldots, x_{p-1}\right)=f\left(x \wedge x_{1} \wedge \ldots \wedge x_{p-1}\right)
\end{gathered}
$$

os mapas três mapas estão relacionados pela formula magica de Cartan

$$
\mathscr{L}_{x}=d^{p-1} \circ \mathrm{i}_{x}+\mathrm{i}_{x} \circ d^{p}
$$

Denotamos por $C^{p}(\mathfrak{g}, \mathfrak{k} ; V)$ o subespaço de $C^{p}(\mathfrak{g} ; V)$ dado pelos elementos anulados por $\mathrm{i}_{x}$ e $\mathscr{L}_{x}$, para cada $x \in \mathfrak{k}$ :

$$
C^{p}(\mathfrak{g}, \mathfrak{u} ; V):=\left\{f \in C^{p}(\mathfrak{g}, V): \mathfrak{i}_{x} f=0, \mathscr{L}_{x} f=0, \quad \forall x \in \mathfrak{u}\right\}=\operatorname{Hom}_{\mathfrak{k}}\left(\bigwedge^{p} \mathfrak{g} / \mathfrak{k}, V\right) .
$$

Claramente, se $f \in C^{p}(\mathfrak{g}, \mathfrak{k} ; V)$ implica que $d f \in C^{p+1}(\mathfrak{g}, \mathfrak{k} ; V)$. E assim temos o seguinte complexo

$$
\mathbb{R} \longrightarrow C^{\bullet}(\mathfrak{g}, \mathfrak{k} ; V): 0 \longrightarrow \mathbb{R} \stackrel{d}{\longrightarrow} C^{1}(\mathfrak{g}, \mathfrak{k} ; V) \stackrel{d}{\longrightarrow} C^{2}(\mathfrak{g}, \mathfrak{k} ; V) \longrightarrow \ldots
$$

e assim definimos $H^{p}(\mathfrak{g}, \mathfrak{k} ; V):=H^{p}\left(C^{*}(\mathfrak{g}, \mathfrak{k} ; V)\right)$.

Seja $G$ um grupo de Lie conexo, $K$ um subgrupo compacto maximal de $G$. Seja $X:=G / K$ o espaço homogêneo. Então pelo Teorema 16 temos que $X$ é difeomorfo a um espaço vetorial. $\mathrm{E}$ assim contráctil. Existe uma ação à direita de $G$ sobre $X$ definida por

$$
\begin{aligned}
& \mathrm{r}: X \times G \longrightarrow X \\
& (U x, g) \longmapsto U x g .
\end{aligned}
$$

Esta ação induz uma ação à esquerda de $G$ sobre o complexo de De Rham $\Omega^{*}(X, \mathbb{R})$

Observação 22. O complexo

$$
0 \longrightarrow \mathbb{R} \longrightarrow \Omega^{0}(X, \mathbb{R}) \longrightarrow \Omega^{1}(X, \mathbb{R}) \longrightarrow \ldots
$$

é uma resolução injectiva de $G$-módulos para $\mathbb{R}$. Por tanto o grupo de cohomologia contínua do $G$-modulo $\mathbb{R}$ é o grupo de cohomologia do complexo $\Omega^{\bullet}(X, \mathbb{R})^{G}$ (como na definição de G-invariantes). Isto é

$$
H_{\text {cont }}^{*}(G, \mathbb{R}) \cong H^{*}\left(\Omega^{\bullet}(X, \mathbb{R})^{G}\right)
$$


A proposição seguinte da uma interpretação geométrica da Cohomologia relativa das álgebras de Lie:

Proposição 19. Seja $G$ grupo de Lie conexo, $K$ subgrupo compacto maximal de $G$ e $X=G / K$. Seja $\mathfrak{g}$ e $\mathfrak{k}$ as álgebras de Lie de $G$ e $K$. O isomorfismo $\varphi: \Omega^{\bullet}(G, \mathbb{R})_{L} \longrightarrow C^{\bullet}(\mathfrak{g}, \mathbb{R})$ e a aplicação

$$
\begin{aligned}
\pi^{*}: \Omega^{\bullet}(X) & \longrightarrow \Omega^{\bullet}(G) \\
\omega & \longmapsto \pi^{*} \omega,
\end{aligned}
$$

onde $\pi: G \longrightarrow G / K$ é a projeção. Elas induzem um isomorfismo natural

$$
\Omega^{\bullet}(X, \mathbb{R})^{G} \longrightarrow C^{\bullet}(\mathfrak{g}, \mathfrak{k}, \mathbb{R})
$$

Por tanto, nos temos o seguinte isomorfismo de cohomologia

$$
H^{*}\left(\Omega^{\bullet}(X)^{G}\right) \cong H^{*}(\mathfrak{g}, \mathfrak{k} ; \mathbb{R})
$$

Demonstração. Segue imediato da definição da $\varphi$.

Observação 23. Se nas hipótese da Proposição 19 adicionamos a condição de que $G$ seja compacto, então pelo Teorema 14 temos o isomorfismo

$$
H_{D R}^{*}(X, \mathbb{R}) \cong H^{*}(\mathfrak{g}, \mathfrak{u} ; \mathbb{R})
$$

Teorema 17 (Van Est). Seja $G$ um grupo de Lie conexo, $U$ um subgrupo compacto maximal. Sejam $g$ e $u$ as álgebras de Lie de $G$ e $U$, existe um isomorfismo

$$
\beta: H_{\text {cont }}^{*}(G, \mathbb{R}) \longrightarrow H^{*}(\mathfrak{g}, \mathfrak{u}, \mathbb{R})
$$

Demonstração. Segue imediato da observação 22 e a proposição 19 .

Definição 51. Seja $\mathfrak{g}$ uma álgebra de Lie e seja $j: \mathfrak{h} \longrightarrow \mathfrak{g}$ uma subálgebra. Dizemos que $\mathfrak{h}$ é reduzido em $\mathfrak{g}$ se a representação $\theta_{\mathfrak{h}}$ é semisimples. Dizemos que $\mathfrak{h}$ é noncohomologous to zero se é reduzido em $\mathfrak{g}$ e o morfismo

$$
j^{*}: H^{*}(\mathfrak{g}) \longrightarrow H^{*}(\mathfrak{h})
$$

é sobrejetivo

Proposição 20. Seja h uma noncohomologous to zero subálgebra de $\mathfrak{g}$. Então o morfismo

$$
H(\mathfrak{g}, \mathfrak{h})^{*} \longrightarrow H^{*}(\mathfrak{g})
$$

é injectivo. 


\subsection{Cálculo da Cohomologia contínua}

Nesta secção apresentaremos o isomorfismo de Van Est o qual permite calcular a cohomologia contínua de um grupo de Lie.

Seja $G$ um grupo algébrico redutivo definido sobre $\mathbb{R}$. Nos assumimos que $G(\mathbb{R})$ é um grupo de Lie conexo. Então $G(\mathbb{C})$ é um grupo redutivo conexo complexo. Seja $\mathfrak{g}_{\mathbb{R}}$ a álgebra de Lie de $G(\mathbb{R})$. Então $\mathfrak{g}_{\mathbb{C}}=\mathfrak{g}_{\mathbb{R}} \otimes \mathbb{C}$ é a álgebra de Lie de $G(\mathbb{C})$.

Seja $K$ um subgrupo compacto maximal de $G(\mathbb{R})$, seja k a álgebra de Lie de $K$ e seja

$$
\mathfrak{g}_{\mathbb{R}}=\mathfrak{k} \oplus \mathfrak{p}
$$

a descomposição de Cartan de $\mathfrak{g}_{\mathbb{R}}$ com respeito a k. Então

$$
\mathfrak{g}_{u}=\mathfrak{k} \oplus \mathfrak{i p} \subset \mathfrak{g}_{\mathbb{C}}
$$

é uma forma compacta de $\mathfrak{g}_{\mathbb{R}}$ (Ver (Springer Monographs in Mathematics) Joachim Hilgert, Karl-Hermann Neeb (auth.)-Structure and Geometry of Lie Groups -Springer-Verlag New York (2012), Proposition 13.2.5.). Por tanto, o grupo de Lie correspondente $G_{u}$ é um subgrupo compacto maximal de $G(\mathbb{C})$ que contem a $K$.

Denotaremos por $X:=G(\mathbb{R}) / K$ e $X_{u}:=G_{u} / K, X_{u}$ é dita de compacto gêmeo do espaço simétrico $X$ o qual é determinado pelo par $(G(\mathbb{R}), K)$. Desde que $X_{u}$ é determinado a menos de isomorfismos pelo grupo $G(\mathbb{R})$, e todas as aplicações são fixadas pelo subgrupo compacto $K$, nos denotamos $X_{u}$ por $C T(G(\mathbb{R}))$ e dito de compacto gêmeo de $G(\mathbb{R})$. Agora consideramos as ações de $G(\mathbb{R})$ sobre $X$ e $G_{u}$ sobre $X_{u}$ dadas por $(g, p) \mapsto p g$. Segue da Proposição 19 , o complexo $C^{*}\left(\mathfrak{g}_{\mathbb{R}}, \mathfrak{k}, \mathbb{R}\right)$ é isomorfo ao complexo das formas $G(\mathbb{R})$-invariantes sobre $X$ e $C^{*}\left(\mathfrak{g}_{u}, \mathfrak{k}, \mathbb{R}\right)$ ao complexo das formas $G_{u}$-invariantes sobre $C T(G(\mathbb{R}))$. Desde que $G_{u}$ é o subgrupo compacto maximal, pela Observação 23 temos o isomorfismo

$$
\alpha: H_{D R}^{*}(C T(G(\mathbb{R})), \mathbb{R}) \longrightarrow H^{*}\left(\mathfrak{g}_{u}, \mathfrak{k}, \mathbb{R}\right) .
$$

Seja

$$
l: C^{*}\left(\mathfrak{g}_{u}, \mathfrak{k} ; \mathbb{R}\right) \longrightarrow C^{*}(\mathfrak{g}, \mathfrak{k} ; \mathbb{R})
$$

um isomorfismo que envia $f \in C^{p}\left(\mathfrak{g}_{u}, \mathfrak{k} ; \mathbb{R}\right)=\operatorname{Hom}_{\mathfrak{k}}\left(\Lambda^{p} i \mathfrak{p}, \mathbb{R}\right)$ a $l(f)=i^{p} f \in C^{p}(\mathfrak{g}, \mathfrak{k}, \mathbb{R})=$ $\operatorname{Hom}_{\mathfrak{k}}\left(\Lambda^{p} \mathfrak{p}, \mathbb{R}\right)$,

$$
l(f)\left(x_{1} \wedge \ldots \wedge x_{p}\right):=f\left(i x_{1} \wedge \ldots \wedge i x_{p}\right)
$$

onde $x_{1}, \ldots, x_{p} \in \mathfrak{p}$. Denotaremos por $\gamma^{\prime}$ a composição

$$
\gamma^{\prime}: H_{D R}^{*}(C T(G(\mathbb{R})), \mathbb{R}) \stackrel{\alpha}{\longrightarrow} H^{*}\left(\mathfrak{g}_{u}, \mathfrak{k}, \mathbb{R}\right) \stackrel{l^{*}}{\longrightarrow} H^{*}\left(\mathfrak{g}_{\mathbb{R}}, \mathfrak{k}, \mathbb{R}\right) \stackrel{\beta^{-1}}{\longrightarrow} H_{\text {cont }}^{*}(G(\mathbb{R}), \mathbb{R}),
$$

onde $\beta^{-1}$ é a inversa do isomorfismo de Van Est.

Desde que $\alpha, \beta^{-1}$ e $j$ são isomorfismos, temos que $\gamma^{\prime}$ é um isomorfismo. Por tanto, nos podemos usar o fato que a cohomologia de grupos de Lie clássicos e seus espaços homogêneos associados são conhecidas, para calcular a cohomologia contínua de grupo dos grupos de Lie clássicos. 



\section{K-TEORIA ALGÉBRICA DE ANÉIS}

Neste capitulo definiremos os $K$-grupos clássicos $K_{0}, K_{1}$ e daremos a definição geral dos $K$-grupos de um anel dada por Quillen.

\subsection{Grupo $K_{0}$ de um anel}

Lembrando que um monoide abeliano é um conjunto $M$ munido de uma operação $(+)$ associativa, comutativa e um elemento identidade 0 . Um morfismo de monoides $f: M \longrightarrow N$ é um morfismo de conjuntos tais que $f(0)=0$ e $f\left(m+m^{\prime}\right)=f(m)+f\left(m^{\prime}\right)$.

Exemplo 10. O exemplo mais conhecido de monoide um abeliano é $\mathbb{N}=\{0,1,2, \cdots\}$, os números naturais com elemento identidade 0 .

Definição 52. O grupo realizado de um monoide abeliano $M$ é um grupo abeliano $M^{-1} M$ munido de um morfismo de monoides $f: M \longrightarrow M^{-1} M$ que verifica a seguinte propriedade universal, para todo grupo abeliano $A$ e todo morfismo de monoides $\alpha: M \longrightarrow A$, existe um único morfismo de grupos abelianos $\bar{\alpha}: M^{-1} M$ tas que o seguinte diagrama

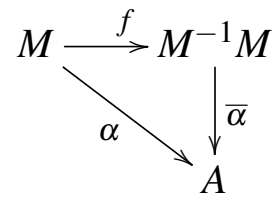

é comutativo.

Todo monoide abeliano $M$ tem um grupo realizado. Uma maneira de construir é tomar o quociente do grupo abeliano livre $F(M)$ com símbolos $[m], m \in M$ pelo subgrupo $R(M)$ gerado pelas relações $[m+n]-[m]-[n]$. Agora, dado um morfismo de monoides $M \longrightarrow N$, o mapa $M \longrightarrow N \longrightarrow N^{-1} N$ se estende unicamente para um morfismo $M^{-1} M \longrightarrow N^{-1} N$. Assim o mapa $M \longmapsto M^{-1} M$ é um functor da categoria de monoides abelianos à categoria de grupos abelianos. 
Também pode-se ver que é adjunto a esquerda do functor esquecedor, devido ao isomorfismo natural

$$
\operatorname{Hom}_{\text {monoide abeliano }}(M, A) \cong \operatorname{Hom}_{\mathrm{Ab}}\left(M^{-1} M, A\right) \text {. }
$$

Proposição 21. Seja $M$ um monoide abeliano. Então:

(1) Todo elemento de $M^{-1} M$ é da forma $[m]-[n]$ para algum $m, n \in M$,

(2) Se $m, n \in M$, então $[m]=[n]$, se e somente se $m+p=n+p$ para algum $p \in M$,

(3) O morfismo de monoides $M \times M \longrightarrow M^{-1} M$ mandando $(m, n)$ a $[m]-[n]$ é sobrejetivo.

(4) Por tanto $M^{-1} M$ é o quociente (como conjunto) de $M \times M$ pela relação de equivalência gerada por $(m, n) \sim(m+p, n+p)$.

Demonstração. Todo elemento de um grupo abeliano livre é uma diferencia de suma de seus gerados, e em $F(M)$ nos temos $\left[m_{1}\right]+\left[m_{2}\right]+\cdots \equiv\left[m_{1}+m_{2}+\cdots\right]$ modulo $R(M)$. Portanto todo elemento de $M^{-1} M$ é uma diferencia de geradores. Esto prova (1) e (3). Para (2), suponha que $[m]-[n]=0$ em $M^{-1} M$. Então no grupo abeliano livre $F(M)$ nos temos

$$
[m]-[n]=\sum\left(\left[a_{i}+b_{i}\right]-\left[a_{i}\right]-\left[b_{i}\right]\right)-\sum\left(\left[c_{j}+d_{j}\right]-\left[c_{j}\right]-\left[d_{j}\right]\right),
$$

e assim temos

$$
[m]+\sum\left(\left[a_{i}\right]+\left[b_{i}\right]\right)+\sum\left[c_{j}+d_{j}\right]=[n]+\sum\left[a_{i}+b_{i}\right]+\sum\left(\left[c_{j}\right]+\left[d_{j}\right]\right) .
$$

Agora em um grupo abeliano livre, duas sumas de geradores $\sum\left[x_{i}\right]$ e $\sum\left[y_{j}\right]$ sao iguais se, elas tem o mesmo numero de termos e existe uma permutação $\sigma$ tais que $y_{i}=x_{\sigma(i)}$. Por tanto os gerado do lado esquerdo e direito de 3.2 sua iguais salvo uma permutação. Isto podemos entender que em $M$ a suma dos termos do lado esquerdo e direito de 3.2 são os mesmos, isto é ,

$$
m+\sum\left(a_{i}+b_{i}\right)+\sum\left(c_{j}+d_{j}\right)=n+\sum\left(a_{i}+b_{i}\right)+\sum\left(c_{j}+d_{j}\right)
$$

em $M$. O qual conclui (2), a parte (4) segue de (1) e (2).

Definição 53. Seja $R$ um anel.E seja $P(R)$ o conjunto das classes de isomorfismo dos $R$-módulos projetivos finitamente gerados munido da suma direta $\oplus$ e o elemento identidade 0 , o qual é um monoide abeliano. O grupo de Grothendieck de $R$, denotado por $K_{0}(R)$, é o grupo realizado de $P(R)$.

Observação 24. Se $R$ é uma anel comutativo, $K_{0}(R)$ é um anel comutativo com $1=[R]$, porque o monoide $P(R)$ é um comutativo semianel com produto $\otimes_{R}$. Isto segue do seguinte fato: $\otimes_{R}$ é distributivo sobre $\oplus ; P \otimes_{R} L \cong L \otimes_{R} P$ e $P \otimes_{R} R \cong P$; se $P, L$ são $R$-módulos finitamente gerados, então $P \otimes_{R} L$ também é um R-modulo projetivo finitamente gerado. 
Exemplo 11. Seja $K$ um corpo. Então o monoide abeliano $P(K)$ é isomorfo a $\mathbb{N}$, assim $K_{0}(K)=$ $\mathbb{Z}$. Um argumento similar é usado para mostrar que $K_{0}(R)=\mathbb{Z}$ para todo anel local $R$ e também para todo Dominio de ideais principais (DIP). Em particular $K_{0}(\mathbb{Z})=\mathbb{Z}$.

$K_{0}$ é um functor da categoria de anéis à categoria de grupos topológicos e da categoria de anéis comutativos à categoria de anéis comutativos. Para ver isto, suponhamos que $R \longrightarrow S$ é um morfismo de anéis, o mapa $\otimes_{R} S: P(R) \longrightarrow P(S)$ definido por $P \longmapsto P \otimes_{R} S$ é um morfismo de monoides, e por tanto um morfismo de grupo $\otimes_{R} S: K_{0}(R) \longrightarrow K_{0}(S)$. Se $R, S$ são anéis comutativos, então $\otimes_{R} S: K_{0}(R) \longrightarrow K_{0}(S)$ é um morfismo de anéis, pois $\otimes_{R} S: P(R) \longrightarrow P(S)$ é um morfismo de semianéis:

$$
\left(P \otimes_{R} L\right) \otimes_{R} S \cong\left(P \otimes_{R} S\right) \otimes_{S}\left(L \otimes_{R} S\right)
$$

\subsection{Grupo $K_{1}$ de um anel}

Seja $R$ um anel associativo com unidade . Consideremos o grupo $G L_{n}(R)$ das $n \times n$ matrizes invertíveis com coeficientes em $R$. Denotamos $e_{i j}^{(n)}(\lambda)$ para cada $\lambda \in R$ e $1 \leq i, j \leq n$, $i \neq j$ a matriz $n \times n$ tendo 1 's na diagonal e 0 's fora, exceto na posição $(i, j)$ onde tem a $\lambda$. Tais matrizes são ditas de elementares

$$
i \quad\left(\begin{array}{ccccccc}
1 & \cdots & 0 & \cdots & 0 & \cdots & 0 \\
\vdots & \ddots & \vdots & \vdots & \vdots & \vdots & \vdots \\
0 & \cdots & 1 & \cdots & 0 & \cdots & 0 \\
\vdots & \vdots & \vdots & \ddots & \vdots & \vdots & \vdots \\
0 & 0 & \lambda & \cdots & 1 & \cdots & 0 \\
\vdots & \vdots & \vdots & \vdots & \vdots & \ddots & \vdots \\
0 & 0 & 0 & \cdots & 0 & \cdots & 1
\end{array}\right)
$$

Existem as seguintes relações para matrizes elementais.

1. $e_{i j}^{(n)}(\lambda) e_{i j}^{(n)}(\mu)=e_{i j}^{(n)}(\lambda+\mu), \forall \lambda, \mu \in R$.

2. $\left[e_{i j}^{(n)}(\lambda), e_{j k}^{(n)}(\mu)\right]=e_{i k}^{(n)}(\lambda \mu)$, para $i \neq k$.

3. $\left[e_{i j}^{(n)}(\lambda), e_{k l}^{(n)}(\mu)\right]=1$, para $j \neq k, i \neq l$.

Tais matrizes geram o subgrupo de matrizes elementais $E_{n}(R) \subseteq G L_{n}(R)$. Temos o morfismo natural definido por

$$
\begin{aligned}
\varphi: G L_{n}(R) & \hookrightarrow G L_{n+1}(R) \\
M & \mapsto\left(\begin{array}{cc}
M & 0 \\
0 & 1
\end{array}\right) .
\end{aligned}
$$


o qual induz o morfismo

$$
\varphi: E_{n}(R) \hookrightarrow E_{n+1}(R) .
$$

Por tanto, nos podemos definir os limites direitos

$$
\begin{aligned}
G L(R) & :=\lim _{\longrightarrow} G L_{n}(R) \\
E(R) & :=\stackrel{\lim }{\longrightarrow} E_{n}(R) .
\end{aligned}
$$

O grupo $G L(R)$ pode ser entendido como o grupo de matrizes arbitrariamente grande. Para a multiplicação de matrizes de diferentes tamanhos, usamos o morfismo $\varphi$.

Observação 25. Das relações anteriores temos que $\left[E_{n}(R), E_{n}(R)\right]=E_{n}(R)$ para $n \geq 3$. E assim temos que $E(R)=[E(R), E(R)]$ o qual diz que $E(R)$ é perfeito.

Lema 6 (Whitehead). Para cada matriz $A \in G L_{n}(R)$ temos que

$$
\left(\begin{array}{cc}
A & 0 \\
0 & A^{-1}
\end{array}\right) \in E_{2 n}(R)
$$

Demonstração. Para cada $B \in M_{n}(R)$, claramente $\left(\begin{array}{cc}I_{n} & B \\ 0 & I_{n}\end{array}\right),\left(\begin{array}{cc}I_{n} & 0 \\ B & I_{n}\end{array}\right) \in E_{2 n}(R)$. E usando a identidade

$$
\left(\begin{array}{cc}
A & 0 \\
0 & A^{-1}
\end{array}\right)=\left(\begin{array}{cc}
I_{n} & 0 \\
A^{-1}-I_{n} & I_{n}
\end{array}\right)\left(\begin{array}{cc}
I_{n} & I_{n} \\
0 & I_{n}
\end{array}\right)\left(\begin{array}{cc}
I_{n} & 0 \\
A-I_{n} & I_{n}
\end{array}\right)\left(\begin{array}{cc}
I_{n} & -A^{-1} \\
0 & I_{n}
\end{array}\right)
$$

Proposição 22. Seja $R$ um anel, então

$$
E(R)=[E(R), E(R)]=[G L(R), G L(R)]
$$

Demonstração. Sejam $M, N \in G L(R)$ sem perda de generalidade, nós podemos supor que $M, N \in$ $G L_{n}(R)$

$$
\left(\begin{array}{cc}
{[M, N]} & 0 \\
0 & 1
\end{array}\right)=\left(\begin{array}{cc}
M N M^{-1} N^{-1} & 0 \\
0 & 1
\end{array}\right)=\left(\begin{array}{cc}
M N & 0 \\
0 & (M N)^{-1}
\end{array}\right)\left(\begin{array}{cc}
M^{-1} & 0 \\
0 & M
\end{array}\right)\left(\begin{array}{cc}
N^{-1} & 0 \\
0 & N
\end{array}\right)
$$

e segue do Lema de Whitehead que $[M, N] \in E(R)$ o qual implica que $[G L(R), G L(R)] \subseteq E(R)$. Portanto temos que $[G L(R), G L(R)]=E(R)$, pois

$$
E(R)=[E(R), E(R)] \subseteq[G L(R), G L(R)]
$$


A não comutatividade de $G L(R)$ é medida por $E(R)=[G L(R), G L(R)]$. Esto sugere, que se deve estudar o abelianizador de $G L(R)$.

Definição 54. Seja R um anel, o grupo $K_{1}$ é dado por

$$
K_{1}(R):=G L(R) / E(R)=G L(R)^{\mathrm{ab}}=H_{1}(G L(R),(Z)) .
$$

Pelo lema de Whitehead, o produto $[M][N]:=[M N]$ em $K_{1}(R)$. Pode ser visto como a "matriz escalonada"

$$
\left(\begin{array}{cc}
M N & 0 \\
0 & 1
\end{array}\right)=\left(\begin{array}{cc}
M & 0 \\
0 & N
\end{array}\right)\left(\begin{array}{cc}
N & 0 \\
0 & N^{-1}
\end{array}\right)
$$

então $[M N]=\left(\begin{array}{cc}M & 0 \\ 0 & N\end{array}\right)$ em $K_{1}(R)$.

Observação 26. O homomorfismo de grupos det : $G L_{n}(R) \longrightarrow R^{\times}$pode ser estendido a det : $G L(R) \longrightarrow R^{\times}$o qual é um homomorfismo de grupos, pois se $M, N \in G L(R)$ temos que

$$
\operatorname{det}\left(\begin{array}{cc}
M & 0 \\
0 & 1
\end{array}\right)=\operatorname{det}(M) .
$$

Para mais detalhes veja-se (ARTIN, 1957).

Definição 55. Seja $R$ um anel, o grupo linear especial $S L(R)$ é definido por

$$
S L(R):=\{M \in G L(R): \operatorname{det}(M)=1\} .
$$

Vemos que $E(R) \subseteq S L(R)$, então $[E(R), E(R)] \subseteq[S L(R), S L(R)] \subseteq[G L(R), G L(R)]$, segue da Proposição 22 que $E(R)=[S L(R), S L(R)]$.

Seja $S K_{1}(R):=S L(R) /[S L(R), S L(R)]=S L(R) / E(R)$. Nos temos uma sequencia exata curta que cinde

$$
0 \longrightarrow S L(R) \hookrightarrow G L(R) \stackrel{\text { det }}{\longrightarrow} R^{\times} \longrightarrow 0
$$

O qual implica que $G L(R) \cong S L(R) \oplus R^{\times}$(a secção é dada pela inclusão $R^{\times}=G L_{1}(R) \hookrightarrow G L(R)$ ), e como $E(R) \subseteq S L(R)$, então a sequencia (3.11) induz a sequencia exata curta que cinde

$$
0 \longrightarrow S K_{1}(R) \longrightarrow K_{1}(R) \stackrel{\operatorname{det}}{\longrightarrow} R^{\times} \longrightarrow 0,
$$

isto é, $K_{1}(R) \cong S K_{1}(R) \oplus R^{\times}$. Neste trabalho, nos estamos interessados no caso quando $R=\mathscr{O}_{F}$, onde $\mathscr{O}_{F}$ o anel de inteiros de um corpo numérico $F$.

Teorema 18 (Bass-Milnor-Serre). Seja $F$ um corpo numérico e seja $\mathscr{O}_{F}$ seu anel de inteiros. Então $S K_{1}\left(\mathscr{O}_{F}\right)=0$. Em particular, $K_{1}\left(\mathscr{O}_{F}\right) \cong \mathscr{O}_{F}^{\times}$. 


\subsection{O teorema da unidade}

\subsubsection{Integralidade}

Um corpo numérico é uma extensão $F$ de $\mathbb{Q}$. Um elemento $y \in F$ é dito integral, se $y$ é zero de um polinômio mônico $f(X) \in \mathbb{Z}[X]$. Daremos a definição de integralidade de um fato mais geral.

Definição 56. Seja $A \subseteq B$ uma extensão de anéis. Um elemento de $b \in B$ é dito integral sobre $A$, se satisfaz uma equação mônica

$$
x^{n}+a_{n-1} x^{n-1}+\cdots+a_{0}=0, n \geq 1,
$$

com coeficientes $a_{i} \in A$. O anel $B$ é dito integral sobre $A$ se todos os elementos $b \in B$ são integrais sobre $A$.

Proposição 23. Seja $A \subseteq B$ uma extensão de anéis. Um numero finito de elementos $b_{1}, b_{2}, \cdots, b_{r} \in$ $B$ são integrais sobre $A$ se e somente se $A\left[b_{1}, b_{2}, \cdots, b_{r}\right]$ visto como $A$-módulo é finitamente gerado.

Demonstração. Vide [(NEUKIRCH, 1937), Proposição 2.2]

Observação 27. Seja $A \subseteq B$ uma extensão de anéis. Dados $x, y \in B$ integrais sobre $A$, então pela Proposição 23 temos que $A[x, y]$ é um $A$-módulo finitamente gerado. Além disso, vemos que $A[x, y, x y]=A[x, y, x+y]=A[x, y]$ e pela Proposição 23 temos que $x+y, x y \in B$ são integrais sobre $A$.

Proposição 24. Seja $A \subseteq B \subseteq C$ duas extensões de anéis. Se $C$ é integral sobre $B$ e $B$ é integral sobre $A$, então $C$ é integral sobre $A$.

Demonstração. Seja $c \in C$, então existe uma equação mônica

$$
c^{n}+b_{n-1} c^{n-1}+\cdots+b_{1} c+b_{0}=0
$$

$\operatorname{com} b_{i} \in B$ e como $B$ é integral sobre $A$, então pela Proposição 23 temos que $A\left[b_{1}, \cdots, b_{n-1}\right]$ é um $A$-modulo finitamente gerado. E por tanto temos que $c \in C$ é integral sobre $A\left[b_{1}, \cdots, b_{n-1}\right]$, novamente pela Proposição 23 temos que $A\left[b_{1}, \cdots, b_{n-1}, c\right]$ é um $A\left[b_{1}, \cdots, b_{n-1}\right]$-módulo finitamente gerado e como $A\left[b_{1}, \cdots, b_{n-1}\right]$ é um $A$-módulo finitamente gerado, então $A\left[b_{1}, \cdots, b_{n-1}, c\right]$ é um $A$-módulo finitamente gerado e assim $c$ é integral sobre $A$. Por tanto $C$ é integral sobre $A$.

Dada $A \subseteq B$ uma extensão de anéis, pela Observação 27 temos provado que o conjunto dos elementos integrais de $B$ sobre $A$

$$
\bar{A}:=\{b \in B: b \text { é integral sobre } A\}
$$


formam um anel o qual é dito de fecho integral de A sobre B. Dizemos que $A$ é integralmente fechado sobre $B$, se $A=\bar{A}$. Segue da Proposição 23 que $\bar{A}$ é integralmente fechado sobre $B$. Se $A$ é um domínio integral com corpo de frações $K$, então o fecho integral $\bar{A}$ de $A$ sobre $K$ é dita de normalização de $A$, e $A$ é dito simplesmente integralmente fechado se $A=\bar{A}$.

Observação 28. Todo Domínio de fatoração única $D$ é integralmente fechado. De fato, sejam $K$ o corpo de fracões de $D$ e $a / b \in K(a, b \in D)$ é integral sobre $D$, isto é

$$
(a / b)^{n}+a_{n-1}(a / b)^{n-1}+\cdots+a_{0}=0,
$$

$\operatorname{com} a_{i} \in D$, então

$$
a^{n}+a_{n-1} a^{n-1} b+\cdots+a_{0} b^{n}=0
$$

assim todo elemento primo $p$ que divide a $b$ também divide a $a$, tomando $a / b$ reduzido temos que $a / b \in D$. Por tanto $D$ é integralmente fechado.

Lema 7. Seja $A$ um domínio integralmente fechado, $K$ seu corpo de fracões, $L \mid K$ uma extensão finita de corpos , $B$ o fecho integral de $A$ em $L$. Então $B$ é integralmente fechado.

Demonstração. Primeiro vejamos que cada elemento de $L$ é da forma $b / a \operatorname{com} b \in B$ e $a \in A$. Seja $l \in L$ como $L \mid K$ é uma extensão finita, então temos que

$$
l^{n}+k_{n-1} l^{n-1}+\cdots+k_{0}=0
$$

com $k_{i} \in K$ e como $K$ é o corpo de fracões de $A$, temos que

$$
a_{n} l^{n}+a_{n-1} l^{n-1}+\cdots+a_{0}=0,
$$

com $a_{i} \in A$, multiplicando por $a^{n-1}$ temos que $a_{n} l \in L$ é integral sobre $A$. E assim $b=a_{n} l \in B$, isto é $l=b / a_{n} \operatorname{com} b \in B$ e $a_{n} \in A$. Isto mostra que $L$ é o corpo de frações de $B$ e como $B$ é o fecho integral de $A$ em $L$, então $B=\bar{B}$. Por tanto, $B$ é integralmente fechado.

Lema 8. Sejam $A, B$ e $L$ como acima. Então, $\beta \in L$ é integral sobre $A$ se e somente se o polinômio minimal $p(X) \in K[X]$ tem seus coeficientes em $A$.

Demonstração. Seja $\beta \in L$ zero do polinômio $g(X) \in A[X]$. Então $p(X)$ divide a $g(X)$ em $K[X]$, todas as raízes $\beta_{1}, \beta_{2}, \cdots, \beta_{n}$ são integrais sobre $A$, e assim todos os coeficientes de $p(X) \in K[X]$ são integrais sobre $A$. Por tanto $p(X) \in A[X]$.

Definição 57. Seja $L \mid K$ uma extensão finita de corpos. O traço e a norma de um elemento $x \in L$ são dadas pelo traço e a determinante, respectivamente, do endomorfismo

$$
\begin{aligned}
T_{x}: L & \longrightarrow L \\
\alpha & \longmapsto x \alpha,
\end{aligned}
$$

do $K$-espaço vetorial $L$. Isto é, $\operatorname{Tr}_{L \mid K}(x):=\operatorname{Tr}\left(T_{x}\right)$ e $N_{L \mid K}(x):=\operatorname{det}\left(T_{x}\right)$. 
No polinômio característico de $T_{x}$, é dado por

$$
f_{x}(t)=\operatorname{det}\left(t I_{n}-T_{x}\right)=t^{n}-k_{1} t^{n-1}+\cdots+(-1)^{n} k_{n} \in K[t],
$$

onde $n=[L: K]$, reconhecemos o traço e a norma por $a_{1}=\operatorname{Tr}_{L \mid K}(x)$ e $a_{n}=N_{L \mid K}(x)$. Desde que $T_{x+y}=T_{x}+T_{y}$ e $T_{x y}=T_{x} \circ T_{y}$, obtemos os homomorfismo

$$
\operatorname{Tr}_{L \mid K}: L \longrightarrow K \text { e } N_{L \mid K}: L^{\times} \longrightarrow K^{\times}
$$

No caso onde a extensão $L \mid K$ é separável, a traça e a norma tem uma interpretação mediante a Teoria de Galois.

Proposição 25. Se $L \mid K$ é uma extensão finita e separável e $\sigma: L \longrightarrow \bar{K}$ varia sobre os $K$ mergulhos de $L$ na clausura algebraica $\bar{K}$ de $K$, então nos temos

(i) $f_{x}(t)=\prod_{\sigma}(t-\sigma x)$,

(ii) $\operatorname{Tr}_{L \mid K}(x)=\sum_{\sigma} \sigma(x)$,

(iii) $N_{L \mid K}(x)=\prod_{\sigma} \sigma(x)$.

Demonstração. Vide [(NEUKIRCH, 1937), Proposição 2.6]

Definição 58. Um sistema de elementos $\omega_{1}, \cdots, \omega_{n} \in B$ tal que, para cada $b \in B$ pode ser escrito de maneira única como combinação linear

$$
b=a_{1} \omega_{1}+\cdots+a_{n} \omega_{n}
$$

com coeficientes em $a_{i} \in A$, é dita de base integral de $B$ sobre $A$ (ou uma $A$-base de $B$ ). Uma base integral de $B$ sobre $A$ é também uma base de $L$ sobre $K$, a cardinalidade $n$ sempre é igual ao grau $[L: K]$ da extensão.

Proposição 26. Sejam $L \mid K$ uma extensão finita e separável, $A$ um DIP, então todo $B$-módulo $M \neq 0$ de $L$ é um $A$-módulo livre de posto $[L: K]$. Em particular, $B$ admite uma base integral sobre $A$.

Demonstração. Vide [(NEUKIRCH, 1937), Proposição 2.10]

Observação 29. Nosso principal interesse nas considerações de integralidade é estudar o fecho integral $\mathscr{O}_{F} \subseteq F$ de $\mathbb{Z} \subseteq \mathbb{Q}$ em um corpo numérico $F$. Pela Proposição 26, todo $\mathscr{O}_{F}$-módulo finitamente gerado a de $F$ admite uma $\mathbb{Z}$ base $\alpha_{1}, \cdots, \alpha_{n}$,

$$
\mathfrak{a}=\mathbb{Z} \alpha_{1}+\cdots+\mathbb{Z} \alpha_{n}
$$

Teorema 19. Seja $F$ um corpo numérico. $\mathrm{O}$ anel $\mathscr{O}_{F}$ é noetheriano, integralmente fechado, e todo ideal primo $\mathfrak{p} \neq 0$ é maximal. 
Demonstração. Vide [(NEUKIRCH, 1937), Teorema 3.1]

Definição 59. Um domínio, noetheriano, integralmente fechado no qual todo ideal primo não nulo é maximal é dito de domínio de Dedekind. Assim $\mathscr{O}_{F}$ é um domínio de Dedekind.

\subsubsection{Retículo}

Definição 60. Seja $V$ um $\mathbb{R}$-espaço vetorial de dimensão $n$. Um retículo em $V$ é um subgrupo da forma

$$
\Gamma:=\mathbb{Z} v_{1}+\mathbb{Z} v_{2}+\cdots+\mathbb{Z} v_{r}
$$

com $v_{1}, v_{2}, \cdots, v_{r}$ vetores linearmente independente em $V$. A $r$-tupla $\left(v_{1}, \cdots, v_{r}\right)$ é dita de base e o conjunto

$$
\Phi:=\left\{x_{1} v_{1}+\cdots+x_{r} v_{r}: x_{i} \in \mathbb{R}, 0 \leq x_{i}<1\right\}
$$

é dito de paralelepípedo fundamental. O retículo é dito de completo ou uma $\mathbb{Z}$-estrutura de $V$ se $\mathrm{r}=\mathrm{n}$. Para um paralelepípedo fundamental $\Phi$ de um retículo completo

$$
\Gamma=\mathbb{Z} v_{1}+\mathbb{Z} v_{2}+\cdots+\mathbb{Z} v_{n}
$$

em $V$. O covolume de $\Gamma$ é definido por

$$
R_{\Phi}:=\operatorname{Vol}(V / \Gamma)=\left|\operatorname{det}\left(v_{1}, \cdots, v_{n}\right)\right|
$$

Observação 30. Se $\Gamma:=\mathbb{Z} v_{1}^{\prime}+\mathbb{Z} v_{2}^{\prime}+\cdots+\mathbb{Z} v_{n}^{\prime}$ é outra base, então a matriz cambio de base entre $\left\{v_{i}\right\}$ e $\left\{v_{i}^{\prime}\right\}$ tem determinante \pm 1 (é invertível e com coeficientes inteiros), assim o covolume independe da escolha da base.

A completitude de um retículo é equivalente ao fato que o conjunto de todas as traslações $\Phi+\lambda, \forall \lambda \in \Gamma$ cobrem $V$. A definição de acima faz uso de uma escolha de vetores linearmente independentes, mas precisamos de uma caracterização de retículos que seja independente da escolha. Note que, um retículo é um subgrupo finitamente gerado de $V$. Pero não necessariamente cada grupo finitamente gerado é um retículo, por exemplo, $\mathbb{Z}+\mathbb{Z} \sqrt{2} \subseteq \mathbb{R}$ não é um retículo. Pero cada retículo $\Gamma=\mathbb{Z} v_{1}+\cdots+\mathbb{Z} v_{r}$ tem uma propriedade especial, ser um subgrupo discreto de $V$. Isto é, para cada ponto $\lambda \in \Gamma$ é um ponto isolado no sentido que não existe uma vizinhança que contenha outro ponto de $\Gamma$. De fato, se

$$
\lambda=a_{1} v_{1}+\cdots+a_{r} v_{r} \in \Gamma,
$$

então, estendendo $v_{1}, \cdots, v_{r}$ a uma base $v_{1}, \cdots, v_{n}$ de $V$, o conjunto

$$
\left\{x_{1} v_{1}+\cdots+x_{n} v_{n}: x_{i} \in \mathbb{R},\left|a_{i}-x_{i}\right|<1 \text { para cada } i=1, \cdots m\right\}
$$

claramente é uma vizinhança. Esta propriedade é necessária para caracterizar. 
Proposição 27. Seja $V$ um $\mathbb{R}$-espaço vetorial. Um subgrupo $\Gamma$ de $V$ é um retículo se e somente se é discreto.

Demonstração. Vide [(NEUKIRCH, 1937), Proposição 4.2]

Teorema 20 (Teorema das unidades de Dirichlet). O grupo $K_{1}\left(\mathscr{O}_{F}\right) \cong \mathscr{O}_{F}^{\times}$é finitamente gerado, mais precisamente,

$$
K_{1}\left(\mathscr{O}_{F}\right) \cong \mathscr{O}_{F}^{\times} \cong \mathbb{Z}^{r 1+r 2-1} \oplus \mu_{F},
$$

onde

. $r_{1}$ é o numero de mergulhos reais $\sigma_{1}, \cdots \sigma_{r_{1}}: F \rightarrow \mathbb{R}$

- $r_{2}$ é o numero de pares de mergulhos complexos $\sigma_{r_{1}+1}, \cdots \sigma_{r_{1}+r_{2}}, \overline{\sigma_{r_{1}+1}}, \cdots, \overline{\sigma_{r_{1}+r_{2}}}: F \rightarrow \mathbb{C}$

- $\mu_{F}$ é o grupo das unidades de $F$. Isto é, $\mu_{F}=\left\{x \in F: \exists n \in \mathbb{N}\right.$ tais que $\left.x^{n}=1\right\}$.

Demonstração. Vide [(NEUKIRCH, 1937), Teorema 7.4]

\subsection{CW-complexos}

Um espaço topológico $X$ e $f: X \longrightarrow Y$ uma função sobrejetora sobre um conjunto $Y$. Então $\tau_{Y}^{f}=\left\{U \subseteq Y \mid f^{-1}(U)\right.$ é um aberto de $\left.X\right\}$ é uma topologia sobre $Y$. Esta topologia é a mais fina sobre $Y$ tal que $f$ é continua. Tal topologia $\tau_{Y}^{f}$ é dito de topologia quociente sobre $Y$ com respeito a $f$. Um função continua sobrejetora $f: X \longrightarrow Y$ entre espaços topológicos é dita de mapa quociente se tem a seguinte propriedade: $U \subseteq Y$ é aberto se, e somente se $f^{-1}(U) \subseteq X$ é aberto. Se $f: X \longrightarrow Y$ é uma mapa quociente, então $Y$ é dito de espaço quociente de $X$.

Se $R$ é uma relação de equivalência sobre $X$, então $X / R$ denota o conjunto das classes de equivalência. A aplicação canônica $\pi: X \longrightarrow X / R$ que envia $x \in X$ na sua classe de equivalência. O espaço quociente $X / R$ é definido pelo conjunto $X / R$ junto com a topologia quociente com respeito a $\pi$.

Sejam $A \subseteq X, j: A \longrightarrow X$ a inclusão e $f: A \longrightarrow Y$ uma função continua. Nós identificamos na suma topológica $X \sqcup Y$ para cada $a \in A$ o ponto $a \in X$ com o ponto $f(a) \in Y$, isto é, nós consideramos a relação de equivalência sobre a suma topológica $X \sqcup Y$ com classe de equivalência $[z]=\{z\}$ para $z \notin A \sqcup f(A)$ e $[z]=\{z\} \cup f^{-1}(z)$ para $z \in f(A)$. O espaço quociente é denotado por $Y \cup_{f} X$ o qual é dito de espaço adjunto obtido por colagem $X$ via $f$ sobre $Y$. As inclusões canônicas $X \longrightarrow X \sqcup Y$ e $Y \longrightarrow X \sqcup Y$ induz as funções continuas $F: X \longrightarrow Y \cup_{f} X$ e $J: Y \longrightarrow$ $Y \cup_{f} X$, a função continua $J: Y \longrightarrow Y \cup_{f} X$ é um mergulho.

Proposição 28. Seja $A \subseteq X$ um subconjunto fechado. Então temos a seguinte propriedades:

(1) $J$ é um mergulho fechado. 
(2) $F$ restrita a $X \backslash A$ é um mergulho aberto.

(3) Se $X, Y$ são espaços $T_{1}$ (Resp. espaços $T_{4}$ ), então $Y \cup_{f} X$ é um espaço $T_{1}$ (Resp. espaços $\left.T_{4}\right)$.

(4) Se $f$ é uma aplicação quociente, então $F$ é uma aplicação quociente.

Demonstração. Vide [(DIECK, 2008), Proposição 1.2.4]

Usamos os subconjuntos padrão de espaços euclidianos $S^{n-1}, \mathbb{D}^{n}, E^{n}=\mathbb{D}^{n} \backslash S^{n-1},(n \geq$ 1). Nós estabeleceremos $S^{-1}=\emptyset$ e seja $\mathbb{D}^{0}$ um ponto, por tanto $E^{0}=\mathbb{D}^{0}$. Uma célula $k$ dimensional(ou uma $k$-célula) em um espaço topológico $X$ é um subconjunto $e^{k}$ o qual é, com a topologia de subespaço, homeomorfo a $E^{k}$. Um ponto é sempre uma 0-célula. Seja $X$ um espaço topológico. Para $n \geq 1$ e seja $\varphi=\left\langle\varphi_{\lambda}\right\rangle: \bigsqcup_{\lambda \in \Lambda} S_{\lambda}^{n-1} \longrightarrow X$ uma aplicação continua, onde $\bigsqcup_{\lambda \in \Lambda} S_{\lambda}^{n-1}$ é a união disjunta de esferas de dimensão $n-1, S_{\lambda}^{n-1} \subset \mathbb{D}_{\lambda}^{n}, \lambda \in \Lambda$. Definamos a $n$-ésima extensão celular de $X$ com aplicação de colagem $\varphi$ e aplicação característica $F$ como o espaço adjunto $X \cup_{\varphi}\left(\bigsqcup_{\lambda \in \Lambda} \mathbb{D}_{\lambda}^{n}\right)$. Assim a $n$-ésima extensão celular de $X$ da o seguinte diagrama comutativo

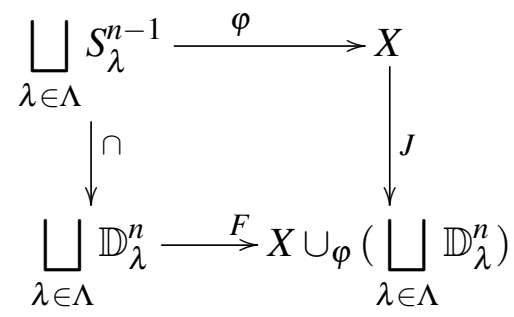

Observação 31. Como $\bigsqcup_{\lambda \in \Lambda} S_{\lambda}^{n-1}$ é um subconjunto fechado de $\bigsqcup_{\lambda \in \Lambda} \mathbb{D}_{\lambda}^{n}$. Então pela proposição anterior a $n$-ésima extensão celular de $X$ contem ao espaço $X$ como subespaço fechado e contem a $\bigsqcup_{\lambda \in \Lambda} E_{\lambda}^{n}$ como subespaço aberto. Assim as $n$-células $e_{\lambda}^{n}:=F\left(E_{\lambda}^{n}\right)$ são as componentes conexas do complementar do espaço topológico $X$.

Definição 61. Um espaço $C W$-complexo (ou um complexo Whitehead) é um espaço topológico $X$ junto com uma sequencia de subespaços $X^{0} \subseteq X^{1} \subseteq \cdots \subseteq X^{n-1} \subseteq X^{n} \subseteq \cdots \subseteq X$ tal que:

(1) $X=\bigcup_{0 \leq n} X^{n}$,

(2) O espaço topológico $X^{0}$ é discreto.

(3) Para cada $n \geq 1, X^{n}$ é homeomorfo a uma $n$-ésima extensão celular de $X^{n-1}$.

(4) a topologia de $X$ é coerente com a sequencia,isto é, um subconjunto $A \subseteq X$ é fechado se, e somente se, $A \cap X^{n}$ é um fechado em $X^{n}$ para cada . 
Um subconjunto $A \subseteq X$ de um $C W$-complexo é um subcomplexo se é a união de células e o fecho de cada célula em A esta contida em A. Seja $G$ um grupo agindo sobre um espaço topológico $X$.

- Dizemos que $G$ age livre e propriamente descontinua, se para cada $x \in X$ existe uma vizinhança $U_{x}$ de x tal que $g U \cap U=\emptyset$ para todo $g \in G-\{e\}$ (e elemento neutro do grupo $G)$.

- Dizemos que $G$ age celularmente sobre $X$, se verifica o seguinte:

(i) Para cada $g \in G$ e cada célula aberta $E_{\lambda}$ de $X$, a traslação da esquerda $g E_{\lambda}$ é uma célula aberta de $X$ da mesma dimensão.

(ii) Se $g E_{\lambda}=E_{\lambda}$, então $g=e$ ( $e$ elemento neutro do grupo $\left.G\right)$.

Observação 32. Pela definição dada cima. Seja $X$ um espaço topológico tal que $\mathrm{G}$ age celulamente sobre $X$, então $\mathrm{G}$ age livre e propriamente descontinua sobre $X$.

Lema 9. Seja $X$ um espaço topológico Hausdorff e $G$ uma ação livre e propriamente descontinua sobre $X$, então o mapa quociente $\pi: X \longrightarrow X / G$ é uma aplicação de recobrimento.

Demonstração. Primeiro provemos que $\pi$ é uma aplicação aberta. Seja $U \subset X$ um aberto. Desde que $X / G$ tem a topologia quociente, $\pi(U)$ é um aberto de $X / G$ se e somente se $\pi^{-1}(\pi(U))$ é um aberto de $X$. Da definição do $\pi$, temos

$$
\pi^{-1}(\pi(U))=\bigcup_{g \in G} g U
$$

Desde que cada $g \in G$ induz um homeomorfismo de $X, g U$ é um aberto para todo $g \in G$, e assim $\pi^{-1}(\pi(U))$ é um aberto de $X$.

Agora provemos o lema. Nós necessitamos provar que $\pi$ é uma aplicação de recobrimento. Para isto, seja $[x] \in X / G$ fixo arbitrário. Seja $U_{x}$ uma vizinhança de $x$ dada pela ação $G$ ( $G$ age livre e propriamente descontinua). Assim temos as seguintes propriedades de $\pi$ :

(i) $\pi\left(U_{x}\right)$ é uma vizinhança de $x$, pois $\pi$ é uma aplicação aberta.

(ii) $\left\{g U_{x}\right\}_{g \in G}$ é uma descomposição aberta disjunta de $\pi^{-1}\left(p\left(U_{x}\right)\right)\left(\pi^{-1}\left(\pi\left(U_{x}\right)\right)=\bigsqcup_{g \in G} g U_{x}\right)$.

(iii) $\left.\pi\right|_{g U_{x}}: g U_{x} \longrightarrow \pi\left(g U_{x}\right)=\pi\left(U_{x}\right)$ é uma aplicação continua, aberta e sobrejetora $\left(g U_{x}\right.$ é um aberto de $X)$.

Portanto, resta verificar que $\left.\pi\right|_{g U_{x}}$ é injetora. Em efeito, sejam $x_{1}, x_{2} \in U_{x}$ tal que $\pi\left(g x_{1}\right)=\pi\left(g x_{2}\right)$. Assim temos, $\left[x_{1}\right]=\left[g x_{1}\right]=\left[g x_{2}\right]=\left[x_{2}\right]$ em $X / G$. Seja $h \in G$ tal que $x_{1}=h x_{2}$ e como $x_{1} \in U_{x}$ e $h x_{2} \in h U_{x}$, o qual implica que $U_{x} \cap h U_{x}=\emptyset$, e assim $h=e$, e assim $g x_{1}=g x_{2}$. Portanto, $\pi: X \longrightarrow X / G$ é uma aplicação de recobrimento. 
Proposição 29. Seja $X$ um espaço topológico e $G$ uma grupo que age livre e propriamente descontinua sobre $X$, então:

(1) a aplicação quociente $p: X \longrightarrow X / G, p(x)=G x$, é espaço de recobrimento normal,

(2) $G$ é o grupo de transformações de Deck de seu espaço de recobrimento $p: X \longrightarrow X / G$ se $X$ é conexo por caminhos.

(3) $G$ é isomorfo a $\pi_{1}(X / G) / p_{*}\left(\pi_{1}(X)\right)$ se $X$ é conexo por caminhos e localmente conexo por caminhos.

Demonstração. Vide [(HATCHER, 2002), Proposição 1.40]

Proposição 30. Seja $p:\left(\widetilde{X}, \widetilde{x_{0}}\right) \longrightarrow\left(X, x_{0}\right)$ uma aplicação de recobrimento, então $p_{*}: \pi_{n}\left(\widetilde{X}, \widetilde{x_{0}}\right) \longrightarrow$ $\pi_{n}\left(X, x_{0}\right)$ é um isomorfismo para todo $n \geq 2$.

Demonstração. Vide [(HATCHER, 2002), Proposição 4.1]

Teorema 21 (Whitehead). Se o mapa $f: X \longrightarrow Y$ entre espaços $C W$-complexo induz isomorfismos $f_{*}: \pi_{n}(X) \longrightarrow \pi_{n}(Y)$ para todo $n \geq 1$ e bijecção para $n=0$, então $f$ é uma equivalência homotópica. No caso $f$ seja a inclusão de um subcomplexo $X \hookrightarrow Y$, a inclusão é forte: $X$ é um retrato por deformação de $Y$.

Demonstração. Vide [(HATCHER, 2002), Teorema 4.5]

A partir de agora todos os espaços topológicos terão o mesmo tipo de homotopia de um $C W$-complexo.

Teorema 22. Seja $G$ um grupo. Então existe um $C W$-complexo contráctil $\widehat{X_{G}}$ tal que $G$ age livre e celularmente sobre $\widehat{X_{G}}$ (e portanto propriamente descontinua) e $\widehat{X_{G}} / G$ é um $C W$-complexo.

Demonstração. Vide [(ROSENBERG, 1994), Teorema 5.1.15]

Corolário 2. Seja $G$ e $\widehat{X_{G}}$ como no teorema anterior, então $p: \widehat{X_{G}} \longrightarrow \widehat{X_{G}} / G$ é uma aplicação de recobrimento.

Demonstração. Segue do Teorema 22 e o Lema 9.

Definição 62 (espaço classificante). Seja $G$ um grupo. Um espaço topológico $X$ é dito de espaço classificante de $G$ ou um espaço $K(G, 1)$, se $X$ é conexo por caminhos, tem espaço de recobrimento universal e $\pi_{1}\left(X, x_{0}\right)=G$. 
O Teorema 22 garante a existência do espaço classificante para quaisquer grupo $G$, nos denotamos ao espaço classificante de $G$ por $B G$ e seu espaço de recobrimento universal por $E G$. Pelo lema 9 temos que $\pi: E G \longrightarrow B G$ é uma aplicação de recobrimento e segue da proposição 30 e do fato que $E G$ é contrátil, que :

$$
\pi_{n}\left(B G, p\left(x_{0}\right)\right) \cong \pi_{n}\left(E G, x_{0}\right)=0
$$

para todo $n \leq 2$. Além disso, pela proposição 29 temos que:

$$
G \cong \frac{\pi_{1}\left(B G, p\left(x_{0}\right)\right)}{p_{*}\left(\pi_{n}\left(E G, x_{0}\right)\right)}=\pi_{1}\left(B G, p\left(x_{0}\right)\right) .
$$

E assim temos que

$$
\pi_{n}\left(B G, p\left(x_{0}\right)\right) \cong\left\{\begin{array}{ccc}
G & \text { si } & n=1 \\
0 & \text { si } & n \neq 0
\end{array}\right.
$$

O seguinte Teorema, mostra que o tipo de homotopia do espaço classificante de $G$ é unicamente determinado por $G$.

Exemplo 12. Seja $G$ um grupo, sabemos que $G$ age livre e propriamente descontinua sobre $E G$ e o mapa $\pi: X \longrightarrow G / X$ uma aplicação de recobrimento, então pela Proposição 16 temos que

$$
\begin{gathered}
H_{n}(G, M) \cong H_{n}(B G, M), \\
H^{n}(G, M) \cong H^{n}(B G, M) .
\end{gathered}
$$

onde $M$ é um $G$-modulo trivial. Em particular

$$
\begin{aligned}
H_{n}(G, \mathbb{Z}) \cong H_{n}(B G, \mathbb{Z}), \\
H^{n}(G, \mathbb{Z}) \cong H^{n}(B G, \mathbb{Z}),
\end{aligned}
$$

onde $\mathbb{Z}$ é visto como G-modulo trivial.

Proposição 31. Seja $X$ um $C W$-complexo conexo por caminhos e seja $Y$ o espaço classificante de $G$. Então todo homomorfismo $\varphi: \pi_{1}\left(X, x_{0}\right) \longrightarrow \pi_{1}\left(Y, y_{0}\right)$ é induzido por um mapa $f:\left(X, x_{0}\right) \longrightarrow$ $\left(Y, y_{0}\right)$, isto é $\varphi=f_{*}$, que é único a menos de homotopia fixando $x_{0}$.

Demonstração. Vide [(HATCHER, 2002), Proposição 1B.9]

Observação 33. Se $X$ é um $C W$-complexo conexo por caminhos tais que $\pi_{1}\left(X, x_{0}\right)=G$ e $\pi_{n}\left(X, x_{0}\right)=0$ para $n>1$, para algum $x_{0} \in X$, então pela Proposição 30 e o Teorema 21, o espaço de recobrimento universal de $X$ tem todos seus grupos de homotopia triviais e por tanto é contrátil. E segue da Proposição 31 que o tipo de homotopia do espaço classificante é unicamente determinado por $G$. 


\section{5 t-construção}

Nesta secção, daremos a +-construção, o qual é o resultado da colagem de células para modificar o grupo fundamental, sem modificar os grupos de homologia.

Definição 63. Seja $X$ um espaço topológico, o grupoide fundamental de $X$ é a categoria denotada por $\pi(X)$ onde:

1. $\mathrm{Ob}(\pi(X)):=X$

2. $\operatorname{Hom}(x, y):=\{[\alpha]: \alpha:[0,1] \longrightarrow X$ tais que $\alpha(0)=x, \alpha(1)=y\}$, onde $[\alpha]$ representa a classe de homotopia de $\alpha$.

Observação 34. 1. Como a definição sugere, isto é um grupoide, que é uma categoria a qual todo morfismo são isomorfismo. Para ver a inversa do $[\alpha]$ só basta tomar a classe de homotopia do caminho inverso $\alpha^{-1}$.

2. Além disso temos que $\operatorname{Hom}(x, x)=\pi(X, x)$, e assim podemos entender esta categoria como simplesmente uma maneira de organizar todos os grupos fundamentais de $X$ e todos os isomorfismos entre eles induzidos por caminhos.

Definição 64. Seja $X$ um espaço topológico, um sistema de coeficientes locais sobre $X$ é um functor contravariante

$$
\mathscr{H}: \pi(X) \longrightarrow \mathrm{Gr}_{\mathrm{Ab}}
$$

do grupoide fundamental de $X$ na categoria de grupos abelianos.

Observação 35. Se $X$ é um espaço conexo por caminhos com ponto base $x_{0} \in X$, então ter uma ação de um grupo $A$ sobre $\pi_{1}\left(X, x_{0}\right)$ sobre $G$ é equivalente a pedir um sistema de coeficientes locais para $X$.

Exemplo 13. 1. Sejam $X$ um espaço topológico e $A$ um grupo abeliano. Definimos $\mathscr{H}$ : $\pi(X) \longrightarrow \mathrm{Gr}_{\mathrm{Ab}}$ por $\mathscr{H}(x)=A$ e $\mathscr{H}([\alpha])=I d_{A}$ para todo caminho $\alpha$. Este é o sistema de coeficiente locais constante, o qual denotaremos só por $A$.

2. Seja $X$ um espaço topológico, definimos $\mathscr{H}: \pi(X) \longrightarrow \mathrm{Gr}_{\mathrm{Ab}}$ por

$$
\begin{aligned}
\mathscr{H}(x) & =\pi(X, x) \\
\mathscr{H}([\alpha]) & :=\alpha_{\#}: \pi_{1}(X, \alpha(1)) \longrightarrow \pi_{1}(X, \alpha(0))
\end{aligned}
$$

onde $\alpha_{\sharp}$ é o mapa definido por:

$$
\alpha_{\#}([\lambda])=\left[\alpha * \lambda * \alpha^{-1}\right]
$$


onde se pode mostrar facilmente que o homomorfismo $\alpha_{\#}$ independe do representante $\lambda$ e depende só da classe de homotopia de $\alpha$, e por tanto é um isomorfismo com inversa $\left(\alpha^{-1}\right) \#$.

3. Sejam $X, Y$ espaços topológicos e $f: X \longrightarrow Y$ uma aplicação continua, se $\mathscr{H}$ é um sistema de parâmetros locais para $Y$ então o mapa $f$ induz um sistema de parâmetros $f^{*}(G): \pi(X) \longrightarrow \mathrm{Gr}_{\mathrm{Ab}}$ sobre $X$, dado por:

$$
\begin{aligned}
\left(f^{*} \mathscr{H}\right)(x) & :=\mathscr{H}(f(x)) \\
\left(f^{*} \mathscr{H}\right)([\alpha]) & :=\mathscr{H}([\alpha \circ f]), \text { para todo morfismo }[\alpha] \text { em } \pi(Y) .
\end{aligned}
$$

Definição 65. Sejam $X$ um espaço topológico e $\mathscr{H}$ um sistema de coeficientes locais de $X$. Definimos

$$
C_{n}(X, \mathscr{H})=\left\{\sum g_{i} \otimes \sigma_{i}: \sigma_{i}: \Delta^{n} \longrightarrow X, g_{i} \in \mathscr{H}\left(\sigma_{i}\left(e_{0}\right)\right)\right\} \subseteq \bigoplus_{x \in X} \mathscr{H}(x) \otimes_{\mathbb{Z}} C_{n}(X)
$$

$\mathrm{e}$

$$
\partial_{n}: C_{n}(X, \mathscr{H}) \longrightarrow C_{n-1}(X, \mathscr{H})
$$

dado por

$$
\partial_{n}(g \otimes \sigma)=\sum_{i=1}^{n}(-1)^{i} g \otimes \delta_{i} \sigma+\mathscr{H}\left(\lambda_{\sigma}\right)(g) \otimes \delta_{0} \sigma .
$$

Teorema 23 (Quillen). Seja $X$ um $C W$-complexo conexo por caminhos, $N$ um subgrupo normal perfeito de $\pi_{1}(X, x)$. Então existe um $C W$-complexo $X^{+}$(dependendo de $N$ ), dita de + -construção relativa a $N$ e um mapa $i:(X, x) \longrightarrow\left(X^{+}, x^{+}\right)$tais que:

(i) existe uma sequencia exata curta

$$
0 \longrightarrow N \longrightarrow \pi_{1}(X, x) \stackrel{f_{*}}{\longrightarrow} \pi_{1}\left(X^{+}, x^{+}\right) \longrightarrow 0
$$

(ii) para cada sistema de coeficientes locais $\mathscr{H}$ de $X^{+}$,

$$
f_{*}: H_{n}\left(X, f^{*} \mathscr{H}\right) \longrightarrow H_{n}\left(X^{+}, \mathscr{H}\right)
$$

é um isomorfismo para todo $n \geq 0$,

(iii) se $g:(X, x) \longrightarrow(Y, y)$ é uma aplicação continua tais que

$$
N \subset \operatorname{ker}\left(g_{*}: \pi_{1}(X, x) \longrightarrow \pi_{1}(Y, y)\right),
$$

então existe uma aplicação continua $h:\left(X^{+}, x^{+}\right) \longrightarrow(Y, y)$, única a menos de homotopia, tais que o diagrama

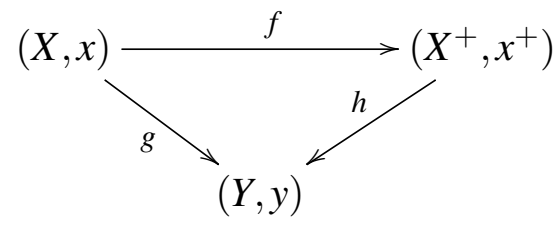

é comutativo. 
Demonstração. Vide [(SRINIVAS, 1991), Teorema 2.1]

Observação 36. 1. A construção de $X^{+}$é por colagem de 2-células e 3-células a $X$, e assim $X^{+}$é um par- $C W$ (relativo) de dimensão 3 , tomamos $x^{+}=x$.

2. Tomando $\mathbb{Z}$ o sistema de coeficientes locais constante sobre $X^{+}$, entao o sistema de coeficientes locais $f^{*} \mathbb{Z}$ induzido pela $f$ é $\mathbb{Z}$. E assim pelo item (ii) temos que

$$
H_{n}(X, \mathbb{Z}) \cong H_{n}\left(X^{+}, \mathbb{Z}\right),
$$

para todo $n \geq 0$, isto quer dizer que $X$ e $X^{+}$tem o mesmo grupo de homologia. Além disso, o item (iii) caracteriza $X^{+}$ao menos de homotopia.

Proposição 32. Seja $p:(\widehat{X}, \widehat{x}) \longrightarrow(X, x)$ o espaço de recobrimento de $X$ correspondente ao subgrupo normal perfeito $N \triangleleft \pi_{1}(X, x)$, e seja $\left(\widetilde{X}^{+}, \widetilde{x}^{+}\right)$o universal espaço de recobrimento de $\left(X^{+}, x^{+}\right)$. Então $\left(\widetilde{X}^{+}, \widetilde{x}^{+}\right)$é, a menos de homotopia, o resultado de aplicar a +-construção de $(\widehat{X}, \widehat{x})$ relativa a $N$.

Demonstração. Vide [(SRINIVAS, 1991), Proposição 2.3]

Proposição 33. Se $f_{i}:\left(X_{i}, x_{i}\right) \longrightarrow\left(\widehat{X}_{i}^{+}, \widehat{x}_{i}^{+}\right), i=1,2$ são obtidos da +-construção de $X_{i}$ relativa ao subgrupo normal perfeito $N_{i} \triangleleft \pi_{i}\left(X_{i}, x_{i}\right)$. Então $\left(f_{1}, f_{2}\right):\left(X_{1} \times X_{2},\left(x_{1}, x_{2}\right)\right) \longrightarrow\left(X_{1}^{+} \times\right.$ $\left.X_{2}^{+},\left(x_{1}^{+}, x_{2}^{+}\right)\right)$é, a menos de homotopia, o resultado de aplicar a + -construção a $X_{1} \times X_{2}$ relativa a $N_{1} \times N_{2} \triangleleft \pi_{1}\left(X_{1} \times X_{2},\left(x_{1}, x_{2}\right)\right)$.

Demonstração. Para $X_{i}$ temos uma sequencia exata curta

$$
0 \longrightarrow N_{i} \longrightarrow \pi_{1}\left(X_{i}, x_{i}\right) \stackrel{\left(f_{i}\right)_{*}}{\longrightarrow} \pi_{1}\left(X_{i}^{+}, x_{i}^{+}\right) \longrightarrow 0
$$

para cada $i=1,2$, e assim temos a sequência exata

$$
0 \longrightarrow N_{1} \times N_{2} \longrightarrow \pi_{1}\left(X_{1} \times X_{2},\left(x_{1}, x_{2}\right)\right) \stackrel{\left(f_{1} \times f_{2}\right)_{*}}{\longrightarrow} \pi\left(X_{1}^{+} \times X_{2}^{+},\left(x_{1}^{+}, x_{2}^{+}\right)\right) \longrightarrow 0
$$

e assim se verifica (i) do Teorema 23. Por um argumento similar pode-se provar (ii). E (iii), caracteriza o tipo de homotopia do espaço resultante.

Exemplo 14. Sejam $R$ um anel com unidade e $X=B G L(R)$, o espaço classificante do grupo linear geral infinito $G L(R)$. Pela Proposição 22 temos que $E(R) \triangleleft G L(R)=\pi_{1}\left(B G L(R), x_{0}\right)$ é um subgrupo normal perfeito. Assim pelo Teorema 23 existe um $C W$-complexo $B G L(R)^{+}$, a +-construção de $B G L(R)$ relativo a $E(R)$, e assim temos que:

$$
H_{n}\left(B G L(R)^{+}, \mathbb{Z}\right) \cong H_{n}(B G L(R), \mathbb{Z}) \cong H_{n}(G L(R), \mathbb{Z}) .
$$

Seja $\widehat{B G L(R)}$ o espaço de recobrimento de $B G L(R)$ correspondente a $E(R)$, isto é $\pi_{1}(\widehat{B G L(R)})=$ $E(R)$, segue da proposição 30 que $\widehat{B G L(R)}$ é o espaço classificante de $E(R)$, isto é $B E(R)=$ $\widehat{B G L(R)}$. E segue da proposição 32 que $B E(R)^{+}$é o espaço de recobrimento universal de $B G L(R)^{+}$. 


\subsection{K-teoria de Quillen}

Nesta secção, generalizaremos a definição dos grupos $K_{0}$ e $K_{1}$ e definiremos os $K$-grupos de ordem superior dado por Quillen.

Definição 66. Sejam $R$ um anel com unidade e $K_{0}(R)$ o grupo de Grotendieck. Definimos para cada $n \geq 0$, o $n$-ésimo $K$-grupo do anel $R$ como sendo o $n$-ésimo grupo de homotopia do espaço $B G L(R)^{+} \times K_{0}(R)$, onde $B G L(R)^{+}$é a +-construção de $B G L(R)$ relativa a $E(R)$ e $K_{0}(R)$ é considerado um espaço topológico discreto, isto é

$$
K_{n}^{Q}(R):=\pi_{n}\left(B G L(R)^{+} \times K_{0}(R)\right)
$$

Segue da definição que

$$
K_{0}^{Q}(R)=\pi_{0}\left(B G L(R)^{+} \times K_{0}(R)\right) \cong \pi_{0}\left(K_{0}(R)\right)=K_{0}(R)
$$

e pelo Teorema 23 (Quillen) temos

$$
K_{1}^{Q}(R) \cong \pi_{1}\left(B G L(R)^{+}\right) \cong G L(R) /[G L(R), G L(R)]=K_{1}(R),
$$

e assim, vemos que a definição de Quillen generaliza os grupos $K_{0}$ e $K_{1}$ de $R$ definidos anteriormente. 
CAPÍTULO

\section{4}

REGULADOR DE BOREL

Neste capitulo, daremos a definição de um regulador e apresentaremos o Teorema de Borel. Além disso, daremos um teorema para poder calcular o posto do $K$-grupos para o anel de inteiros de um corpo numérico.

\subsection{Definição do Regulador de Borel}

Seja $F$ um corpo numérico, isto é uma extensão de corpo finita dos números racionais $\mathbb{Q}$, e seja $\mathscr{O}_{F}$ seu anel de inteiros. Para cada $m \geq 2$, o mapa regulador do Borel é um homomorfismo

$$
K_{m}\left(\mathscr{O}_{F}\right) \longrightarrow V_{m}
$$

do $m$-ésimo $K$-grupo do anel $\mathscr{O}_{F}$ a algum $\mathbb{R}$-espaço vetorial.

Para o caso $R=\mathscr{O}_{F}$, segue do Teorema de Bass-Milnor-Serre que $\operatorname{SL}\left(\mathscr{O}_{F}\right)=E\left(\mathscr{O}_{F}\right)$. Além disso, pelo Exemplo 14 nos temos que $B S L\left(\mathscr{O}_{F}\right)^{+}$é o espaço de recobrimento universal de $B G L\left(\mathscr{O}_{F}\right)^{+}$e assim nos temos

$$
\pi_{m}\left(B S L\left(\mathscr{O}_{F}\right)^{+}\right) \cong \pi_{m}\left(B G L\left(\mathscr{O}_{F}\right)^{+}\right), \forall m \geq 2
$$

Por tanto o morfismo de Hurewicz e o Teorema de Cartan-Serre induz o isomorfismo

$$
h_{\mathbb{Q}}: K_{m}\left(\mathscr{O}_{F}\right) \otimes_{\mathbb{Z}} \mathbb{Q} \longrightarrow P_{m}\left(S L\left(\mathscr{O}_{F}\right), \mathbb{Q}\right), \text { para cada } m \geq 2 .
$$

Seja $G$ um $\mathbb{Q}$-grupo algebraico afim. Assumimos que $G(\mathbb{R})$ é um grupo de Lie conexo. Seja $\Gamma$ um grupo discreto, quaisquer homomorfismo de grupos $\varphi: \Gamma \longrightarrow G(\mathbb{R})$ induz um morfismo

$$
\varphi^{*}: H_{\text {cont }}^{*}(G(\mathbb{R}), \mathbb{R}) \longrightarrow H^{*}(\Gamma, \mathbb{R})=H_{\text {cont }}^{*}(\Gamma, \mathbb{R})
$$

Além disso, nos construímos o isomorfismo $\gamma^{\prime}$ dado em (2.74), isto é

$$
\gamma^{\prime}: H_{D R}^{*}(C T(G(\mathbb{R}))) \longrightarrow H_{\text {cont }}^{*}(G(\mathbb{R}), \mathbb{R})
$$


Assim, obtemos um morfismo

$$
j:=\varphi^{*} \circ \gamma^{\prime}: H_{D R}^{*}(C T(G(\mathbb{R})), \mathbb{R}) \longrightarrow H^{*}(\Gamma, \mathbb{R})
$$

pela construção este morfismo é uma morfismo de álgebras e pela compacidade da $C T(G(\mathbb{R}))$, nos podemos dualizar $j$, o dual sera denotado por $j^{\vee}$, isto é

$$
j^{\vee}: H_{*}(\Gamma, \mathbb{R}) \longrightarrow H_{*}(C T(G(\mathbb{R})), \mathbb{R}) .
$$

Desde que $j$ é um morfismo de álgebras, $j^{\vee}$ é um morfismo de coálgebras. Por tanto induz um morfismo, denotado por $j^{\vee}$, entre os subespaços primitivos na homologia

$$
j^{\vee}: P_{*}(\Gamma, \mathbb{R}) \longrightarrow P_{*}(C T(G(\mathbb{R})), \mathbb{R}) .
$$

Para cada $n \geq 1$ denotamos $G_{n}:=\operatorname{Res}_{F / \mathbb{Q}} S L_{n, F} \mathbf{O} \mathbb{Q}$-grupo algebraico afim, isto é, nos vemos a $S L_{n, F}$ como um $\mathbb{Q}$-grupo algebraico afim. O subgrupo $S L_{n}\left(\mathscr{O}_{F}\right)$ é um subgrupo discreto de $G_{n}(\mathbb{R})$. Seja

$$
j_{n}^{\vee}: H_{*}\left(S L_{n}\left(\mathscr{O}_{F}\right), \mathbb{R}\right) \longrightarrow H_{*}\left(C T\left(G_{n}(\mathbb{R})\right), \mathbb{R}\right)
$$

Denotamos por

$$
\begin{aligned}
G(\mathbb{R}) & =\underset{\longrightarrow}{\lim G_{n}(\mathbb{R}),} \\
C T(G(\mathbb{R})) & =\underset{\longrightarrow}{\lim C T}\left(G_{n}(\mathbb{R})\right) .
\end{aligned}
$$

assim, passando limite a (4.9), obtemos o morfismo

$$
j^{\vee}: H_{*}\left(S L\left(\mathscr{O}_{F}\right), \mathbb{R}\right) \longrightarrow H_{*}\left(C T\left(G_{n}(\mathbb{R})\right), \mathbb{R}\right)
$$

Definição 67. Seja $m \geq 2$. Definimos o $m$-ésimo mapa regulador de Borel, denotado por $r_{B o}^{\prime}$, definido por

$$
\stackrel{\mathrm{r}_{\mathrm{Bo}}^{\prime}}{\prime}: K_{m}\left(\mathscr{O}_{F}\right) \otimes \mathbb{R} \stackrel{\text { Hur }}{\longrightarrow} P_{m}\left(S L_{n}(G(\mathbb{R})), \mathbb{R}\right) \stackrel{j^{\vee}}{\longrightarrow} P_{m}(C T(G(\mathbb{R})), \mathbb{R})
$$

Teorema 24. Seja $e=\max (1, \operatorname{sdim})$. Então $H_{m}\left(G L_{n}(R), \mathbb{Z}\right) \longrightarrow H_{m}\left(G L_{n+1}(R), \mathbb{Z}\right)$ e $H_{m}\left(E_{n}(R), \mathbb{Z}\right) \longrightarrow$ $H_{m}\left(E_{n+1}(R), \mathbb{Z}\right)$ são sobrejetoras para $n \geq 2 m+e-1$, injetoras para $n \geq 2 m+e(m \geq 0)$.

Demonstração. Vide [(KALLEN, 1980), Teorema 4.11]

No caso de $R$ ser um domínio de Dedekind, temos que $e=1$. E assim podemos dar a seguinte definição

Definição 68. Sejam $n, m$ inteiros positivos. Dizemos que $n, m$ esta no rango estável se $n$ é impar e $m \geq(n-1) / 2$. 
Segue do Teorema 24, a condição $m \geq(n-1) / 2$ assegura que os morfismos

$$
\begin{array}{r}
H_{m}\left(S L_{n}\left(\mathscr{O}_{F}\right), \mathbb{Q}\right) \longrightarrow H_{m}\left(S L\left(\mathscr{O}_{F}\right), \mathbb{Q}\right), \\
H^{m}(S L(\mathbb{C}), \mathbb{R}) \longrightarrow H^{m}\left(S L_{n}(\mathbb{C}), \mathbb{R}\right),
\end{array}
$$

e o correspondente morfismo para $S L_{n}(\mathbb{R})$ são isomorfismos.

Observação 37. A condição $n$ impar na definição anterior, implica que a inclusão $\mathfrak{s o}_{n}(\mathbb{R}) \longrightarrow \mathfrak{u}_{n}$ é noncohomologus to zero. Além disso, pela Proposição 20 temos que

$$
H^{*}\left(\mathfrak{u}_{n}, \mathfrak{s o}_{n}(\mathbb{R})\right) \longrightarrow H^{*}\left(\mathfrak{u}_{n}\right)
$$

é injectiva.

Para obter informação sobre o mapa regulador de Borel, nos precisamos estudar o morfismo $j^{\vee}$. Isto é proporcionado pela Teoria de Borel dos grupos aritméticos. Seja $G$ um subgrupo de $G L(V)$, onde $V$ é um $\mathbb{Q}$-espaço vetorial de dimensão finita. Seja $L$ um retículo de $V$ . Denotamos por $G_{L}$ ao subgrupo de $G(\mathbb{Q})$ que deixa a $L$ fixo, isto é

$$
G_{L}:=\{g \in G(\mathbb{Q}): g(L)=L\}
$$

Definição 69. Um subgrupo $\Gamma$ de $G(\mathbb{Q})$ é dito aritmético se é comensurável, isto é, se $\Gamma \cap G_{L}$ tem índice finito em $\Gamma$ e em $G_{L}$.

Teorema 25 (Borel). Seja $G$ um semisimples $\mathbb{Q}$-grupo algebraico e seja $\Gamma$ um subgrupo aritmético de $G(\mathbb{R})$, com $\varphi: \Gamma \longrightarrow G(\mathbb{R})$ a inclusão. Seja $C T(G(\mathbb{R}))$ o gêmeo compacto de $G(\mathbb{R})$ e $j$ como em (4.6). Então existe um numero $\rho(G)$ tais que

$$
j: H^{m}(C T(G(\mathbb{R})), \mathbb{R}) \longrightarrow H^{m}(\Gamma, \mathbb{R}) .
$$

é um isomorfismo para $m \geq \rho(G)$. Esse numero $\rho(G)$ só depende da estrutura algébrica de $G$.

Demonstração. Ver[Stable real cohomology of arithmetic groups]

Observação 38. Para qualquer álgebra de Lie $G$, o numero $\rho(G)$ pode ser pequeno. Por exemplo $\rho\left(S L_{n}\right)=n / 4$. Mais, no caso de interesse $G_{n}(\mathbb{R})=S L_{n}(\mathbb{R})$, quando fazemos tender $n$ ao infinito, $\rho\left(G_{n}\right)$ também vai para o infinito.

O seguinte Corolário segue da observação anterior e da estabilidade dos grupos de cohomologia real dos grupos aritméticos.

Corolário 3. Seja $m \geq 2$ um numero inteiro. Então o mapa regulador de Borel induz um isomorfismo

$$
\mathrm{r}_{\mathrm{Bo}}^{\prime}: K_{m}\left(\mathscr{O}_{F}\right) \otimes_{\mathbb{Z}} \mathbb{R} \longrightarrow P_{m}(C T(G(\mathbb{R})) ; \mathbb{R})
$$




\subsection{O posto do grupo $K_{m}\left(\mathscr{O}_{F}\right)$}

Sejam $F$ um corpo numérico e $\mathscr{O}_{F}$ seu anel de inteiros. Seja $\Sigma$ o conjunto de imersões complexas de $F$, seja $\Upsilon$ o conjunto de archimedean places de $F$. Denotaremos $\Upsilon_{\mathbb{R}}$ ao conjunto de places correspondentes as imersões reais e $\Upsilon_{\mathbb{C}}$ ao conjuntos de places correspondentes as imersões complexas. Seja $d=[F: \mathbb{Q}]$, como é usual, denotamos $r_{1}=\# \Upsilon_{\mathbb{R}}$ e $r_{2}=\# \Upsilon_{\mathbb{C}}$. Então $d=r_{1}+2 r_{2}$. Para um $m$ fixo escolhemos um inteiro $n$ tais que $m, n$ estão no rango estável.

Seja $G_{n}=\operatorname{Res}_{F / \mathbb{Q}} S L_{n, \mathbb{F}}$, então pelo Exemplo ?? o grupo de Lie $G_{n}(\mathbb{R})$ é dado por $G_{n}(\mathbb{R})=\Pi_{v \in \Upsilon_{\mathbb{R}}} S L_{n}(\mathbb{R}) \times \Pi_{v \in \Upsilon_{\mathbb{C}}} S L_{n}(\mathbb{C})$. Pela construção do gêmeo compacto, podemos fazer componente a componente. Para o grupo $S L_{n}(\mathbb{R})$ temos que o subgrupo compacto maximal é $S O_{n}(\mathbb{R})$. Sua complexificação é $S L_{n}(\mathbb{C})$ e seu subgrupo compacto maximal é $S U_{n}$. Por tanto, o gêmeo compacto é $C T\left(S L_{n}(\mathbb{R})\right)=S O_{n}(\mathbb{R}) / S U_{n}$. Para o grupo $S L_{n}(\mathbb{C})$ o subgrupo compacto maximal é $S U_{n}$. A complexificação é $S L_{n}(\mathbb{C}) \times S L_{n}(\mathbb{C})$, com a inclusão

$$
\begin{gathered}
S L_{n}(\mathbb{C}) \longrightarrow S L_{n}(\mathbb{C}) \times S L_{n}(\mathbb{C}) \\
M \longmapsto(\bar{M}, M)
\end{gathered}
$$

A conjugação complexa é dada por

$$
\begin{gathered}
\tau: S L_{n}(\mathbb{C}) \times S L_{n}(\mathbb{C}) \longrightarrow S L_{n}(\mathbb{C}) \times S L_{n}(\mathbb{C}) \\
(M, N) \longmapsto(\bar{N}, \bar{M}) .
\end{gathered}
$$

Assim o subgrupo compacto maximal da complexificação é $S U_{n} \times S U_{n}$ e o gêmeo compacto é homeomorfo a $S U_{n}$. Por tanto, tomando limite direito, o gêmeo compacto $C T(G(\mathbb{R}))$ é homeomorfo a $(S U / S O(\mathbb{R}))^{r_{1}} \times S U^{r_{2}}$.

Teorema 26 (Borel). O posto do grupo $K_{m}\left(\mathscr{O}_{F}\right)$ para $m \geq 2$ é dado por

$$
\operatorname{rk}\left(K_{m}\left(\mathscr{O}_{F}\right)\right)=\left\{\begin{array}{llll}
0 & \text {, se } & m \equiv 0 & \bmod 2 \\
r_{1}+r_{2} & \text {, se } & m \equiv 1 & \bmod 4 \\
r_{2} & \text {, se } & m \equiv 3 & \bmod 4
\end{array}\right.
$$

Demonstração. Pelo Corolário 3, temos que

$$
\operatorname{rk}\left(K_{m}\left(\mathscr{O}_{F}\right)\right)=\operatorname{dim}_{\mathbb{R}} P_{m}(C T(G(\mathbb{R})), \mathbb{R})
$$

e pela dualidade temos que

$$
\operatorname{dim}_{\mathbb{R}} P_{m}(C T(G(\mathbb{R})), \mathbb{R})=\operatorname{dim}_{\mathbb{R}} Q^{m}(C T(G(\mathbb{R})), \mathbb{R})
$$

Além disso, pela Proposição 14 temos

$$
H^{*}(S U / S O(\mathbb{R}), \mathbb{R}) \cong \Lambda\left(x_{5}, x_{9}, \cdots, x_{4 k+1}, \cdots\right)
$$


e pelo Exemplo 18 temos

$$
H^{*}(S U, \mathbb{R}) \cong \Lambda\left(x_{3}, x_{5}, \cdots, x_{2 k+1}, \cdots\right)
$$

assim, pela formula de Kunneth, temos

$$
H^{*}(C T(G(\mathbb{R}))) \cong \Lambda\left(x_{5}, x_{9}, \cdots, x_{4 k+1}, \cdots\right)^{\otimes r_{1}} \otimes \Lambda\left(x_{3}, x_{5}, \cdots, x_{2 k+1}, \cdots\right)^{\otimes r_{2}}
$$

e assim temos

$$
\operatorname{dim}_{\mathbb{R}} Q^{m}(C T(G(\mathbb{R})), \mathbb{R})=\left\{\begin{array}{llll}
0 & \text {, se } & m \equiv 0 & \bmod 2 \\
r_{1}+r_{2} & \text {, se } & m \equiv 1 & \bmod 4 \\
r_{2} & \text {, se } & m \equiv 3 & \bmod 4
\end{array}\right.
$$

\subsection{Os valores da Função Zeta de Dedekind}

Nesta secção daremos o Teorema do Borel, o qual relaciona o regulador de Borel com os valores da função zeta de Dedekind. Uma das principais razones para usar $P_{2 n-1}(C T(G(\mathbb{R})), \mathbb{R})$ como objeto no mapa regulador de Borel é que tem uma estrutura integral natural. Desde que a $K$-teoria é definida usando homotopia, [(LICHTENBAUM, 1973)] propôs usar o retículo dado pela imagem de $\pi_{2 n-1}(C T(G(\mathbb{R})), e)$ sob o morfismo de Hurewicz. Denotamos por $L_{2 n-1}^{\prime}$ este retículo, isto é $L_{2 n-1}^{\prime}:=\mathrm{h}\left(\pi_{2 n-1}(C T(G(\mathbb{R})), e)\right)$.

A função Zeta de Riemann $\zeta(s)=\sum_{k=1}^{\infty} \frac{1}{k^{s}}$ é associada com o corpo $\mathbb{Q}$. Ela se generaliza da seguinte maneira para um corpo numérico qualquer $F$ de grau $n=[F: \mathbb{Q}]$.

Definição 70. A função zeta de Dedekind do corpo numérico $F$ é definida pela serie

$$
\zeta_{F}(s)=\sum_{\mathfrak{a} \subseteq \mathscr{O}_{F}} \frac{1}{\left[\mathscr{O}_{F}: \mathfrak{a}\right]^{s}}
$$

onde $\left[\mathscr{O}_{F}: \mathfrak{a}\right]$ é o índice de a sobre $\mathscr{O}_{F}$, o qual denotaremos por $N_{F \mid \mathbb{Q}}(\mathfrak{a})$ e é dita de norma absoluta.

Proposição 34. A serie $\zeta_{F}(s)$ converge absolutamente e uniformemente no domínio $\operatorname{Re}(s) \geq$ $1+\delta$ para cada $\delta>0$, temos que

$$
\zeta_{F}(s)=\prod_{\mathfrak{p}} \frac{1}{1-N_{F \mid \mathbb{Q}}(\mathfrak{p})^{-s}},
$$

onde $\mathfrak{p}$ varia nos ideais primos de $\mathscr{O}_{F}$. 
Pelo Corolário 3 , temos que $\mathrm{r}_{B o}^{\prime}\left(K_{2 n-1}\left(\mathscr{O}_{F}\right)\right)$ é um retículo em $P_{2 n-1}(C T(G(\mathbb{R})), \mathbb{R})$. Agora vamos a definir o regulador de Borel.

Definição 71. O regulador de Borel, $R_{B o, n}^{\prime}$, é o covolume do retículo

$$
\mathrm{r}_{B o}^{\prime}\left(K_{2 n-1}\left(\mathscr{O}_{F}\right)\right) \subset P_{2 n-1}(C T(G(\mathbb{R})), \mathbb{R}),
$$

com respeito ao retículo $L_{2 n-1}^{\prime}$.

Definição 72. Sejam $x, y \in \mathbb{R}$, escrevemos $x \sim y$ se existe $q \in \mathbb{Q}-\{0\} \operatorname{com} x=q y$.

Teorema 27 (Borel). Seja $F$ um corpo numérico. Então, para cada $n \geq 2$ temos

$$
R_{B o, n}^{\prime} \sim \pi^{-d_{n}} \lim _{s \rightarrow-n+1} \zeta_{F}(s)(s+n-1)^{-d_{n}},
$$

onde $d_{n}=\operatorname{rk}\left(K_{2 n-1}(\mathscr{O})_{F}\right)=\operatorname{dim}_{\mathbb{R}}\left(P_{2 n-1}(C T(G(\mathbb{R}))), \mathbb{R}\right)$.

Demonstração. Vide [(WEIL, 1995); Capitulo VII, Teorema 3]. 


\section{REFERÊNCIAS}

ALTMAN, A.; KLEIMAN, S. A Term of Commutative Algebra. [S.1.]: Worldwide Center of Mathematics, 2012. Citado nas páginas 21, 23 e 24.

ARTIN, E. Geometric Algebra. New York: Interscience Publishers, INC., 1957. Citado na página 67.

BASS, A. O. K. H.; PEDRINI, C. Algebraic K-theory and its applications. Singapure: World scientific publishing, 1997. Citado na página 51.

BOREL, A. Cohomologie de $s l_{n}$ et valeurs de fonctions zeta aux points entiers. Annali della Scuola Normale Superiore di Pisa, v. 4, p. 613-636, 1977. Citado na página 18.

DIECK, T. tom. Algebraic Topology. Zürich: European Mathematical Society, 2008. Citado nas páginas 73, 93 e 95 .

FOMENKO, A.; FUCHS, D. Homotopical Topology. New York: Springer-Verlag, 2016. Citado na página 51.

GREUB, S. H. W.; VANSTONE, R. Connections, curvature and cohomology. Volume II. New York and London: Academic Press, 1973. Citado nas páginas 47 e 56.

Connections, curvature and cohomology. Volume III. New York and London: Academic Press, 1976. Citado na página 60.

GUICHARDET, A. Cohomologie des groupes topologiques et des algèbres de Lie. Paris: CEDIC, 1980. Citado nas páginas 51 e 52.

HATCHER, A. Algebraic Topology. Cambridge: Cambridge University Press, 2002. Citado nas páginas 75 e 76 .

HILGERT, J.; NEEB, K.-H. Structure and Geometry of Lie Groups. New York: SpringerVerlag, 2010. Citado nas páginas 57 e 58.

KALLEN, W. van der. Homology stability for linear groups. Inventiones math, v. 60, p. 269295, 1980. Citado na página 82.

LICHTENBAUM, S. Values of zeta functions, étale cohomology, and algebraic $k$-theory. Lecture Notes in Math, v. 342, p. 489-501, 1973. Citado nas páginas 17 e 85.

MAY, J. P. A Concise Course in Algebraic Topology. Chicago: University Of Chicago Press, 1999. Citado na página 94.

MILNE, J. S. Basic Theory of Affine Group Schemes. [S.1.: s.n.], 2012. Citado na página 91. MILNOR, J. Introduction to Algebraic K-Theory. New Jersey: Princeton University Press, 1971. Citado na página 67. 
MILNOR, J. W.; MOORE, J. C. On the structure of hopf algebras. preprint, p. 1-36, 1959. Citado nas páginas 32, 33 e 35.

. On the structure of hopf algebras. The Annals of Mathematics, v. 81, n. 2, p. 211-264, 1965. Citado nas páginas 35 e 36.

MIMURA, M.; TODA, H. Topology of Lie Groups I and II. United States of America: American Mathematical Society, 1991. Citado na página 49.

NEUKIRCH, J. Algebraic Number Theory. New York: Springer-Verlag, 1937. Citado nas páginas $17,68,70,71,72$ e 85 .

ROSENBERG, J. Algebraic K-theory and its applications. New York: Springer-Verlag, 1994. Citado na página 75.

ROTMAN, J. J. An Introduction to Algebraic Topology. New York: Springer-Verlag, 1988. Citado na página 49.

SAMELSON, H. A connection between the whitehead and the pontryagin product. The Annals of Mathematics, v. 75, n. 4, p. 744-752, 1953. Citado nas páginas 50 e 51.

SRINIVAS, V. Algebraic K-theory. Boston: Birkhäuser, 1991. Citado na página 79.

WARNER, F. W. Foundations of differentiable manifolds and Lie groups. New York: Springer-Verlag, 1983. Citado nas páginas 47 e 49.

WEIBEL, C. A. An Introduction to Homological Algebra. Cambridge: Cambridge University Press, 1994. Citado na página 40.

WEIL, A. Basic Number Theory. New York: Springer, 1995. Citado na página 86. 
APÊNDICE

A

GRUPOS ALGEBRICOS

\section{A.1 Definições básicas}

Definição 73. Um grupo afim $G$ sobre um corpo $K$ é um objeto na categoria de functores representáveis da categoria $\mathrm{K}-\mathrm{Alg} \longrightarrow$ Set.

Se $G$ é representado por uma $K$ - álgebra finitamente gerada, então $G$ é dito de grupo algébrico afim.

Observação 39. Na definição anterior quer dizer que um grupo algébrico afim $G$, não é mais que um functor $G: \mathrm{K}-\mathrm{Alg} \longrightarrow$ Set o qual é isomorfo ao functor $\operatorname{Hom}_{\mathrm{K}-\mathrm{Alg}}(\mathscr{O}(G),-): \mathrm{K}-\mathrm{Alg} \longrightarrow \mathrm{Set}$ , para alguma $K$-álgebra finitamente gerada $\mathscr{O}(G)$ a qual é dita de anel coordenado de $G$. Além disso, existe uma transformação natural $m: G \times G \longrightarrow G$ tais que para cada $K$-álgebra $R$ o morfismo multiplicação

$$
m(R): G(R) \times G(R) \longrightarrow G(R)
$$

da uma estrutura de grupo sobre $G(R), G(R)$ é chamado o grupo de $R$-pontos

Definição 74. Um morfismo de $K$-grupos afim $G \longrightarrow H$ é só uma transformação natural de functores.

O produto de $K$-grupos afins $G$ e $H$,denotado por $G \times H$, é só o functor

$$
\begin{aligned}
: \mathrm{K}-\mathrm{Alg} & \longrightarrow \text { Set } \\
R & \longmapsto G(R) \times H(R)
\end{aligned}
$$

o qual é um functor representado por $\mathscr{O}(G) \otimes_{R} \mathscr{O}(H)$, isto é,

$$
\operatorname{Hom}_{\mathrm{K}-\mathrm{Alg}}(\mathscr{O}(G), R) \times \operatorname{Hom}_{\mathrm{K}-\mathrm{Alg}}(\mathscr{O}(H), R) \cong \operatorname{Hom}_{\mathrm{K}-\mathrm{Alg}}\left(\mathscr{O}(G) \otimes_{R} \mathscr{O}(H), R\right)
$$


Exemplo 15. Seja $G L_{n}$ o functor definido por

$$
\begin{aligned}
G L_{n, K}: \mathrm{K}-\mathrm{Alg} & \longrightarrow \text { Set } \\
R & \longmapsto G L_{n}(R)
\end{aligned}
$$

onde $G L_{n, K}(R)$ é o conjunto das $n \times n$ matrizes invertíveis sobre $R$., isto é, as matrizes com determinante em $R^{\times}$. Claramente $G L_{n, K}$ é um $K$-grupo algébrico afim, desde que é representado pela $K$-álgebra

$$
\frac{K\left[X_{11}, X_{12}, \cdots, X_{n n}, Y\right]}{\left\langle\operatorname{det}\left(X_{i j}\right) Y-1\right\rangle}
$$

onde $\operatorname{det}\left(X_{i j}\right)$ é o polinômio de $n^{2}$ variáveis $X_{11}, X_{12}, \cdots, X_{n n}$ dado por

$$
\operatorname{det}\left(X_{i j}\right):=\sum_{\sigma \in S_{n}} \operatorname{Sgn}(\sigma) X_{1 \sigma(1)} \cdots X_{n \sigma(n)}
$$

o grupo $G L_{1, K}$ é usualmente denotado por $G_{m}$ (grupo multiplicativo), desde que $G_{m}(R)$ pode ser identificado com $R^{\times}$.

Exemplo 16. Seja $S L_{n, K}$ o functor definido por

$$
\begin{aligned}
S L_{n, K}: \mathrm{K}-\mathrm{Alg} & \longrightarrow \text { Set } \\
R & \longmapsto S L_{n}(R)
\end{aligned}
$$

onde $S L_{n, K}(R)$ é o conjunto das $n \times n$ matrizes invertíveis sobre $R$ e determinante 1 . Claramente $S L_{n, K}$ é um $K$-grupo algébrico afim, desde que é representado pela $K$-álgebra

$$
\frac{K\left[X_{11}, X_{12}, \cdots, X_{n n}\right]}{\left\langle\operatorname{det}\left(X_{i j}\right)-1\right\rangle}
$$

Dizemos que $H$ é um subgrupo afim de $G$, se $H$ é um subfunctor fechado de $G$ tais que $H(R)$ é um subgrupo de $G(R)$ para toda $K$-álgebra $R$. O fato que $H$ é um subfunctor de $G$ diz que $H$ é representado por um quociente de $\mathscr{O}(G)$.

\section{A.2 Extensão e restrição de escalares}

Definição 75. Sejam $L$ uma $K$-álgebra e $G$ uma $K$-grupo algébrico afim, podemos obter um $L$ grupo algébrico afim $G_{L}$ o qual é dito de extensão de escalar. Suponhamos $G=\operatorname{Hom}(\mathscr{O}(G),-)$ nos definimos

$$
\begin{aligned}
G_{L}: \mathrm{L}-\mathrm{Alg} & \longrightarrow \text { Set } \\
R & \longmapsto \operatorname{Hom}_{\mathrm{L}-\mathrm{Alg}}\left(\mathscr{O}(G) \otimes_{K} L, R\right)
\end{aligned}
$$


Definição 76. Seja $G$ um $L$-grupo algébrico afim, podemos definir um $K$-grupo algébrico afim denotado por $\operatorname{Res}_{L / K} G$ dito de restrição por escalares definido por

$$
\begin{aligned}
\operatorname{Res}_{L / K} G: \mathrm{K}-\mathrm{Alg} & \longrightarrow \text { Set } \\
R & \longmapsto G\left(R \otimes_{K} L\right)
\end{aligned}
$$

Se $\operatorname{Res}_{L / K} G$ é representável e dado por um grupo afim , dizemos que a restrição por escalares existe.

Proposição 35. Seja L uma $K$-álgebra e como $K$-modulo é projetivo finitamente gerado. Então, para cada $L$-grupo afim $G$, existe a restrição por escalares $\operatorname{Res}_{L / K} G$. O functor

$$
H \longmapsto H_{L} \quad \text { e } H \longmapsto \operatorname{Res}_{L / K} H
$$

são adjuntos, isto é, existe uma bijecção natural

$$
\operatorname{Hom}_{L}\left(H_{L}, W\right) \cong \operatorname{Hom}_{K}\left(H, \operatorname{Res}_{L / K} H\right)
$$

Demonstração. Vide [(MILNE, 2012), Capitulo V,5]

Proposição 36. Seja $K^{\prime} / K$ uma extensão finita de corpos e separável, e seja $\bar{K}$ um corpo contendo todas as conjugações de $K^{\prime}$, isto é $\left|\operatorname{Hom}_{K}\left(K^{\prime}, \bar{K}\right)\right|=\left[K^{\prime}: K\right]$ então

$$
\left(\operatorname{Res}_{K^{\prime} / K} G\right)_{K} \cong \prod_{\alpha: K^{\prime} \rightarrow \bar{K}} \alpha G
$$

onde $\alpha G$ é o $K$-grupo afim $G$ obtido pela extensão por escalares com respeito a $\alpha: K^{\prime} \longrightarrow \bar{K}$

Demonstração. Vide [(MILNE, 2012), Capitulo V,5]

Exemplo 17. Sejam $F$ um corpo numérico e $G^{\prime}=S L_{n, F}$ tomando a sua restrição $G:=\operatorname{Res}_{F / \mathbb{Q}} G^{\prime}$, então $G(\mathbb{R})$ é um grupo de Lie Real e se descompõe como $r_{1}$ copias de $S L_{n}(\mathbb{R})$ vezes $r_{2}$ copias de $S L_{n}(\mathbb{C})$

$$
S L_{n}(\mathbb{R}) \times \cdots \times S L_{n}(\mathbb{R}) \times S L_{n}(\mathbb{C}) \times \cdots \times S L_{n}(\mathbb{C})
$$

onde $r_{1}$ é o numero de lugares reais sobre $F$ e $r_{2}$ é o numero de lugares complexos sobre $F$. 

APÊNDICE

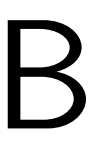

\section{TEORIA DE HOMOTOPIA}

\section{B.1 Homomorfismo de Hurewicz}

Definição 77. Seja $X$ um espaço topológico conexo, dizemos que $X$ é $(n-1)$-conexo se $\pi_{i}(X, x)=0$ para todo $1 \leq i \leq n-1$.

Definição 78. Seja $(X, x)$ um espaço topológico com ponto base. Para cada $n \geq 1$, existe um homomorfismo natural $\mathrm{h}: \pi_{n}(X, x) \longrightarrow H_{n}(X, \mathbb{Z})$ dito de homomorfismo de Hurewicz, definido por

$$
\begin{aligned}
& \mathrm{h}: \pi_{n}(X, x) \longrightarrow H_{n}(X, \mathbb{Z}) \\
& {[f] \longmapsto\left[f_{*}\left(\left[S^{n}\right]\right)\right] }
\end{aligned}
$$

onde $\left[S^{n}\right]$ denota ao gerador do grupo de homologia $H_{n}\left(S^{n}, \mathbb{Z}\right) \cong \mathbb{Z}$.

Teorema 28 (Hurewicz). Seja $X$ um espaço topológico ( $n-1)$-conexo $(n \geq 1)$. Então o homomorfismo de Hurewicz

$$
\begin{aligned}
& \mathrm{h}: \pi_{n}(X, x) \longrightarrow H_{n}(X, \mathbb{Z}) \\
& {[f] \longmapsto\left[f_{*}\left(\left[S^{n}\right]\right)\right] }
\end{aligned}
$$

é um isomorfismo.

Demonstração. Vide [(DIECK, 2008), Teorema 20.1.1]

\section{B.2 Teorema dos coeficientes universais}

Teorema 29 (Teorema dos coeficientes universais para Homologia). Sejam $X$ um espaço topológico, $R$ um DIP (Domínio de ideais principais) e $M$ um $R$-modulo, então existe uma 
sequencia exata curta para todo $n \geq 0$ tais que

$$
0 \longrightarrow H_{n}(X ; R) \otimes_{R} M \stackrel{\alpha}{\longrightarrow} H_{n}(X ; M) \longrightarrow \operatorname{Tor}\left(H_{n-1}(X ; R), M\right) \longrightarrow 0
$$

onde $\alpha:[z] \otimes m \mapsto[z \otimes m]$, a qual escinde, isto é

$$
H_{n}(X ; M)=H_{n}(X ; R) \otimes_{R} M \bigoplus \operatorname{Tor}_{1}^{R}\left(H_{n-1}(X) ; M\right)
$$

para cada $n \geq 0$. Mais,a sequencia não escinde de maneira natural.

Demonstração. Vide [(MAY, 1999), Capitulo 17.1]. Ver [J.P. May, A concise course in Algebraic Topology, Teorema (Universal coefficient), pag 132].

Teorema 30 (Teorema dos coeficientes universais para Cohomologia). Sejam $X$ um espaço topológico, $R$ um DIP (Domínio de ideais principais) e $M$ um $R$-modulo, então existe uma sequencia exata curta para todo $n \geq 0$ tais que

$$
0 \longrightarrow \operatorname{Ext}_{R}^{1}\left(H_{n-1}(X), M\right) \longrightarrow H^{n}(X ; M) \stackrel{\beta}{\longrightarrow} \operatorname{Hom}\left(H_{n}(X ; R), M\right) \longrightarrow 0
$$

onde $\beta([f])([z])=f(z)$ onde $[f] \in H^{n}(X ; M)$ e $[z] \in H_{n}(X ; R)$, a qual escinde, isto é

$$
H^{n}(X ; M)=\operatorname{Hom}\left(H^{n}(X ; R), M\right) \bigoplus \operatorname{Ext}_{R}^{1}\left(H_{n-1}(X), M\right)
$$

para cada $n \geq 0$. Mais,a sequencia não escinde de maneira natural.

Demonstração. Vide [(MAY, 1999), Capitulo 17.1].

\section{B.3 Fibrações}

Nesta secção $E, B$ e $Y$ denotaram espaços topológicos, $I=[0,1]$. Denotamos por $i_{0}$ : $Y \longrightarrow Y \times I$ o mapa definido por

$$
\begin{aligned}
i_{0}: Y & \longrightarrow Y \times I \\
y & \longmapsto(y, 0)
\end{aligned}
$$

Definição 79. Um mapa $p: E \longrightarrow B$ é dito de fibração, se dado um mapa $f: Y \longrightarrow E$ e $h: Y \times I \longrightarrow B$ tais que o seguinte diagrama

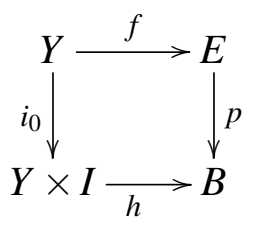

comuta, existe um mapa $H: Y \times I \longrightarrow E$ tais que $p \circ H=h$. 
Denotamos por $F \hookrightarrow E \stackrel{p}{\longrightarrow} B$ à fibração $E \stackrel{p}{\longrightarrow} B$ com fibra $F \cong p^{-1}(b)$, onde $b$ é um ponto base de $B$.

Proposição 37. Seja $p: E \longrightarrow B$ uma fibração, seja $b \in B$ um ponto base de $B$ e seja $F:=p^{-1}(b)$ uma fibra. Então existe uma sequência exata longa relacionando os grupos de homotopia de $E$, $B, F$, isto é,

$$
\cdots \rightarrow \pi_{n}(F) \stackrel{i_{*}}{\rightarrow} \pi_{n}(E) \stackrel{p_{*}}{\rightarrow} \pi_{n}(B) \stackrel{\partial}{\rightarrow} \pi_{n-1}(F) \rightarrow \cdots \rightarrow \pi_{0}(F) \rightarrow \pi_{0}(E) \rightarrow \pi_{0}(B)
$$

Demonstração. Vide [(DIECK, 2008), Teorema 6.3.2]

\section{B.4 Sequência espectral de Serre-Leray}

Definição 80. Uma família de objetos $E_{p, q}^{r}$ (onde $E_{p, q}^{r}=0$, a menos que $p, q \geq 0$ ) munida de umas diferenciais $d_{p, q}^{r}: E_{p, q}^{r} \longrightarrow E_{p-r, q+r-1}^{r}$ é dita de sequência espectral homológica se, $d_{p, q}^{r} \circ d_{p+r, q-r+1}^{r}=0$ e o objeto $E_{p, q}^{r+1}$ é isomorfo à homologia do complexo $E_{*, *}^{r}$ no nível $\{p, q\}$, isto é

$$
\cdots \longrightarrow E_{p+r, q-r+1}^{r} \stackrel{d_{p+r, q-r+1}^{r}}{\longrightarrow} E_{p, q}^{r} \stackrel{d_{p, q}^{r}}{\longrightarrow} E_{p-r, q+r-1}^{r} \longrightarrow \cdots
$$

e

$$
E_{p, q}^{r+1} \cong \frac{\operatorname{ker}\left(d_{p, q}^{r}\right)}{\operatorname{Im}\left(d_{p+r, q-r+1}^{r}\right)}
$$

Dualmente, podemos definir uma sequência espectral cohomologica.

Definição 81. Uma família de objetos $E_{r}^{p, q}$ (onde $E_{r}^{p, q}=0$, a menos que $p, q \geq 0$ ) munida de umas diferenciais $d_{r}^{p, q}: E_{r}^{p, q} \longrightarrow E_{r}^{p+r, q-r+1}$ é dita de sequência espectral cohomológica se, $d_{r}^{p, q} \circ d_{r}^{p+r, q-r+1}=0$ e o objeto $E_{r+1}^{p, q}$ é isomorfo à cohomologia do complexo $E_{r}^{*, *}$ no nível $\{p, q\}$, isto é

$$
\cdots \longrightarrow E_{r}^{p-r, q+r-1} \stackrel{d_{r}^{p-r, q+r-1}}{\longrightarrow} E_{r}^{p, q} \stackrel{d_{r}^{p, q}}{\longrightarrow} E_{r}^{p+r, q-r+1} \longrightarrow \cdots
$$

e

$$
E_{r+1}^{p, q} \cong \frac{\operatorname{ker}\left(d_{r}^{p, q}\right)}{\operatorname{Im}\left(d_{r}^{p-r, q+r-1}\right)} .
$$

Teorema 31 (A sequência espectral homológica Leray-Serre). Seja $G$ um grupo abeliano. Dada uma fibração $F \hookrightarrow E \stackrel{p}{\longrightarrow} B$, onde $B$ é conexo por caminhos, $F$ é conexo. Então existe uma sequência espectral, $\left\{E_{*, *}^{r}, d^{r}\right\}$, convergindo a $H_{*}(E, G)$, com

$$
E_{p, q}^{2} \cong H_{p}\left(B ; \mathscr{H}_{q}(F, G)\right)
$$

a homologia do espaço $B$ com coeficientes locais na homologia da fibra de $p$. Além disso, esta sequencia espectral é natural com respeito aos mapas entre fibrações preservando fibra. 
Teorema 32 (A sequência espectral cohomológica Leray-Serre). Seja $R$ um anel comutativo com unidade. Dada uma fibração $F \hookrightarrow E \stackrel{p}{\longrightarrow} B$, onde $B$ é conexo por caminhos, $F$ é conexo. Então existe uma sequência espectral de álgebras $\left\{E_{r}^{*, *}, d_{r}\right\}$, convergindo a $H^{*}(E, R)$ como uma álgebra, com

$$
E_{2}^{p, q} \cong H^{p}\left(B ; \mathscr{H}^{q}(F, G)\right)
$$

a cohomologia do espaço $B$ com coeficientes locais na fibra da cohomologia de $p$. Esta sequência espectral é natural com respeito aos mapas entre fibrações preservando fibra. Além disso, o produto cup $\smile$ sobre a cohomologia com coeficientes locais e o produto.$_{2}$ sobre $E_{2}^{* * *}$ são relacionados por

$$
u \cdot 2 v=(-1)^{p^{\prime} q} u \smile v
$$

onde $u \in E_{2}^{p, q}$ e $v \in E_{2}^{p^{\prime}, q^{\prime}}$.

Exemplo 18. O grupo de Lie clássico $S U(n)$ é o grupo de $n \times n$ matrizes unitárias $\left(A \bar{A}^{t}=I\right)$ de determinante 1 . fixando um vector $v_{0} \in \mathbb{C}^{n}$, definimos

$$
\begin{aligned}
p: S U(n) & \longrightarrow S^{2 n-1} \\
A & \longmapsto A v_{0} .
\end{aligned}
$$

pode-se mostrar que este mapa é uma fibração com fibra o subgrupo de $S U(n)$ que fixa $v_{0}$, o qual é $S U(n-1)$. O subgrupo $S U(2)$ pode ser identificado com a esfera $S^{3}$. E assim

$$
H^{*}(S U(2) ; R) \cong H^{*}\left(S^{3} ; R\right) \cong \Lambda\left(x_{3}\right)
$$

Considerando a fibração $S U(n-1) \hookrightarrow S U(n) \stackrel{p}{\longrightarrow} S^{2 n-1}$ e aplicando a sequencia espectral de Leray-Serre. Suponhamos que $x_{2 n-1}$ gera $H^{*}\left(S^{2 n-1} ; R\right)$ como uma álgebra exterior, isto é , $H^{*}\left(S^{2 n-1} ; R\right) \cong \Lambda\left(x_{2 n-1}\right)$. Por indução, nos temos

$$
H^{*}(S U(n-1) ; R) \cong \Lambda\left(x_{3}, x_{5}, \cdots, x_{2 n-3}\right)
$$

Desde que esta é uma sequencia espectral de álgebras, nos precisamos só considerar os geradores da álgebra para descrever as diferenciais. Para $n \geq 2, S^{2 n-1}$ é simplesmente conexo e assim o sistema de coeficientes locais sobre o espaço base é simples. Além disso, pelo teorema dos coeficientes universais, temos

$$
E_{2}^{*, *} \cong H^{*}(B ; R) \otimes H^{*}(F ; R) \cong \Lambda\left(x_{2 n-1}\right) \otimes \Lambda\left(x_{3}, x_{5}, \cdots, x_{2 n-3}\right) .
$$

Os geradores da álgebra são encontrados em bigraus, de modo que todas as diferencias são zero e a sequencia espectral colapsa no termo $E_{2}$. E assim, temos

$$
H^{*}(S U(n) ; R) \cong \Lambda\left(x_{3}, x_{5}, \cdots, x_{2 n-1}\right)
$$

e a indução é completada. 
Exemplo 19. Do exemplo anterior, podemos estender para calcular $H^{*}(U(n) ; R)$, onde $U(n)$ é o grupo de transformações lineares de $\mathbb{C}^{n}$ que preservam o produto interno. O grupo $U(n)$ se relaciona a $S U(n)$ via a fibração:

$$
S U(n) \hookrightarrow U(n) \stackrel{\operatorname{det}}{\longrightarrow} S^{1}
$$

e além disso, $U(n) \simeq U(1) \times S U(n) \simeq S^{1} \times S U(n)$. Assim, pelo teorema dos coeficientes universais temos

$$
H^{*}(U(n) ; R) \cong H^{*}\left(S^{1} ; R\right) \otimes H^{*}(S U(n) ; R) \cong \Lambda\left(x_{1}, x_{3}, \cdots, x_{2 n-1}\right)
$$




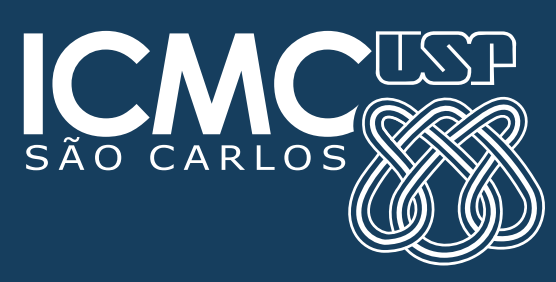

